\title{
Superdispersão em dados binomiais hierárquicos
}

\author{
Lílian Nati
}

\author{
TESE APRESENTADA \\ $\mathrm{AO}$ \\ Instituto DE MATEMÁtica E EstatísticA \\ DA \\ UNIVERSIDADE DE SÃO PAULO \\ PARA \\ OBTENÇÃO DO TÍTULO \\ $\mathrm{DE}$ \\ DouTOR EM Cî̂NCIAS
}

\author{
Área de Concentração: Estatística \\ Orientador: Prof. Dr. Dalton Francisco de Andrade
}





\title{
Superdispersão em dados binomiais hierárquicos
}

\author{
Este exemplar corresponde à redação \\ final da tese devidamente corrigida \\ e defendida por Lílian Nati \\ e aprovada pela Comissão Julgadora.
}

Banca Examinadora:

- Prof. Dalton Francisco de Andrade (orientador) - UFSC

- Prof. Dr. Dr. Julio da Motta Singer - IME-USP

- Profa. Dra. Clarice Garcia Borges Demétrio - ESALQ-USP

- Prof. Dr. Helio dos Santos Migon - UFRJ

- Prof. Dr. Gauss Moutinho Cordeiro - UFRPE 



\section{A Pedra}

"O distraído nela tropeçou.

O bruto a usou como projétil.

O empreendedor, usando-a, construiu.

O camponês, cansado da lida, dela fez assento.

Para meninos, foi brinquedo.

Drummond a poetizou.

Já David matou Golias e Michelangelo extraiu-lhe a mais bela escultura.

E em todos esses casos, a diferença não esteve na pedra, mas no homem!"

(autor desconhecido)

Aos meus pais Sérgio e Alcione, a minha irmã Élen, e ao meu marido Frederico. 



\section{Agradecimentos}

É com imensa satisfação que declaro a todos que me acompanharam nesta longa trajetória: MISSÃO CUMPRIDA!

Agradeço primeiramente a Deus, por sempre ter iluminado meu caminho com saúde, vivacidade e pessoas de grande valor, que me ofereceram seu apoio com entusiasmo, inspiração, generosidade e competência.

Aos meus pais Sérgio e Alcione, sempre muito conscientes da importância dos estudos, meu eterno muito obrigada pela família estruturada que me proporcionaram, cheia de amor, carinho e dedicação. Vocês são 100\% responsáveis por eu ter chegado tão longe!

Agradeço a minha irmã Élen, que com seu espírito de luta e capacidade de enfrentar desafios, estimulou-me a vencer minhas apreensões.

Ao meu orientador Prof. Dalton Andrade, que com paciência esperou pela conclusão deste trabalho, sou grata pelo apoio, confiança e compreensão, em especial nos momentos difíceis.

Ao Prof. Julio Singer, que com imensa boa vontade esteve sempre pronto a me receber, contribuindo com orientações, sugestões, correções e, também, com sua amizade. Seu incentivo foi fundamental para a evolução desta tese.

Obrigada ao Prof. Gauss Cordeiro, à Profa. Clarice Demétrio e ao Prof. Helio Migon que, juntamente com o Prof. Julio Singer e o Prof. Dalton Andrade, concederam-me a honra de ter em minha banca examinadora profissionais tão competentes e respeitáveis, que muito acrescentaram 
com suas idéias e sugestões.

Não posso deixar de agradecer aos funcionários do Serviço de Alunos de Pós-Graduação do IME, em especial a Sebastião Pinho, Rose Lima e Alessandra Bernardo, que sempre me atenderam prontamente, com muita paciência e simpatia.

Agradeço ao meu querido amigo David Pires pelo inestimável auxílio, espontâneo e voluntário. É admirável a sua capacidade de fazer o bem, de estender a sua mão até mesmo a um desconhecido (para quem não sabe, nosso primeiro contato ocorreu por e-mail). Foi preciosa a sua colaboração com o meu gigante trabalho computacional, concluído em tempo recorde.

Ao Sr. Francisco Ribacionka, da equipe do Laboratório de Computação Científica Avançada (LCCA), agradeço imensamente pela atenção e ajuda com o uso do Cluster Linux Alcatéia. Além de excelente profissional, foi de extrema gentileza e fez tudo que esteve ao seu alcance para que eu concluísse a parte computacional de minha tese.

Agradeço ao Prof. Siang Wun Song, que muito gentilmente permitiu o meu acesso ao Cluster IBM, concedido pela FAPESP (Proc. No. 04/08928-3) ao seu grupo de estudo no Departamento de Ciência da Computação do IME. Sem essa poderosa máquina teria sido impossível terminar esse trabalho.

É com muito prazer que compartilho essa vitória com estas mulheres competentes que tanto me incentivaram: Luciana Jensen, Evelyn Cremonese e Helena Toscano. Sem vocês eu não estaria aqui escrevendo esta página!

Dedico esta conquista em especial ao meu admirável marido, Frederico Poleto, que acompanhou ativamente o desenvolvimento deste trabalho, ajudando-me incansavelmente, principalmente no gerenciamento das minhas intermináveis simulações! Obrigada por todo o suporte afetivo e profissional, que me encorajou, impulsionou-me e não me deixou desistir de realizar esse sonho. Você é uma inspiração, um exemplo de determinação e competência e um ser humano ímpar!

Aos meus sogros, Sérgio e Dagmar, sou muito grata pela forma carinhosa como me acolheram, sempre me encorajando e torcendo por mim.

A D. Aurora, minha avó Nilcéa e D. Zezinha, manifesto o meu apreço por todas as vibrações 
positivas e preces. Obrigada a minha madrinha e avó Maria, que com certeza deve olhar por mim de onde ela está.

Agradeço aos meus amigos de longa data, que sabem bem ou sentiram na pele o que é fazer um doutorado no IME: Carine Savalli, Fernando Redígolo, André Samartini, Liliam Lima, Mariana Cúri, Ângela Paes, Maria Paula Chicarino, Raquel Valle e Luís Gustavo Esteves. É extraordinário o quanto servimos de apoio uns aos outros durante essa jornada insana e, também, o quanto esta fortaleceu nossos laços de amizade e companheirismo!

Obrigada aos meus demais amigos, Alexandra Calabrez, Ricardo Raitz, Soraia Teijeiro, Kátia Fontana, Helio Arizono, Paulo de Tarso Rosa, Cesar Franco, Rodrigo Tavares, Ricardo Fernandes, Rovane Machado, Cintia Burket e Rejane Figueiredo (perdão se deixei de citar alguém no calor deste momento), que juntamente com os amigos anteriormente mencionados, sempre tornaram minha vida mais agradável e divertida. Temos tantas histórias boas e engraçadas pra contar! E o melhor é que continuamos a fazer histórias, com cada vez mais participantes (filhinhos)...

Obrigada ao meu amigo Prof. José Oscar Bustillos, pelos conselhos e sábias palavras de incentivo, pela convivência tão agradável e pela alegria que transmite a todos ao seu redor.

E pra quem conseguiu ler até aqui e já deve estar dizendo: "ela só faltou agradecer aos seus cachorrinhos", é verdade! Obrigada ao meu cãozinho querido Pitty e minhas cachorrinhas Meg e a Ully, por todos os afagos, amor e diversão! Faltou mencionar o gatinho que mais parece um cachorro: valeu Milk!

Agora sim, posso comemorar meu título de doutora tão suado! Fiquem com Deus. 



\section{Resumo}

Para analisar dados binários oriundos de uma estrutura hierárquica com dois níveis (por exemplo, aluno e escola), uma alternativa bastante utilizada é a suposição da distribuição binomial para as unidades experimentais do primeiro nível (aluno) condicionalmente a um efeito aleatório proveniente de uma distribuição normal para as unidades do segundo nível (escola). Neste trabalho, propõe-se a adição de um efeito aleatório normal no primeiro nível de um modelo linear generalizado hierárquico binomial para contemplar uma possível variabilidade extra-binomial decorrente da dependência entre os ensaios de Bernoulli de um mesmo indivíduo. Obtém-se o processo de estimação por máxima verossimilhança para este modelo a partir da verossimilhança marginal dos dados, após uma dupla aplicação do método de quadratura de Gauss-Hermite adaptativa como aproximação para as integrais dos efeitos aleatórios. Realiza-se um estudo de simulação para contrastar propriedades inferenciais do modelo aspirante com o modelo linear generalizado binomial, um modelo de quase-verossimilhança e o tradicional modelo linear generalizado hierárquico em dois níveis.

Palavras-chave: modelos hierárquicos, modelos multiníveis, modelos lineares generalizados mistos, dados binomiais, superdispersão. 



\section{Abstract}

A common alternative when analyzing binary data originated from a two-level hierarchical structure (for instance, student and school) is to assume a binomial distribution for the experimental units of the first level (student) conditionally to a normal random effect for the second level units (school). In this work, we propose the inclusion of a second normal random effect in the first level to contemplate a possible extra-binomial variability due to the dependence among the Bernoulli trials in the same individual. We obtain the maximum likelihood estimation process for this hierarchical model starting from the marginal likelihood of the data, after a double application of the adaptive Gauss-Hermite quadrature as an approximation of the integrals of the random effects. We conduct a simulation study to compare the inferential properties of the advocated model with the generalized linear (binomial) model, a quasi-likelihood model and the usual two-level hierarchical generalized linear model.

Keywords: hierarchical models, multilevel models, generalized linear mixed models, binomial data, overdispersion. 



\section{Sumário}

Resumo

Abstract $\quad$ xiii

Lista de abreviaturas $\quad$ xvii

1 Aspectos gerais e motivação 1

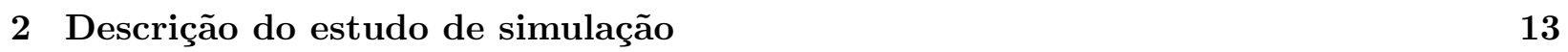

2.1 Geração de dados . . . . . . . . . . . . . . . . . . . . . . . . . 13

2.2 Delineamento do estudo de simulação . . . . . . . . . . . . . . . . . 14

2.3 Método de quadratura de Gauss-Hermite adaptativa . . . . . . . . . . . . . 17

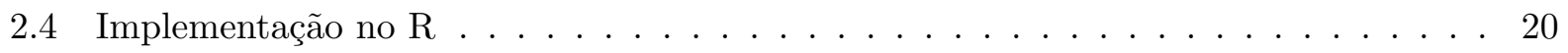

3 Resultados $\quad 23$

3.1 Vieses dos estimadores dos componentes de variância $\left(\tau_{0}\right.$ e $\left.\sigma\right) \ldots \ldots$. . . . . 24 


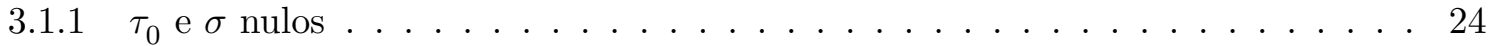

3.1 .2 Somente $\tau_{0}$ não nulo . . . . . . . . . . . . . . . . 24

3.1 .3 Somente $\sigma$ não nulo . . . . . . . . . . . . . . . . . . . 25

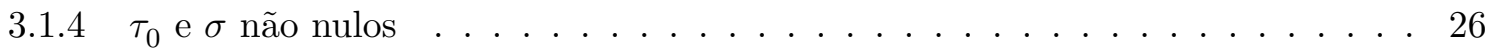

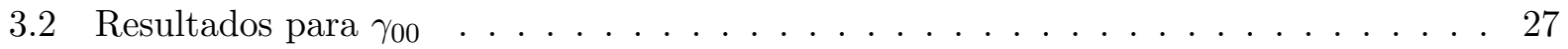

3.2 .1 Avaliação do estimador $\hat{\gamma}_{00} \ldots \ldots \ldots \ldots \ldots \ldots \ldots \ldots$

3.2 .2 Avaliação da taxa de rejeição para $\gamma_{00} \ldots \ldots \ldots \ldots$. . . . . . . . . . 31

4 Aplicação $\quad 35$

5 Conclusões $\quad 41$

A Esperanças e covariâncias marginais $\quad 45$

A.1 Cálculo de $\mathrm{E}\left[p_{i j}\right], \operatorname{Var}\left[p_{i j}\right]$ e $\operatorname{Cov}\left[p_{i j}, p_{i^{\prime} j}\right] \ldots \ldots \ldots \ldots \ldots$

A.2 Cálculo de $\mathrm{E}\left[Y_{i j}\right], \operatorname{Var}\left[Y_{i j}\right]$ e $\operatorname{Cov}\left[Y_{i j}, Y_{i^{\prime} j}\right] \ldots \ldots \ldots \ldots \ldots$

B Tabelas $\quad 49$

$\begin{array}{ll}\text { C Gráficos referentes a } \tau_{0} \text { e } \sigma & 61\end{array}$

$\begin{array}{ll}\text { D Gráficos referentes a } \gamma_{00} & 71\end{array}$

Referências Bibliográficas $\quad 85$ 


\title{
Lista de abreviaturas
}

\author{
a.a. amostra aleatória \\ DE diretoria de ensino \\ DP desvio padrão \\ EP erro padrão \\ f.d.p. função densidade de probabilidade \\ GL grau(s) de liberdade \\ IC intervalo de confiança \\ i.i.d. independentes e identicamente distribuídos \\ MV máxima verossimilhança \\ MCMC Markov Chain Monte Carlo \\ MLG modelo linear generalizado \\ MLH modelo linear hierárquico \\ MLGH modelo linear generalizado hierárquico \\ MLGM modelo linear generalizado misto \\ QV quase-verossimilhança \\ QVP quase-verossimilhança penalizada \\ QGHA quadratura de Gauss-Hermite adaptativa \\ QGR ajuste do modelo 2, usando a QGHA disponível no pacote glmmML do R \\ QG2 ajuste do modelo 2, usando a QGHA implementada no programa desenvolvido no R \\ QG3 ajuste do modelo 3, usando a QGHA implementada no programa desenvolvido no R
}





\section{Capítulo 1}

\section{Aspectos gerais e motivação}

Estruturas hierárquicas de dados, encontradas com freqüência em diversos estudos, são caracterizadas pela presença de unidades de observação que constituem um agrupamento de outras unidades de diferente natureza. Estas últimas podem, também, compor agrupamentos de outras unidades de interesse, estabelecendo-se diferentes níveis de hierarquia de unidades amostrais. Os modelos que descrevem este tipo de estrutura são conhecidos como modelos hierárquicos ou modelos multiníveis.

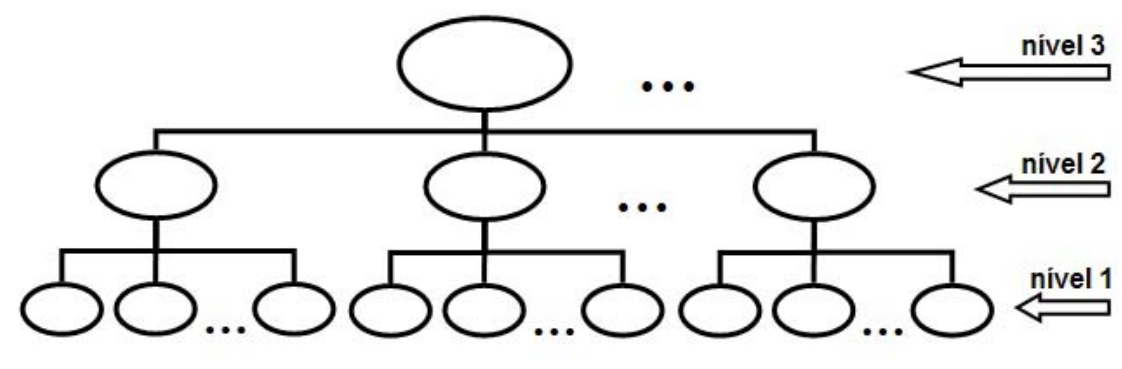

Figura 1.1: Estrutura hierárquica em 3 níveis

Quando se assume que a variável resposta em um modelo multinível tem distribuição normal, pode-se utilizar a teoria dos modelos lineares hierárquicos (MLHs) ou modelos lineares multiníveis (veja Raudenbush e Bryk (2002), Natis (2000) ou Goldstein (2003), por exemplo). Incluindo-se variáveis explicativas naturalmente associadas a cada nível de hierarquia, busca-se explicar a variabilidade apresentada entre as unidades do nível em questão. Caso não seja possível explicá-la 
totalmente, os MLHs permitem a incorporação de efeitos aleatórios, supostos normalmente distribuídos.

Na formulação geral do MLH em 2 níveis descrita em Raudenbush e Bryk (2002) tem-se:

$$
\text { modelo de nível 1: } \quad Y_{i j}=\beta_{0 j}+\beta_{1 j} X_{1 i j}+\beta_{2 j} X_{2 i j}+\cdots+\beta_{Q j} X_{Q i j}+r_{i j}
$$

$\mathrm{e}$

$$
\text { modelo de nível 2: } \quad \beta_{q j}=\gamma_{q 0}+\sum_{s=1}^{S_{q}} \gamma_{q s} W_{q s j}+\varepsilon_{q j}
$$

em que $i=1,2, \ldots, n_{j}$ e $j=1,2, \ldots, J$ identificam as unidades dos níveis 1 e 2 respectivamente e $X_{q i j}$ são as variáveis explicativas do nível $1, \operatorname{com} q=0,1,2, \ldots, Q$ e $X_{0 i j}=1$. Os erros aleatórios $r_{i j}$ (erros do nível 1) são independentes e normalmente distribuídos com média zero e variância $\phi$. Caso as unidades do nível 2 não apresentem o mesmo coeficiente $\beta_{q j}$, busca-se explicar tais diferenças pela inclusão das variáveis $W_{q s j}, s=1,2, \ldots, S_{q}$. Se ainda houver variabilidade remanescente, pode-se acomodá-la pela introdução dos efeitos aleatórios $\varepsilon_{q j}$, chamados de erros aleatórios do nível 2, sendo $\varepsilon_{q j} \sim N\left(0, \tau_{q}^{2}\right)$ independentes $\forall j \neq j^{\prime}$ e $\operatorname{Cov}\left[\varepsilon_{q j}, \varepsilon_{q^{\prime} j}\right]=\tau_{q q^{\prime}}$. Essa formulação em níveis de hierarquia, motivada pela própria natureza dos dados, proporciona maior facilidade na descrição, interpretação e análise das diferentes fontes de variação.

Pode-se subdividir os modelos de regressão em hierárquicos (multiníveis) e não hierárquicos e, adicionalmente, em gaussianos e não gaussianos. O termo hierárquico aparece na literatura em outros contextos, por isso é importante salientar que neste trabalho esta palavra designará apenas modelos multiníveis, entre os quais serão avaliados alguns modelos não gaussianos.

Quando a variável resposta assume alguma distribuição da família exponencial diferente da gaussiana, a variância depende do valor esperado. Neste caso, sem a homocedasticidade requerida pelos MLHs, recorre-se então aos chamados modelos lineares generalizados hierárquicos (MLGHs), que estabelecem uma relação funcional entre a média da variável resposta e um preditor linear e, assim como os MLHs, podem incluir variáveis explicativas e efeitos aleatórios de acordo com o nível de hierarquia a que eles correspondem.

Quando se supõe que os efeitos aleatórios associados aos níveis mais altos da hierarquia (maiores que 1) são normalmente distribuídos, os MLGHs se enquadram na classe dos modelos lineares 
generalizados mistos (MLGMs) (veja Molenberghs e Verbeke (2005)). A literatura apresenta outras propostas em que os efeitos aleatórios podem assumir outras distribuições, como em Lee e Nelder (1996), que propõem distribuições beta e gama para dados binários e de contagem, respectivamente. Para facilitar a identificação do modelo abordado, a sigla MLGH por vezes é acrescida de uma palavra composta referente à distribuição condicional da resposta e à distribuição dos efeitos aleatórios. Por exemplo: a abreviação MLGH binomial-normal corresponde a um MLGH com resposta binomial e efeitos aleatórios normais.

Na formulação geral do MLGH em 2 níveis descrita em Raudenbush e Bryk (2002), os erros aleatórios $r_{i j}$ não figuram na equação do nível 1, já que eles são avaliados por meio dos componentes da função de desviância.

Exemplo 1.1 Considere um estudo em que os dados foram obtidos em uma amostra aleatória (a.a.) de diretorias de ensino (DEs) do Estado de São Paulo, a que estão subordinadas escolas com a $8^{\mathrm{a}}$ série do ensino fundamental. Dentre as diretorias amostradas, selecionou-se uma amostra dessas escolas e em cada uma delas foi selecionada uma amostra de alunos da $8^{\mathrm{a}}$ série. Os alunos, as escolas e as DEs correspondem às unidades de observação dos níveis de hierarquia 1, 2 e 3, respectivamente. Os alunos realizaram uma prova de Língua Portuguesa composta de 20 itens de múltipla escolha, todos com conteúdos desconexos e 4 alternativas. As respostas a cada item da prova foram registradas com o objetivo de avaliar quais características de cada aluno, escola ou DE podem ter influenciado o desempenho, medido pelo número de respostas corretas. As características de cada aluno foram obtidas por meio de um questionário, a partir do qual foram geradas as variáveis independentes do nível 1, como sexo, aspiração principal, número de repetências, tipos de leitura que costuma fazer e auto-avaliação como leitor. Características das escolas também foram coletadas; entre elas destacam-se perfil da postura dos professores, classificação segundo as séries oferecidas, condição dos espaços pedagógicos e adequação do espaço físico. A maioria dessas características foi obtida a partir de um questionário aplicado à direção da escola, dando origem às variáveis independentes do nível 2. Com relação às DEs, a sua localização (capital ou interior) foi utilizada como variável independente do nível 3.

No Exemplo 1.1, a resposta de cada aluno em cada item da prova pode ser considerada como um ensaio de Bernoulli em que o acerto configura o evento de interesse. O número de acertos (escore) de cada aluno pode ser visto como uma medida de seu desempenho na prova. Suponha que 
o escore do aluno (variável resposta) siga uma distribuição binomial com uma certa probabilidade de acerto. A variabilidade do escore talvez seja parcialmente explicada por algumas das variáveis independentes, mas ainda pode restar uma parcela não explicada, seja pela influência de fatores desconhecidos ou que não podem ser controlados ou simplesmente porque existe uma variabilidade de natureza aleatória entre alunos, entre escolas e entre DEs, o que sugere a incorporação de efeitos aleatórios de acordo com os níveis de hierarquia, contemplando a possível correlação entre alunos que estudam na mesma escola e entre escolas que pertencem à mesma DE. Num MLGH com resposta binomial todas estas particularidades podem ser levadas em conta. No entanto, a distribuição do escore, condicionalmente aos efeitos aleatórios e às variáveis explicativas, pode não apresentar a variabilidade natural de um modelo binomial, devido à possível dependência entre as respostas de um mesmo aluno e, também, a possíveis diferenças entre as probabilidades de acerto dos itens. Trata-se de um provável caso de superdispersão ${ }^{1}$, que inspira propor uma forma adequada de explicá-la.

A seguir, serão ilustrados diferentes modelos, para ajustar a dados de uma estrutura hierárquica em apenas 2 níveis, aluno e escola, de modo a facilitar a explanação do que será desenvolvido neste trabalho.

Considere uma a.a. de $J$ escolas em cada uma das quais $n_{j}$ alunos foram selecionados. Seja $Y_{i j}$ o escore que o $i$-ésimo aluno da $j$-ésima escola obteve em uma prova com $K$ itens, em cada um dos quais a probabilidade de acerto é assumida como

$$
p_{i j}=\operatorname{Prob}[\text { acerto } \mid \text { aluno } i \text {, escola } j] \quad \forall k,
$$

em que $i=1,2, \ldots, n_{j}, j=1,2, \ldots, J$ e $k=1,2, \ldots, K$.

Seja $g\left(p_{i j}\right)$ uma função de ligação, a ser modelada por um preditor linear $\eta_{i j}$. A ligação canônica para a distribuição binomial é

$$
\eta_{i j}=\log \left(\frac{p_{i j}}{1-p_{i j}}\right)
$$

\footnotetext{
${ }^{1}$ Como a subdispersão é um fenômeno menos comum na prática segundo McCullagh e Nelder (1989) e a inclusão de efeitos aleatórios em modelos hierárquicos só admite o aumento da variabilidade, será usado apenas o termo superdispersão.
} 
Será apresentada a seguir uma seqüência de modelos que, por simplicidade, não incluirão variáveis explicativas. Considere em todos os modelos que

$$
Y_{i j} \mid p_{i j} \sim \operatorname{binomial}\left(K, p_{i j}\right), \text { condicionalmente independentes } \forall i \text { e } \forall j \text {. }
$$

Proponha-se agora o seguinte modelo:

\section{Modelo 1.}

e

$$
\text { Nível 1: } \quad \log \left(\frac{p_{i j}}{1-p_{i j}}\right)=\beta_{0 j}
$$

$$
\text { Nível 2: } \quad \beta_{0 j}=\gamma_{00}, \gamma_{00} \text { fixo . }
$$

Este modelo poderia ser ajustado de acordo com a teoria usual para os MLGs (veja McCullagh e Nelder (1989)).

Como $p_{i j}$ é constante, a esperança e a variância condicionais de $Y_{i j}$ coincidem com as respectivas esperança e variância marginais:

$$
\mathrm{E}\left[Y_{i j} \mid p_{i j}\right]=K p_{i j}=K\left(1+e^{-\gamma_{00}}\right)^{-1}=\mathrm{E}\left[Y_{i j}\right]
$$

e

$$
\operatorname{Var}\left[Y_{i j} \mid p_{i j}\right]=K p_{i j}\left(1-p_{i j}\right)=K e^{-\gamma_{00}}\left(1+e^{-\gamma_{00}}\right)^{-2}=\operatorname{Var}\left[Y_{i j}\right]
$$

Esse modelo pode não ser apropriado, pois

1a. a probabilidade de acerto num determinado item pode não ser a mesma para todos os alunos;

1b. pode haver dependência entre as respostas de alunos da mesma escola;

1c. os ensaios de Bernoulli associados ao mesmo aluno podem não ser independentes;

1d. os ensaios de Bernoulli associados ao mesmo aluno talvez não sejam identicamente distribuídos, já que os itens podem ter diferentes probabilidades de acerto. 
A inclusão de covariáveis no primeiro nível do modelo poderia ajudar a explicar as diferenças entre as probabilidades de acerto para alunos da mesma escola. A inclusão de covariáveis no segundo nível do modelo poderia ajudar a explicar as diferenças entre alunos de diferentes escolas, ainda que estes apresentassem os mesmos valores nas covariáveis de nível 1. Mesmo assim poderia haver superdispersão, pois apenas o problema 1a estaria contemplado.

A superdispersão poderia ser detectada por testes de adequação do modelo ou técnicas de diagnóstico para MLGs (veja Paula (2004), por exemplo). Uma das práticas mais utilizadas para tentar acomodar a superdispersão seria supor que a variância continua a ter uma relação com a média como a existente no caso binomial, porém inflacionada por uma constante multiplicativa $\phi$, de modo que as expressões (1.5) a (1.7) permanecem válidas, mas a variância condicional de $Y_{i j}$ passa a ser:

$$
\operatorname{Var}\left[Y_{i j} \mid p_{i j}\right]=\phi K p_{i j}\left(1-p_{i j}\right)=\phi K e^{-\gamma_{00}}\left(1+e^{-\gamma_{00}}\right)^{-2}=\operatorname{Var}\left[Y_{i j}\right]
$$

Aqui $\phi>0$ representa o parâmetro de superdispersão, para o qual existem vários estimadores propostos na literatura (veja Paula (2004), por exemplo). Nota-se que se $\phi=1,(1.8)$ e (1.9) são iguais.

Para tentar explicar a variação extra-binomial, considere o seguinte modelo:

\section{Modelo 2.}

$$
\text { Nível 1: } \quad \log \left(\frac{p_{i j}}{1-p_{i j}}\right)=\beta_{0 j}
$$

e

$$
\begin{aligned}
& \text { Nível } 2: \quad \beta_{0 j}=\gamma_{00}+\varepsilon_{0 j}, \\
& \gamma_{00} \text { fixo e } \varepsilon_{0 j} \sim N\left(0, \tau_{0}^{2}\right) \text { i.i.d. }
\end{aligned}
$$

As expressões da esperança e variância condicionais de $Y_{i j}$ são

$$
\mathrm{E}\left[Y_{i j} \mid p_{i j}\right]=K p_{i j}
$$


$\mathrm{e}$

$$
\operatorname{Var}\left[Y_{i j} \mid p_{i j}\right]=K p_{i j}\left(1-p_{i j}\right)
$$

A média e variância de $p_{i j}$ são expressas, respectivamente, por

$$
\mathrm{E}\left[p_{i j}\right] \cong\left(1+e^{-\gamma_{00}}\right)^{-1}
$$

$\mathrm{e}$

$$
\operatorname{Var}\left[p_{i j}\right] \cong\left[\tau_{0} e^{-\gamma_{00}}\left(1+e^{-\gamma_{00}}\right)^{-2}\right]^{2}
$$

Assim, a esperança e a variância marginais de $Y_{i j}$ são

$$
\mathrm{E}\left[Y_{i j}\right] \cong K\left(1+e^{-\gamma_{00}}\right)^{-1}
$$

$\mathrm{e}$

$$
\operatorname{Var}\left[Y_{i j}\right] \cong K e^{-\gamma_{00}}\left(1+e^{-\gamma_{00}}\right)^{-2}\left[1+\tau_{0}^{2}(K-1) e^{-\gamma_{00}}\left(1+e^{-\gamma_{00}}\right)^{-2}\right]
$$

Com a inclusão do efeito aleatório $\varepsilon_{0 j}$ no Modelo 2, leva-se em conta a possível dependência entre as respostas de alunos da mesma escola, explicando-se ao menos parte da suposta superdispersão presente no Modelo 1. A covariância e a correlação marginais para os escores de alunos de uma mesma escola são dadas, respectivamente, por

$$
\operatorname{Cov}\left[Y_{i j}, Y_{i^{\prime} j}\right] \cong\left[K \tau_{0} e^{-\gamma_{00}}\left(1+e^{-\gamma_{00}}\right)^{-2}\right]^{2}
$$

e

$$
\operatorname{Corr}\left[Y_{i j}, Y_{i^{\prime} j}\right] \cong K \tau_{0}^{2}\left\{\left[e^{-\gamma_{00}}\left(1+e^{-\gamma_{00}}\right)^{-2}\right]^{-1}+\tau_{0}^{2}(K-1)\right\}^{-1}
$$

A correlação em (1.17), conhecida como correlação intra-classe, será sempre positiva. Esta pode ser interpretada como o grau de dependência ou de similaridade entre as respostas de alunos da mesma escola.

As expressões das esperanças, variâncias, covariâncias e correlações marginais de $p_{i j}$ e de $Y_{i j}$, apresentadas de (1.12) a (1.17), podem ser obtidas a partir do Apêndice A. Neste apêndice, um vetor de funções $\boldsymbol{G}_{j}\left(\boldsymbol{b}_{j}\right)$ é aproximado, por meio de uma expansão de Taylor de primeira ordem 
em torno da média de $\boldsymbol{b}_{j}$, que é o vetor de efeitos aleatórios da escola $j$ e de seus $n_{j}$ alunos. Vale ressaltar que as expressões do Apêndice A são mais amplas do que as apresentadas ao longo deste capítulo, pois admitem efeitos aleatórios não gaussianos, bem como a inclusão de covariáveis cujos efeitos sejam fixos.

Os parâmetros do Modelo 2 podem ser estimados por técnicas apropriadas para o ajuste de um MLGH binomial-normal (veja Raudenbush e Bryk (2002), por exemplo), ou equivalentemente por técnicas de um MLGM binomial (veja Molenberghs e Verbeke (2005), por exemplo). Note que o Modelo 1 é retomado se $\tau_{0}^{2}=0$.

Mesmo com a inclusão do efeito aleatório $\varepsilon_{0 j}$, ainda pode haver uma variabilidade extra causada pelos problemas 1c e 1d, supondo que não haja covariáveis que dêem conta desta variabilidade adicional. Introduzindo um parâmetro de superdispersão $\phi$, tem-se $\operatorname{Var}\left[Y_{i j} \mid p_{i j}\right]=\phi K p_{i j}\left(1-p_{i j}\right)$, de modo que a esperança e a covariância marginais permanecem iguais a (1.14) e (1.16), respectivamente, mas a variância e a correlação marginais de $Y_{i j}$ ficam então expressas por

$$
\operatorname{Var}\left[Y_{i j}\right] \cong K e^{-\gamma_{00}}\left(1+e^{-\gamma_{00}}\right)^{-2}\left[\phi+\tau_{0}^{2}(K-\phi) e^{-\gamma_{00}}\left(1+e^{-\gamma_{00}}\right)^{-2}\right]
$$

e

$$
\operatorname{Corr}\left[Y_{i j}, Y_{i^{\prime} j}\right] \cong K \tau_{0}^{2}\left\{\phi\left[e^{-\gamma_{00}}\left(1+e^{-\gamma_{00}}\right)^{-2}\right]^{-1}+\tau_{0}^{2}(K-\phi)\right\}^{-1}
$$

Em vez de tentar corrigir a superdispersão ainda existente com o parâmetro de superdispersão $\phi$, será atribuído um efeito aleatório para cada aluno. Então, propõe-se o Modelo 3 :

\section{Modelo 3.}

$$
\begin{aligned}
& \text { Nível 1: } \quad \log \left(\frac{p_{i j}}{1-p_{i j}}\right)=\beta_{0 j}+a_{i j}, \\
& a_{i j} \sim N\left(0, \sigma^{2}\right) \text { i.i.d. }
\end{aligned}
$$

e

$$
\begin{aligned}
& \text { Nível } 2: \quad \beta_{0 j}=\gamma_{00}+\varepsilon_{0 j}, \\
& \gamma_{00} \text { fixo, } \varepsilon_{0 j} \sim N\left(0, \tau_{0}^{2}\right) \text { i.i.d., } \varepsilon_{0 j} \text { e } a_{i j} \text { independentes. }
\end{aligned}
$$

Sob este modelo 


$$
\begin{aligned}
\mathrm{E}\left[Y_{i j} \mid p_{i j}\right] & =K p_{i j} \\
\operatorname{Var}\left[Y_{i j} \mid p_{i j}\right] & =K p_{i j}\left(1-p_{i j}\right), \\
\mathrm{E}\left[p_{i j}\right] & \cong\left(1+e^{-\gamma_{00}}\right)^{-1}
\end{aligned}
$$

$\mathrm{e}$

$$
\operatorname{Var}\left[p_{i j}\right] \cong\left(\tau_{0}^{2}+\sigma^{2}\right)\left[e^{-\gamma_{00}}\left(1+e^{-\gamma_{00}}\right)^{-2}\right]^{2}
$$

Assim, a esperança e a variância de $Y_{i j}$ são

$$
\mathrm{E}\left[Y_{i j}\right] \cong K\left(1+e^{-\gamma_{00}}\right)^{-1}
$$

$\mathrm{e}$

$$
\operatorname{Var}\left[Y_{i j}\right] \cong K e^{-\gamma_{00}}\left(1+e^{-\gamma_{00}}\right)^{-2}\left[1+\left(\tau_{0}^{2}+\sigma^{2}\right)(K-1) e^{-\gamma_{00}}\left(1+e^{-\gamma_{00}}\right)^{-2}\right]
$$

Nesse caso a covariância e a correlação marginais para os escores de alunos de uma mesma escola são

$$
\operatorname{Cov}\left[Y_{i j}, Y_{i^{\prime} j}\right] \cong\left[K \tau_{0} e^{-\gamma_{00}}\left(1+e^{-\gamma_{00}}\right)^{-2}\right]^{2}
$$

$\mathrm{e}$

$$
\operatorname{Corr}\left[Y_{i j}, Y_{i^{\prime} j}\right] \cong K \tau_{0}^{2}\left\{\left[e^{-\gamma_{00}}\left(1+e^{-\gamma_{00}}\right)^{-2}\right]^{-1}+\left(\tau_{0}^{2}+\sigma^{2}\right)(K-1)\right\}^{-1}
$$

As deduções de (1.24) a (1.29) foram obtidas como casos particulares das expressões do Apêndice A.

Observe que a expressão da covariância marginal (1.28) é igual a de (1.16), mas o cálculo da variância marginal por (1.27) leva a valores superiores aos obtidos por (1.15). Conseqüentemente, a correlação marginal calculada por (1.29) é inferior à obtida por (1.17), o que significa que a introdução dos efeitos aleatórios $a_{i j}$ reduz a correlação intra-classe.

Analogamente ao Modelo 2, em que os efeitos aleatórios $\varepsilon_{0 j}$ induzem uma correlação entre as respostas dos alunos da mesma escola, os efeitos aleatórios $a_{i j}$ no Modelo 3 induzem uma dependência entre as respostas aos $K$ itens dadas pelo mesmo aluno, de modo a contemplar o problema 1c, que será avaliado neste trabalho. 
Caso ainda haja variação extra-binomial após a inclusão de efeitos aleatórios em ambos os níveis do modelo, esta possivelmente é causada pelo problema 1d. Para acomodar esta variabilidade remanescente, pode-se novamente utilizar como abordagem um fator $\phi$ multiplicando a variância condicional da resposta $Y_{i j}$ no Modelo 3, que não altera as respectivas expressões da esperança e da covariância em (1.26) e em (1.28), mas modifica as expressões da variância e da correlação marginais para escores de alunos da mesma escola, dadas por

$$
\operatorname{Var}\left[Y_{i j}\right] \cong K e^{-\gamma_{00}}\left(1+e^{-\gamma_{00}}\right)^{-2}\left[\phi+\left(\tau_{0}^{2}+\sigma^{2}\right)(K-\phi) e^{-\gamma_{00}}\left(1+e^{-\gamma_{00}}\right)^{-2}\right]
$$

$\mathrm{e}$

$$
\operatorname{Corr}\left[Y_{i j}, Y_{i^{\prime} j}\right] \cong K \tau_{0}^{2}\left\{\phi\left[e^{-\gamma_{00}}\left(1+e^{-\gamma_{00}}\right)^{-2}\right]^{-1}+\left(\tau_{0}^{2}+\sigma^{2}\right)(K-\phi)\right\}^{-1}
$$

A estimação do parâmetro de superdispersão $\phi$ neste último caso não será alvo de investigação deste trabalho e ficará como estudo futuro.

Embora haja uma vasta discussão sobre as vantagens e desvantagens de diferentes propostas de estimação e implementações computacionais, poucos trabalhos da literatura tratam especificamente da superdispersão em MLGHs. A superdispersão é mais comumente tratada sob o enfoque de MLGMs, não necessariamente para dados de natureza hierárquica. Muitos trabalhos utilizam a introdução de efeitos aleatórios como forma de acomodar a superdispersão (veja, por exemplo, Molenberghs e Verbeke (2005)). Uma outra alternativa é o uso de modelos beta-binomiais (veja Hinde e Demétrio (1998), por exemplo). As causas mais freqüentes de superdispersão em MLGHs citadas na literatura são a heterogeneidade de variâncias, a correlação entre respostas, níveis insuficientes de modelagem e omissão de preditores importantes. No caso de MLGHs, a própria natureza dos dados justifica o uso de efeitos aleatórios e mesmo após se levar em conta a dependência entre unidades pertencentes ao mesmo grupo, ainda pode ocorrer superdispersão, pelos motivos já citados.

Efron (1986) introduz o uso de famílias exponenciais duplas para modelar simultaneamente a média e a variação extra-binomial por meio de variáveis explicativas. Lee e Nelder (2006) e Lee, Nelder e Pawitan (2006), propõem uma extensão destes modelos com os chamados MLGHs duplos, que permitem até mesmo o uso de efeitos aleatórios no parâmetro de dispersão, porém são utilizados apenas efeitos aleatórios associados a grupos de unidades experimentais (nível 2, por exemplo).

Quanto aos processos de estimação para os MLGHs, a literatura discute diferentes métodos, 
com maior enfoque nos MLGMs em que se supõem efeitos aleatórios normalmente distribuídos. Goldstein (1991), Wolfinger e O'Connell (1993) e Breslow e Clayton (1993), utilizam o método de quase-verossimilhança penalizada (QVP), muito criticado pelos estimadores enviesados e inconsistentes que produz (veja Molenberghs e Verbeke (2005), por exemplo). Diante das desvantagens deste método, Breslow e Lin (1995), Lin e Breslow (1996) e Raudenbush, Yang e Yosef (2000) sugerem o uso de aproximação de Laplace para obter melhores propriedades. Lee e Nelder (1996) estendem os MLGHs com o uso de efeitos aleatórios com distribuição na família exponencial, por eles denominada de "distribuição conjugada" (em relação à distribuição marginal da resposta considerada), referindo-se à situação em que se obtém uma função de verossimilhança com expressão analítica conhecida, dispensando o uso de métodos de aproximação. Anderson e Aitkin (1985), Hedeker e Gibbons (1994) e Davidian e Giltinan (1995) aplicam o método de quadratura de GaussHermite. Pinheiro e Bates (1995) destacam a eficiência computacional do método de quadratura gaussiana adaptativa para aproximar a função de verossimilhança, em relação àqueles em que se assumem efeitos aleatórios normalmente distribuídos. No entanto, o seu uso torna-se mais complicado à medida em que se aumenta o número de efeitos aleatórios. A literatura apresenta como alternativa alguns métodos computacionalmente intensos, como o método MCMC (Markov Chain Monte Carlo) com amostradores de Gibbs ou Metropolis Hastings (veja por exemplo Zeger e Karim (1991) e Goldstein (2003)).

A maioria dos trabalhos em MLGHs que falam em variação extra-binomial apenas sugerem acomodá-la com a inclusão de um parâmetro de superdispersão (veja Goldstein (2003) e Jacob (2000), por exemplo). A literatura de MLGs não apresenta nenhum trabalho em que o efeito aleatório associado ao nível 1 tenha sido incorporado aditivamente ao preditor linear da média e ao mesmo tempo que um efeito aleatório oriundo de outro nível da hierarquia.

O objetivo deste trabalho é avaliar se o Modelo 3 proposto é superior aos Modelos 1 e 2, produzindo estimadores menos enviesados para os parâmetros comuns, erros padrões que contemplam mais apropriadamente a variabilidade dos dados e, níveis de significância empíricos mais adequados para testes de hipótese do parâmetro $\gamma_{00}$. Para tanto foi desenvolvido um programa computacional que estima os parâmetros de um MLGH sob o Modelo 3 e foi conduzido um estudo de simulação no software $\mathrm{R}$ de modo a comparar os resultados sob os Modelos 1, 2 e 3, supondo que os efeitos aleatórios $\varepsilon_{0 j}$ e $a_{i j}$ sejam normalmente distribuídos e independentes.

As simulações foram feitas de modo que todas as amostras geradas sejam avaliadas sob o 
Modelo 1 (equações (1.5) e (1.6)) por máxima verossimilhança (MV) e por quase-verossimilhança (QV) e, em seguida, sob os Modelos 2 (equações (1.10) e (1.11)) e 3 (equações (1.20) e (1.21)), por MV associada ao uso do método de quadratura de Gauss-Hermite adaptativa (QGHA), que é o método de quadratura de Gauss-Hermite com uma modificação na escolha dos pontos de quadratura (veja Liu e Pierce (1994), por exemplo).

O Capítulo 2 descreve a estratégia de simulação, a teoria do método de QGHA e as ferramentas de maximização. O Capítulo 3 apresenta os resultados do estudo de simulação. O Capítulo 4 mostra uma análise de dados reais na área de educação e no Capítulo 5 são feitas as conclusões deste trabalho. 


\section{Capítulo 2}

\section{Descrição do estudo de simulação}

\subsection{Geração de dados}

De acordo com o Modelo 3 tem-se

$$
\text { Nível } 1: \quad \log \left(\frac{p_{i j}}{1-p_{i j}}\right)=\beta_{0 j}+a_{i j}
$$

e

$$
\text { Nível 2: } \quad \beta_{0 j}=\gamma_{00}+\varepsilon_{0 j} \text {, }
$$

em que $a_{i j}$ e $\varepsilon_{0 j}$ são independentes e normalmente distribuídos, com esperanças nulas e variâncias iguais a $\sigma^{2}$ e $\tau_{0}^{2}$, respectivamente, $i=1,2, \ldots, n_{j}$ e $j=1,2, \ldots, J$.

Considere as variáveis $z_{a_{i j}}$ e $z_{\varepsilon_{0 j}}$, de modo que $a_{i j}=\sigma z_{a_{i j}}$ e $\varepsilon_{0 j}=\tau_{0} z_{\varepsilon_{0 j}}$.

Combinando os dois níveis do modelo e substituindo $a_{i j}$ e $\varepsilon_{0 j}$ por suas versões padronizadas, os desvios padrões (DPs) dos efeitos aleatórios tornam-se parâmetros explícitos no preditor linear:

$$
\log \left(\frac{p_{i j}}{1-p_{i j}}\right)=\gamma_{00}+\tau_{0} z_{\varepsilon_{0 j}}+\sigma z_{a_{i j}} .
$$

Suponha que em uma prova composta de $K$ itens, o total de acertos $Y_{i j}$ que o $i$-ésimo aluno 
da $j$-ésima escola obtém tenha uma distribuição binomial com parâmetros $K$ e $p_{i j}$. Para gerar $Y_{i j}$, são executados os seguintes passos:

1. fixam-se valores para os parâmetros populacionais, ou seja, $\boldsymbol{\theta}=\left(\gamma_{00}, \tau_{0}, \sigma\right)^{T}$;

2. gera-se uma a.a. de tamanho $J$ da variável $z_{\varepsilon_{0 j}} \sim N(0,1)$;

3. para cada $z_{\varepsilon_{0 j}}$ obtido, gera-se uma a.a. de tamanho $n_{j}$ da variável $z_{a_{i j}} \sim N(0,1)$, supondo que $z_{\varepsilon_{0 j}}$ e $z_{a_{i j}}$ são independentes;

4. substituem-se os valores obtidos nos passos anteriores em (2.2) e calcula-se $p_{i j}$;

5. para cada $p_{i j}$, gera-se um valor da variável $Y_{i j} \sim \operatorname{Bin}\left(K, p_{i j}\right)$.

Tem-se agora para cada uma das $J$ escolas, uma amostra do total de acertos para $n_{j}$ alunos.

\subsection{Delineamento do estudo de simulação}

Os parâmetros foram estimados em cada amostra segundo cinco diferentes ajustes. Os dois primeiros foram ajustes do Modelo 1, um sob MV e outro sob QV. O terceiro e o quarto foram ajustes do Modelo 2 e o último foi um ajuste do Modelo 3, todos estes sob MV e com o uso de QGHA. Mais detalhes sobre a QGHA e sobre os ajustes estão nas Seções 2.3 e 2.4 respectivamente.

As simulações foram realizadas considerando 18 diferentes populações, caracterizadas por diferentes combinações de valores dos parâmetros $\gamma_{00}, \tau_{0}$ e $\sigma$. O efeito fixo $\gamma_{00}$ foi avaliado apenas nos valores 0 e 1 , que correspondem a $p_{i j}=0.5$ e $p_{i j} \cong 0.73$ respectivamente. Em virtude da enorme demanda computacional, não foi possível avaliar $p_{i j}$ para outros valores de $\gamma_{00}$. Pelo mesmo motivo, os componentes de variância $\tau_{0}$ e $\sigma$ foram avaliados apenas nos valores $0,0.5$ e 1 . Estes valores foram escolhidos de modo que fossem contemplados casos em que $\left(\tau_{0}=0, \sigma=0\right),\left(\tau_{0}=0, \sigma>0\right)$, $\left(\tau_{0}>0, \sigma=0\right)$ e $\left(\tau_{0}>0, \sigma>0\right)$ e que resultassem em distribuições variadas mas razoáveis para $p_{i j}$ numa aplicação real. Note que

$$
\log \left(\frac{p_{i j}}{1-p_{i j}}\right) \sim N\left(\gamma_{00}, \tau_{0}^{2}+\sigma^{2}\right)
$$


resultando na distribuição logística-normal para a escala das probabilidades, cuja função densidade de probabilidade (f.d.p.) é

$$
f\left(p_{i j} \mid \gamma_{00}, \tau_{0}, \sigma\right)=\frac{\left[p_{i j}\left(1-p_{i j}\right)\right]^{-1}}{\sqrt{2 \pi\left(\tau_{0}^{2}+\sigma^{2}\right)}} \exp \left\{-\frac{1}{2\left(\tau_{0}^{2}+\sigma^{2}\right)}\left[\log \left(\frac{p_{i j}}{1-p_{i j}}\right)-\gamma_{00}\right]^{2}\right\}
$$

Foram calculadas a esperança e a variância de $p_{i j}$ aproximadas de acordo com as expressões (1.24) e (1.25), respectivamente. Estas medidas foram aproximadas também, considerando probabilidades atribuídas no intervalo de 0.0001 a 0.9999 , a cada 0.0001 unidade, ponderadas pela f.d.p. logísticanormal. Esta aproximações, que serão identificadas por Aprox- $B$, foram contrastadas com as obtidas pelas respectivas expressões (1.24) e (1.25), identificadas por Aprox-A. A comparação dos resultados obtidos encontra-se na Tabela 2.1, em que as aproximações em Aprox- $A$ distanciam-se das obtidas em Aprox-B, com o aumento de $\tau_{0}^{2}+\sigma^{2}$, conforme comentado no Apêndice A.1, principalmente nos casos em que $\tau_{0}^{2}+\sigma^{2}>0.5$. As densidades de $p_{i j}$ em que $\tau_{0}^{2}+\sigma^{2}>0$ são ilustradas na Figura 2.1, uma vez que $\tau_{0}=\sigma=0$ resulta numa distribuição degenerada.

Tabela 2.1: Medidas resumo das distribuições de $p_{i j}$

\begin{tabular}{|c|c|c|c|c|c|c|}
\hline \multirow{2}{*}{$\left(\gamma_{00}, \tau_{0}, \sigma\right)$} & \multirow{2}{*}{$\tau_{0}^{2}+\sigma^{2}$} & \multicolumn{2}{|c|}{$\mathrm{E}\left(p_{i j}\right)$} & \multicolumn{2}{|c|}{$D P\left(p_{i j}\right)$} & \multirow{2}{*}{$\begin{array}{l}\text { Intervalo de } 90 \% \text { de } \\
\text { densidade máxima }\end{array}$} \\
\hline & & Aprox- $B$ & Aprox-A & Aprox-B & Aprox-A & \\
\hline$(0,0,0)$ & 0 & 0.500 & 0.500 & 0.000 & 0.000 & \\
\hline$(0,0,0.5)$ ou $(0,0.5,0)$ & 0.25 & 0.500 & 0.500 & 0.118 & 0.125 & $0.305 ; 0.695$ \\
\hline$(0,0.5,0.5)$ & 0.5 & 0.500 & 0.500 & 0.159 & 0.177 & $0.238 ; 0.768$ \\
\hline$(0,0,1)$ ou $(0,1,0)$ & 1 & 0.500 & 0.500 & 0.208 & 0.250 & $0.162 ; 0.838$ \\
\hline$(0,0.5,1)$ ou $(0,1,0.5)$ & 1.25 & 0.500 & 0.500 & 0.225 & 0.280 & $0.137 ; 0.863$ \\
\hline$(0,1,1)$ & 2 & 0.500 & 0.500 & 0.262 & 0.354 & $0.089 ; 0.911]$ \\
\hline$(1,0,0)$ & 0 & 0.731 & 0.731 & 0.000 & 0.000 & \\
\hline$(1,0,0.5)$ ou $(1,0.5,0)$ & 0.25 & 0.721 & 0.731 & 0.097 & 0.098 & $0.568 ; 0.878$ \\
\hline$(1,0.5,0.5)$ & 0.5 & 0.712 & 0.731 & 0.135 & 0.139 & $0.505 ; 0.924$ \\
\hline$(1,0,1)$ ou $(1,1,0)$ & 1 & 0.697 & 0.731 & 0.183 & 0.197 & $0.419 ; 0.969$ \\
\hline$(1,0.5,1)$ ou $(1,1,0.5)$ & 1.25 & 0.690 & 0.731 & 0.200 & 0.220 & $0.386 ; 0.980$ \\
\hline$(1,1,1)$ & 2 & 0.675 & 0.731 & 0.239 & 0.278 & $0.305 ; 0.995$ \\
\hline
\end{tabular}

Aprox- $A$ : refere-se aos resultados oriundos da esperança (1.24) e variância (1.25).

Aprox-B: identifica os resultados obtidos por meio de uma distribuição logística-normal.

Para cada uma das 18 populações considerou-se o número de grupos $J=20,50$ combinado com o número de unidades no $j$-ésimo grupo $n_{j}=20,50, j=1, \ldots, J$, totalizando 72 diferentes configurações de valores de parâmetros com tamanhos de amostra. Os valores atribuídos para $J$ e 


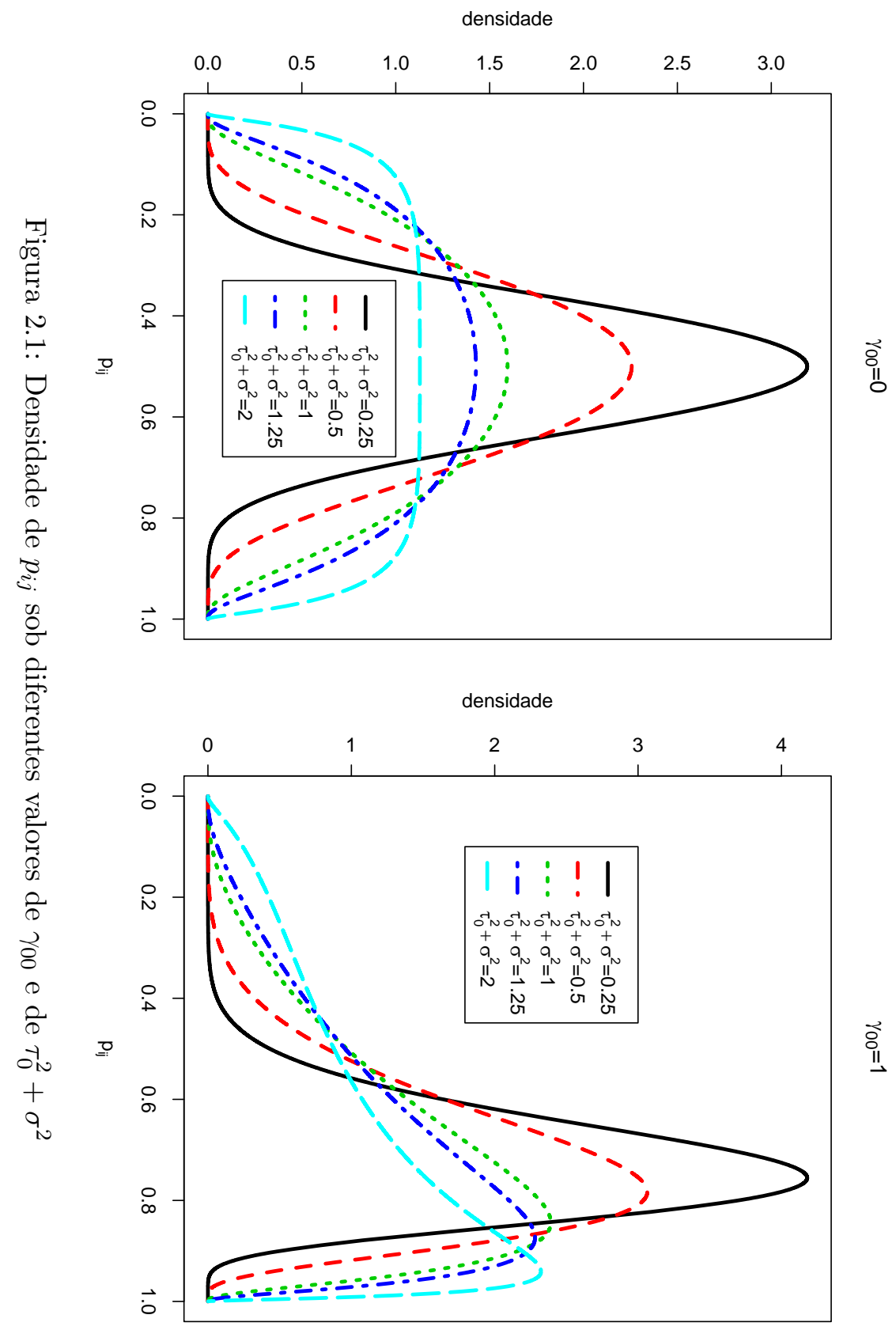


$n_{j}$ foram escolhidos levando-se em conta: 1) o que seria razoável em uma aplicação real, como por exemplo alunos agrupados em suas escolas, 2) o crescimento da demanda computacional à medida que $J$ e $n_{j}$ aumentam (a quadratura Gaussiana adaptativa, por exemplo, será empregada $J$ vezes

e $J+\sum_{j=1}^{J} n_{j}$ vezes nos modelos 2 e 3 , respectivamente) e 3) o interesse em avaliar mudanças nos resultados com o aumento no número de escolas e/ou no número de alunos.

A partir de cada uma das 72 configurações, foram geradas pelo menos $1000 \operatorname{amostras}^{1}$ (ou réplicas). A principal dificuldade em diversificar os valores dos parâmetros, tamanhos de $J$ e $n_{j}$ e em aumentar o número de réplicas, consistiu no grande número de horas gastas no trabalho computacional. Para viabilizar o estudo, as simulações foram divididas em dezenas de processadores, conforme eles eram disponibilizados para uso. O trabalho computacional ficou distribuído da seguinte maneira: 42\% num Cluster IBM com 112 nós com 4 CPUs (PowerPC 970) de 2.5GHz cada, um supercomputador pertencente ao projeto FAPESP No. 04/08928-3; 47\% num Cluster Intel Dual Xeon com 32 nós de $2.4 \mathrm{GHz}$ cada, pertencente ao LCCA-USP (Laboratório de Computação Científica Avançada-USP); $11 \%$ em computadores do IME-USP e em notebooks de uso pessoal, com configurações próximas às de um Intel Core Duo 1.66GHz.

Para avaliar as réplicas geradas de cada uma das 18 populações, o tempo de processamento variou de 8 a 29 dias para $J=n_{j}=20,16$ a 92 dias para $J=20$ e $n_{j}=50,16$ a 90 dias para $J=50$ e $n_{j}=20$ e 40 a 122 dias para $J=n_{j}=50$, ou seja, à medida que o tamanho da amostra aumentou, o processamento ficou mais demorado, como era esperado. As simulações para as 72 configurações estabelecidas totalizaram 3392 dias ( $\cong 9.3$ anos) de trabalho computacional, somando-se todas as horas gastas em todos os processadores.

\subsection{Método de quadratura de Gauss-Hermite adaptativa}

Para a avaliação do Modelo 3 foi utilizado o método de quadratura de Gauss-Hermite adaptativa (QGHA). Por meio dele foi possível aproximar as integrais requeridas nos dois níveis do modelo (aluno e escola) para obtenção da verossimilhança marginal das respostas. No caso do Modelo 2, o emprego da QGHA se dá apenas no segundo nível (escola) e constitui um caso particular do que

\footnotetext{
${ }^{1}$ Valores maiores que 1000 ocorreram devido à divisão da simulação de uma mesma configuração em dois ou mais processadores. Os valores 1015, 1074, 1344 e 1841 correspondem ao $1^{\circ}$ quartil, mediana, $3^{\circ}$ quartil e máximo para o número de réplicas, respectivamente.
} 
será descrito a seguir.

A f.d.p. de $Y_{i j}$ condicional à $p_{i j}$ é dada por:

$$
f\left(y_{i j} \mid p_{i j}\right) \propto \exp \left[y_{i j} \log \left(\frac{p_{i j}}{1-p_{i j}}\right)+K \log \left(1-p_{i j}\right)\right]
$$

Utilizando (2.2) em (2.3) tem-se:

$f\left(y_{i j} \mid z_{\varepsilon_{0 j}}, z_{a_{i j}}, \boldsymbol{\theta}\right) \propto \exp \left[y_{i j}\left(\gamma_{00}+\tau_{0} z_{\varepsilon_{0 j}}+\sigma z_{a_{i j}}\right)-K \log \left(1+e^{\gamma_{00}+\tau_{0} z_{\varepsilon_{0 j}}+\sigma z_{a_{i j}}}\right)\right]$.

O logaritmo da função de verossimilhança marginal de $\boldsymbol{Y}$ é dada por

$$
\log L(\boldsymbol{\theta} ; \boldsymbol{Y})=\sum_{j=1}^{J} \log \left\{\int_{z_{\varepsilon_{0 j}}}\left[\prod_{i=1}^{n_{j}} \int_{z_{a_{i j}}} f\left(y_{i j} \mid z_{\varepsilon_{0 j}}, z_{a_{i j}}, \boldsymbol{\theta}\right) f\left(z_{a_{i j}}\right) d z_{a_{i j}}\right] f\left(z_{\varepsilon_{0 j}}\right) d z_{\varepsilon_{0 j}}\right\} .
$$

A integral da função $f\left(y_{i j} \mid z_{\varepsilon_{0 j}}, z_{a_{i j}}, \boldsymbol{\theta}\right) f\left(z_{a_{i j}}\right)$ na variável $z_{a_{i j}}$ pode ser aproximada pelo método de quadratura de Gauss-Hermite (veja Liu e Pierce (1994), por exemplo), de modo que:

$$
\int_{z_{a_{i j}}} f\left(y_{i j} \mid z_{\varepsilon_{0 j}}, z_{a_{i j}}, \boldsymbol{\theta}\right) f\left(z_{a_{i j}}\right) d z_{a_{i j}} \cong \sum_{q=1}^{Q_{a}} w^{*}\left(z_{a_{i j}}^{(q)}\right) f\left(y_{i j} \mid z_{\varepsilon_{0 j}}, z_{a_{i j}}^{(q)}, \boldsymbol{\theta}\right) f\left(z_{a_{i j}}^{(q)}\right)
$$

em que $Q_{a}$ corresponde ao número de pontos de quadratura utilizados nesta aproximação, ou seja, ao grau do polinômio de Hermite. Cada raiz $z_{a_{i j}}^{(q)}$ do polinômio de Hermite, $q=1, \ldots, Q_{a}$, será avaliada na função $w^{*}(z)=w(z) \exp \left(z^{2}\right)$, sendo $w(z)$ uma função ortogonal ao polinômio de Hermite. 
Analogamente aproxima-se a integral em $z_{\varepsilon_{0 j}}$

$$
\begin{aligned}
& \int_{z_{\varepsilon_{0 j}}}\left[\prod_{i=1}^{n_{j}} \int_{z_{a_{i j}}} f\left(y_{i j} \mid z_{\varepsilon_{0 j}}, z_{a_{i j}}, \boldsymbol{\theta}\right) f\left(z_{a_{i j}}\right) d z_{a_{i j}}\right] f\left(z_{\varepsilon_{0 j}}\right) d z_{\varepsilon_{0 j}} \\
& \cong \int_{z_{\varepsilon_{0 j}}}\left[\prod_{i=1}^{n_{j}}\left(\sum_{q=1}^{Q_{a}} w^{*}\left(z_{a_{i j}}^{(q)}\right) f\left(y_{i j} \mid z_{\varepsilon_{0 j}}, z_{a_{i j}}^{(q)}, \boldsymbol{\theta}\right) f\left(z_{a_{i j}}^{(q)}\right)\right)\right] f\left(z_{\varepsilon_{0 j}}\right) d z_{\varepsilon_{0 j}} \\
& \cong \sum_{q^{\prime}=1}^{Q_{\varepsilon}} w^{*}\left(z_{\varepsilon_{0 j}}^{\left(q^{\prime}\right)}\right)\left[\prod_{i=1}^{n_{j}}\left(\sum_{q=1}^{Q_{a}} w^{*}\left(z_{a_{i j}}^{(q)}\right) f\left(y_{i j} \mid z_{\varepsilon_{0 j}}^{\left(q^{\prime}\right)}, z_{a_{i j}}^{(q)}, \boldsymbol{\theta}\right) f\left(z_{\left.a_{i j}\right)}^{(q)}\right)\right] f\left(z_{\varepsilon_{0 j}}^{\left(q^{\prime}\right)}\right)\right.
\end{aligned}
$$

em que $z_{\varepsilon_{0 j}}\left(q^{\prime}\right), q^{\prime}=1, \ldots, Q_{\varepsilon}$ constituem as raízes do polinômio de Hermite de grau $Q_{\varepsilon}$, avaliadas em $w^{*}(z)=w(z) \exp \left(z^{2}\right)$, de modo que

$$
\log L(\boldsymbol{\theta} ; \boldsymbol{Y}) \cong \sum_{j=1}^{J} \log \left\{\sum_{q^{\prime}=1}^{Q_{\varepsilon}} w^{*}\left(z_{\varepsilon_{0 j}}^{\left(q^{\prime}\right)}\right)\left[\prod_{i=1}^{n_{j}}\left(\sum_{q=1}^{Q_{a}} w^{*}\left(z_{a_{i j}}^{(q)}\right) f\left(y_{i j} \mid z_{\varepsilon_{0 j}}^{\left(q^{\prime}\right)}, z_{a_{i j}}^{(q)}, \boldsymbol{\theta}\right) f\left(z_{a_{i j}}^{(q)}\right)\right)\right] f\left(z_{\varepsilon_{0 j}}^{\left(q^{\prime}\right)}\right)\right\}
$$

Como os pontos de quadratura são escolhidos independentemente da função que se deseja integrar, estes podem estar em regiões que pouco contribuem no resultado da integração desta função. Para melhorar a aproximação, é conveniente promover uma mudança de localização e escala nos pontos de quadratura de forma a deslocá-los para regiões que promovam maior contribuição. Esta modificação resulta no método de QGHA. Segundo Liu e Pierce (1994), os pontos de quadratura da QGHA são reposicionados em torno de $\hat{\mu}$, a moda estimada da função $h(z)$ a ser integrada, utilizando-se como parâmetro de escala

$$
\hat{\nu}=\left[-\left.\frac{\partial^{2} \log h(z)}{\partial z^{2}}\right|_{z=\hat{\mu}}\right]^{-1 / 2}
$$

Desta forma a função $h(z)$ pode ser aproximada por

$$
\int h(z) d z \cong \sqrt{2} \hat{\nu} \sum_{q=1}^{Q} w^{*}\left(z^{(q)}\right) h\left(\hat{\mu}+\sqrt{2} \hat{\nu} z^{(q)}\right)
$$

em que $w^{*}(z)=w(z) \exp \left(z^{2}\right)$. 


\subsection{Implementação no R}

Para tornar a implementação computacional deste trabalho acessível a todos os interessados em utilizá-la, optou-se pelo uso do software livre $\mathrm{R}$, disponível em The $R$ Project for Statistical Computing, no sítio http://www.r-project.org.

Dois ajustes do Modelo 1 foram feitos pela rotina glm do software R, utilizando a opção family=quasibinomial, que disponibiliza tanto os erros padrões (EPs) do ajuste de um MLG quanto os EPs corrigidos pelo parâmetro de superdispersão estimado. Os ajustes do Modelo 1 sob MV e sob QV serão identificados pelas respectivas siglas MLG e QV.

O ajuste do Modelo 2 foi feito por meio da rotina glmmML do R e será identificado pela sigla QGR. Neste caso as estimativas dos EPs são obtidas por meio da matriz de informação observada analítica. Utilizou-se a opção method=' 'ghq' ', em que a rotina faz uso da QGHA na estimação por MV. Outro ajuste do Modelo 2 sob MV e com o uso da QGHA, que será identificado por QG2, foi obtido por meio de um programa feito no R. Este último ajuste utiliza uma forma reduzida do programa que a autora desenvolveu no R, para o ajuste do Modelo 3, que será identificado pela sigla QG3, tendo como objetivo intermediar os resultados de QGR e QG3, avaliando assim a adequação do algoritmo implementado em QG3. Todas as aplicações de QGHA envolveram 10 pontos de quadratura $\left(Q_{a}=Q_{\varepsilon}=10\right)$. Tanto em QG2 como em QG3 as estimativas dos EPs foram obtidas por meio da matriz de informação observada numérica. Tomou-se esta decisão com o objetivo de evitar o aumento do tempo computacional, visto que um estudo piloto não apontou diferenças expressivas entre os resultados obtidos com a aproximação numérica e seus correspondentes com a expressão analítica.

A QGHA em QG3 foi implementada em duas etapas, aproximando as integrais das funções de $z_{a_{i j}}$ e, posteriormente, das funções de $z_{\varepsilon_{0 j}}$. Para a primeira etapa, a QGHA teve os pontos de quadratura selecionados em torno da estimativa da moda $\mu$, calculada para a função integrada utilizando a rotina de otimização unidimensional optimize, mais rápida do que a rotina optim, já que havia $\sum_{j=1}^{J} n_{j}$ integrais a serem aproximadas. A estimativa do parâmetro de escala $\nu$ da equação (2.9) foi obtida, posteriormente, com o emprego de $\mu$ na expressão analítica da hessiana obtida pela rotina deriv3. Já para a segunda etapa, que requer a aproximação de $J$ integrais, as estimativas de $\mu$ e de $\nu$ para a função integrada foram obtidas pela rotina optim, que disponibiliza 
a matriz hessiana numérica. Em alguns poucos casos foi preciso utilizar outra rotina de otimização denominada nlm, que utiliza um algoritmo tipo-Newton. Veja mais detalhes das rotinas do software R no sítio http://www.r-project.org.

A rotina optim permite especificar diferentes métodos de otimização, entre os quais tem-se: 1) Nelder-Mead, default da rotina, método simplex proposto em Nelder e Mead (1965); 2) BFGS, um método quasi-Newton; 3) L-BFGS-B, uma adaptação de BFGS em que cada parâmetro pode ficar restrito a um intervalo especificado; 4) CG, um método de gradientes conjugados que, segundo o manual do R, é mais frágil do que o método BFGS mas pode ter mais êxito em problemas de otimização maiores. Detalhes sobre estes últimos métodos podem ser encontrados, por exemplo, em Nocedal e Wright (1999).

Uma vez feitas as aproximações das integrais pela QGHA, tanto em QG2 quanto em QG3, cada amostra teve sua função de verossimilhança maximizada pela rotina optim com a opção Nelder-Mead. Em caso de problemas de convergência ou de obtenção de estimativas negativas para a variância dos estimadores, optou-se pelos métodos L-BFGS-B ou BFGS, nesta ordem.

Pela Tabela 2.2, vê-se que em todas as situações, mais de $95 \%$ das amostras tiveram sua função de verossimilhança avaliada pelo mesmo método de otimização (Nelder-Mead). Na obtenção da moda da função a ser integrada em $z_{\varepsilon_{0 j}}$ (veja equação $(2.7)$ ), novamente em mais de $95 \%$ das amostras os resultados foram originados pelo mesmo método de otimização (CG). Os outros métodos foram empregados nos casos em que ocorreram problemas de convergência, de forma que os algoritmos fossem finalizados e não se desprezasse nenhuma das amostras. Apenas 0.09\% das amostras tiveram estimativas em QG2 ou QG3 desprezadas por apresentarem estimativas negativas de componentes de variância.

Tabela 2.2: Percentual dos métodos de otimização usados em QG2 e QG3, na maximização da função de verossimilhança e da função $h\left(z_{\varepsilon_{0 j}}\right)$ na QGHA.

\begin{tabular}{|c|c|c|c|c|c|}
\hline Método de & \multicolumn{2}{|c|}{ F. de verossimilhança } & Método de & \multicolumn{2}{|c|}{$h\left(z_{\varepsilon_{0 j}}\right)$} \\
\hline Otimização & por QG2 & por QG3 & Otimização & por QG2 & por QG3 \\
\hline Nelder-Mead & 99.99 & 95.98 & $\mathrm{CG}$ & 96.95 & 96.95 \\
\hline LBGS-B & 0.01 & 3.51 & BFGS & 3.05 & 1.25 \\
\hline BFGS & & 0.51 & NLM & & 1.80 \\
\hline Total & 100.00 & 100.00 & Total & 100.00 & 100.00 \\
\hline
\end{tabular}


Para garantir que as estimativas de $\tau_{0}$ e de $\sigma$ sejam positivas, a função $f\left(y_{i j} \mid z_{\varepsilon_{0 j}}, z_{a_{i j}}, \boldsymbol{\theta}\right)$ em (2.4) foi reescrita de modo a incorporar esta restrição. Promoveu-se uma reparametrização, tal que $\tau_{0}=\exp \left(\lambda_{\tau_{0}}\right)$ e $\sigma=\exp \left(\lambda_{\sigma}\right)$ e, posteriormente, as estimativas de $\tau_{0}$ e de $\sigma$ foram obtidas aplicando a função logarítmica, com base na propriedade de invariância dos estimadores de MV. 


\section{Capítulo 3}

\section{Resultados da simulação}

Neste capítulo serão discutidos os resultados dos ajustes realizados na simulação (identificados pelas siglas MLG, QV, QGR, QG2 e QG3, conforme descrito na Seção 2.4), considerando os casos em que:

- ambos os componentes de variância $\tau_{0}$ e $\sigma$ são nulos, de modo que os ajustes sob o Modelo 1 (MLG e QV), o Modelo 2 (QGR e QG2) e o Modelo 3 (QG3), supostamente, apresentariam resultados próximos;

- apenas $\tau_{0}$ é não nulo, caso em que apenas os ajustes sob o Modelo 1 não contemplam este componente;

- apenas $\sigma$ é não nulo, caso em que um dos ajustes sob o Modelo 1 (MLG) e os ajustes sob o Modelo 2 não incorporam este componente;

- tanto $\tau_{0}$ quanto $\sigma$ são não nulos, casos em que apenas o ajuste sob o Modelo 3 contempla ambos os componentes. 


\subsection{Vieses dos estimadores dos componentes de variância $\left(\tau_{0}\right.$ e $\left.\sigma\right)$}

Os resultados discutidos nesta seção encontram-se nas Tabelas B.1 e B.2, que contêm as médias das estimativas de $\tau_{0}$ e de $\sigma$ obtidas na simulação. Estas médias constituem estimativas do valor esperado de $\hat{\tau}_{0}$ e de $\hat{\sigma}$, respectivamente, pelos quais foram analisados os vieses dos referidos estimadores.

Daqui por diante, sempre que for dito que um estimador foi menos enviesado ou teve menor viés do que outro, deve-se interpretar que o estimador foi aquele que em módulo apresentou o menor valor esperado.

\subsection{1 $\tau_{0}$ e $\sigma$ nulos}

Quando $\tau_{0}$ e $\sigma$ são nulos, as Tabelas B.1 e B.2 mostram que as médias das estimativas de $\tau_{0}$ foram similares entre os ajustes QGR, QG2 e QG3, para o mesmo número de escolas $(J)$ e de alunos $\left(n_{j}\right)$. Para o mesmo $J$, estas médias ficaram mais próximas de zero com o aumento em $n_{j}$ e a Figura C.1 mostra, também, que as distribuições das estimativas ficaram mais concentradas. Já para o mesmo $n_{j}$, as médias pouco variaram com o aumento de $J$, assim como as distribuições das estimativas pouco se alteraram, exceto quando $\gamma_{00}=1$ no caso $n_{j}=20$, em que houve decréscimos de maior magnitude nas médias das estimativas de $J=20$ para $J=50$ e as distribuições apresentaram uma redução no $3^{\circ}$ quartil mais acentuada do que em $n_{j}=50$.

Pela Figura C.10, observa-se que houve redução dos valores do $3^{\circ}$ quartil das distribuições das estimativas de $\sigma$, tanto com o aumento em $n_{j}$ fixado $J$ quanto com o aumento em $J$ fixado $n_{j}$, o que corrobora o fato das médias das estimativas de $\sigma$, retratadas nas Tabelas B.1 e B.2, terem ficado cada vez mais próximas de zero com o aumento do tamanho da amostra.

\subsubsection{Somente $\tau_{0}$ não nulo}

Quando $\tau_{0} \neq 0$ e $\sigma$ é nulo, QGR e QG2 apresentaram os mesmos resultados quanto às médias das estimativas de $\tau_{0}$, conforme mostram as Tabelas B.1 e B.2. Com o aumento em $J$, estas médias 
ficaram mais próximas de $\tau_{0}$ e, conforme ilustram as Figuras C.2 e C.3, as estimativas de $\tau_{0}$ ficaram mais concentradas, o que é razoável já que um maior número de grupos contribui para melhorar a estimativa de um componente de variância relativo a grupo.

Para $\tau_{0}=1$, o estimador de $\tau_{0}$ em QG3 apresentou sempre o menor viés em relação a QGR e QG2 (73\% da magnitude do viés de QGR, para $\left.J=n_{j}=50\right)$. Houve redução do viés de $\hat{\tau}_{0}$ e de $\hat{\sigma}$ em QG3, tanto com o aumento em $J$ fixado $n_{j}$ quanto com o aumento em $n_{j}$ fixado $J$.

Quando $\tau_{0}=0.5$, o viés do estimador $\hat{\tau}_{0}$ em QG3 foi, de modo geral, maior do que em QGR e QG2. Somente QG3 apresentou médias das estimativas de $\tau_{0}$ superiores ao parâmetro (entre 4 e $34 \%)$.

As Figuras C.11 e C.12 mostram que a distribuição das estimativas de $\sigma$ tornou-se mais concentrada com o aumento em $J$ e/ou em $n_{j}$. De $\tau_{0}=0.5$ para $\tau_{0}=1$, as médias das estimativas de $\sigma$ em um mesmo ajuste, em geral, ficaram mais próximas de zero, assim como a mediana das estimativas de $\sigma$.

\subsubsection{Somente $\sigma$ não nulo}

Quando $\tau_{0}$ é nulo e $\sigma \neq 0$, pelas Tabelas B.1 e B.2 observa-se que, para os mesmos valores de $J$ e $n_{j}$, as médias das estimativas de $\tau_{0}$ em QGR e QG2 foram similares, porém atingiram pelo menos o triplo dos valores das correspondentes médias em QG3, cujo estimador $\hat{\tau}_{0}$ apresentou o menor viés (25 a 33\% em relação à magnitude do viés obtido por QGR/QG2). As Figuras C.4 e C.5 mostram que QGR e QG2, ao contrário de QG3, não apresentaram estimativas adequadas de $\tau_{0}$, pois estas ficaram bem acima do valor zero. Note que o $1^{\circ}$ quartil em QGR e QG2 é sempre superior ao $3^{\circ}$ quartil em QG3.

Fixado $J$, com o aumento de $n_{j}$ em QG3, tanto $\hat{\tau}_{0}$ quanto $\hat{\sigma}$ apresentaram redução em seus vieses e tenderam aos reais valores dos parâmetros. Com o aumento em $J$ para $n_{j}$ fixado, QG3 apresentou redução no viés de $\hat{\tau}_{0}$ (e também de $\hat{\sigma}$ ), enquanto que o oposto ocorreu em QGR e QG2. Quando $n_{j}=50$, os vieses em QGR e QG2 são menores do que quando $n_{j}=20$. Portanto, a melhora das médias das estimativas de $\tau_{0}$ em QGR e QG2 foi determinada pelo aumento em $n_{j}$ e não pelo aumento em $J$. Como QGR e QG2 não contemplam a variabilidade imposta por 
$\sigma$, esta parece ter sido confundida com a variabilidade entre grupos e ter feito com que $\tau_{0}$ fosse inflacionado.

As Figuras C.13 e C.14 apresentam estimativas de $\sigma$ distribuídas de forma aproximadamente simétrica, em torno do seu real valor. Além disso, elas ficam cada vez mais concentradas com o aumento do tamanho da amostra.

\subsection{4 $\tau_{0}$ e $\sigma$ não nulos}

Considerando somente os casos em que tanto $\tau_{0}$ quanto $\sigma$ não são nulos, nota-se pelas Figuras C.6 a C.9 que embora os ajustes que utilizam QGHA, avaliados sob os mesmos $J$ e $n_{j}$, tenham apresentado boxplots com intervalos interquartis e amplitudes semelhantes para as estimativas de $\tau_{0}$, os valores do $1^{\circ}$ e do $3^{\circ}$ quartis em QG3 são superiores em relação a QGR e QG2. Embora as distribuições tenham se mostrado aproximadamente simétricas nos três ajustes, esta simetria não se deu em torno de $\tau_{0}$ em nenhum deles. Quando $\sigma=0.5$, observa-se que o valor de $\tau_{0}$ ficou sempre entre a mediana e o $3^{\circ}$ quartil nos ajustes QGR e QG2, sendo que em QG3 a mediana ficou mais próxima de $\tau_{0}$ do que nos outros dois ajustes na maioria dos casos. Quando $\sigma=1$, QGR e QG2 apresentaram o $3^{\circ}$ quartil sempre abaixo de $\tau_{0}$, enquanto QG3 tem suas estimativas de $\tau_{0}$ distribuídas de modo que $\tau_{0}$ ficou sempre mais próximo da mediana do que $3^{\circ}$ quartil.

As Tabelas B.1 e B.2 mostram que para $\tau_{0}=1$, sob os mesmos valores de $\sigma, J$ e $n_{j}$, QGR e QG2 exibiram praticamente as mesmas estimativas do valor esperado de $\hat{\tau}_{0}$. Estas foram sempre inferiores ao valor de $\tau_{0}$, assim como as correspondentes estimativas em QG3. No entanto, neste último, $\hat{\tau}_{0}$ mostrou vieses de menor magnitude em relação a QGR/QG2 (9 a 27\% quando $\sigma=0.5$ e 24 a $54 \%$ quando $\sigma=1$ ), especialmente quando $J=50$. . Fixando-se $n_{j}$, o estimador $\hat{\tau}_{0}$ apresentou redução de seu viés com o aumento de $J$, nos três ajustes. Em QGR e QG2, quando $\sigma$ passou de 0.5 para 1 unidade, as médias das estimativas de $\tau_{0}$ ficaram mais distantes do valor 1 em relação às respectivas médias em QG3, já que QGR e QG2 não contemplam $\sigma$. Além disso, para o mesmo valor de $J$, com o aumento em $n_{j}$, as médias das estimativas também ficaram mais longe de 1 e isto, de modo geral, foi mais acentuado quando $\sigma$ passou de 0.5 para 1 . Ou seja, predominantemente, quanto maior foi o valor de $\sigma$, mais distantes de $\tau_{0}$ ficaram as médias de suas estimativas obtidas em QGR e QG2 e isto se agravou com o aumento em $n_{j}$. 
Para $\tau_{0}=0.5$, QGR e QG2 novamente produziram as mesmas estimativas do valor esperado de $\hat{\tau}_{0}$, sob os mesmos valores de $J$ e $n_{j}$, as quais também foram inferiores ao valor de $\tau_{0}$. Em QG3 $\hat{\tau}_{0}$ só não apresentou menor viés em relação a QGR e QG2 para $\sigma=0.5$ e $J=n_{j}=50$, caso em

que o valor esperado do estimador superou o valor do parâmetro. Novamente, quanto maior foi o valor de $\sigma$, mais prejudicadas ficaram as médias das estimativas de $\tau_{0}$ obtidas em QGR e QG2 e isto se agravou com o aumento de $n_{j}$.

As Tabelas B.1 e B.2 mostram que o viés do estimador de $\sigma$ tendeu a zero sempre que $\tau_{0}$ e $\sigma$ são ambos não nulos. As Figuras C.15 a C.18 mostram que a distribuição das estimativas de $\sigma$ foi aproximadamente simétrica em torno do seu real valor. Além disso elas ficaram cada vez mais concentradas com o aumento do tamanho da amostra.

\subsection{Resultados para $\gamma_{00}$}

\subsubsection{Avaliação do estimador $\hat{\gamma}_{00}$}

As Tabelas B.3 e B.4 trazem as estimativas do valor esperado de $\hat{\gamma}_{00}$ sob cada ajuste, ou seja, as estimativas do viés de $\hat{\gamma}_{00}$, fixados os valores de $\gamma_{00}, \tau_{0}$ e $\sigma$. As Figuras D.1 a D.10 ilustram os boxplots das estimativas de $\gamma_{00}$.

As Tabelas B.5 e B.6 trazem as médias das estimativas do EP de $\hat{\gamma}_{00}$ sob cada ajuste e as Figuras D.11 a D.19 trazem os boxplots. O EP foi estimado para cada réplica da simulação, por meio da matriz de informação observada, conforme descrito na Seção 2.4. As Tabelas B.7 e B.8 trazem os DPs para as estimativas de $\gamma_{00}$ sob cada ajuste.

$\mathrm{O}$ valor da média das estimativas do EP de $\hat{\gamma}_{00}$ apresentada nas Tabelas B.5 e B.6, fixados os mesmos parâmetros e tamanhos de amostra $J$ e $n_{j}$, foi similar ao valor do DP das estimativas de $\gamma_{00}$ das respectivas Tabelas B.7 e B.8 para QGR, QG2 e QG3. Quando $\tau_{0}=\sigma=0$, MLG e QV apresentaram DPs próximos aos respectivos EPs. Para $\tau_{0}$ ou $\sigma$ não nulo, MLG mostrou sempre DPs bem superiores aos EPs, enquanto QV apresentou EPs e DPs semelhantes somente nos casos em que apenas o desvio padrão $\sigma$ era não nulo. 
Quando $\gamma_{00}=0$, os vieses estimados para $\hat{\gamma}_{00}$ de modo geral tenderam a zero em todos os ajustes (veja Tabela B.3).

Quando $\gamma_{00}=1$ e $\tau_{0}=\sigma=0$ (veja Tabela B.4), os vieses estimados para $\hat{\gamma}_{00}$ tenderam a zero. No entanto, sob os mesmos valores de $J$ e $n_{j}$, os vieses em QG3 excederam ligeiramente (entre 0.00058 e 0.00144 unidade) os correspondentes valores em QGR e QG2.

Vê-se pelas Figuras D.1 e D.2 que as estimativas de $\gamma_{00}$ também se distribuíram de forma similar entre os ajustes e que elas ficaram mais concentradas em torno do valor de $\gamma_{00}$ à medida que $J$ e $n_{j}$ aumentaram.

Quando $\gamma_{00}=0, \tau_{0} \neq 0$ e $\sigma=0$, a magnitude do viés (Tabela B.3) e a distribuição das estimativas (Figura D.3) foram similares entre os ajustes, sob os mesmos valores de $\tau_{0}, J$ e $n_{j}$. As estimativas de $\gamma_{00}$ ficaram menos dispersas quando $\tau_{0}=0.5$, em relação a $\tau_{0}=1$ e, também, perante um maior número de grupos $(J=50)$.

Quando $\gamma_{00}=1, \tau_{0} \neq 0$ e $\sigma=0$, o estimador mais enviesado ocorreu no MLG/QV (Tabela B.4), cujas estimativas estão concentradas abaixo do valor 1 (veja Figura D.4), diferentemente dos demais ajustes, em que elas concentraram-se em torno de 1. Vê-se, também, que as estimativas de $\gamma_{00}$ quando $J=50$ ficaram mais concentradas, em todos os ajustes. QGR e QG2 apresentaram os menores vieses para $\hat{\gamma}_{00}$. Os vieses estimados de $\hat{\gamma}_{00}$ em QG3 quase se equipararam aos respectivos vieses obtidos em QGR e QG2 com o aumento em $\tau_{0}$ de 0.5 para 1, enquanto o viés de $\hat{\gamma}_{00}$ se acentuou no MLG/QV.

Quando $\gamma_{00}=0, \tau_{0}=0$ e $\sigma \neq 0$, a magnitude do viés foi similar entre os ajustes, para os mesmos valores fixados de $\tau_{0}, J$ e $n_{j}$ (veja Tabela B.4). A Figura D.5 ilustra estimativas de $\gamma_{00}$ mais concentradas quando houve um maior número de grupos $(J=50)$ e um maior número de unidades por grupo $\left(n_{j}=50\right)$. Além disso, quando $\sigma=0.5$ todos os ajustes para os mesmos $J$ e $n_{j}$ resultaram em estimativas com distribuições similares. Já em $\sigma=1$, além das distribuições terem ficado mais dispersas do que em $\sigma=0.5$, QG3 teve estimativas mais espalhadas do que nos demais ajustes avaliados nas mesmas condições.

Quando $\gamma_{00}=1, \tau_{0}=0$ e $\sigma \neq 0$, o viés do estimador $\hat{\gamma}_{00}$ tendeu a zero somente em QG3 (veja Tabela B.4). Os demais ajustes apresentaram vieses de magnitudes similares para os mesmos 
$J$ e $n_{j}$, que se acentuaram com o aumento de $\sigma$. Desta forma QG3 foi o único ajuste a apresentar estimativas de $\gamma_{00}$ em torno do valor 1 (veja Figura D.6).

Quando $\tau_{0}=0$ e $\sigma \neq 0$, as Figuras D.14 e D.15 mostraram que os EPs estimados de $\hat{\gamma}_{00}$ em QG3 foram em geral maiores do que nos demais ajustes, porém tiveram menor variabilidade do que QGR e QG2. Além disso, embora os vieses tenham apresentado comportamentos diferentes quando $\gamma_{00}=0$ e quando $\gamma_{00}=1$, não houve diferença com relação ao quadro de distribuição dos EPs de $\hat{\gamma}_{00}$.

Quando $\gamma_{00}=0, \tau_{0}=0.5$ e $\sigma \neq 0$, a magnitude do viés (B.3) e a distribuição das estimativas de $\gamma_{00}$ (veja Figura D.7) foram similares entre os ajustes, para os mesmos valores fixados de $\sigma, J$ e $n_{j}$. A distribuição das estimativas em um mesmo ajuste pouco se alterou quando $\sigma$ aumentou para 1, com exceção de QG3, que apresentou estimativas um pouco mais dispersas do que no caso em que $\sigma=0.5$. A Figura D.7 mostrou também distribuições mais concentradas em torno de 0 quando $J=50$.

Quando $\gamma_{00}=0, \tau_{0}=1$ e $\sigma \neq 0$, a magnitude do viés foi similar entre os ajustes, para os mesmos valores fixados de $\sigma, J$ e $n_{j}$ (veja Tabela B.3). A distribuição das estimativas variou mais do MLG/QV para QGR/QG2 e destes para QG3, como mostra a Figura D.9, em que o $1^{\circ}$ e o $3^{\circ}$ quartis, em todos os ajustes, estão inseridos no intervalo interquartil obtido em QG3, sob os mesmos valores de $J$ e $n_{j}$.

Quando $\gamma_{00}=1, \tau_{0} \neq 0$ e $\sigma \neq 0$ (veja Tabela B.4), sob os mesmos valores de $\tau_{0}, \sigma, J$ e $n_{j}$, o viés do estimador $\hat{\gamma}_{00}$ tendeu a zero somente em QG3, os ajustes QGR e QG2 apresentaram vieses de magnitudes similares e MLG/QV teve o maior viés. O aumento de $\sigma$ de 0.5 para 1 , sob os mesmos valores de $\tau_{0}, J$ e $n_{j}$, implicou no aumento dos vieses e, também, no deslocamento das estimativas, afastando-se do valor 1 (veja Figuras D.8 e D.10), exceto em QG3. Desta forma, QG3 foi o único ajuste a apresentar estimativas apropriadas de $\gamma_{00}$, concentradas em torno do valor 1 .

As Figuras D.16 a D.19, em que $\tau_{0} \neq 0$ e $\sigma \neq 0$, mostram que as estimativas do EP de $\hat{\gamma}_{00}$ em QG3, quando $\sigma$ passa de 0.5 para 1 , concentraram-se ainda mais acima das estimativas dos demais ajustes. Os boxplots dos EPs estimados de $\hat{\gamma}_{00}$ não diferiram entre os casos $\gamma_{00}=0$ e $\gamma_{00}=1$, embora estes tenham apresentado vieses com comportamentos diferentes. 
É importante ressaltar que o parâmetro $\gamma_{00}$ tem diferentes interpretações nos Modelos 1,2 e 3. O Modelo 1 é chamado de marginal ou population-average. Já os Modelos 2 e 3 são chamados de condicionais, subject-specific ou unit-specific, ou ainda, cluster-specific.

É conveniente designar, neste momento, o parâmetro $\gamma_{00}$ nos Modelos 1,2 e 3 por $\gamma_{00}{ }^{(1)}$, $\gamma_{00}{ }^{(2)}$ e $\gamma_{00}{ }^{(3)}$, respectivamente. No Modelo 1 foi suposto que a resposta de cada aluno de cada escola segue uma distribuição binomial, em que $\log \left(\frac{p_{i j}}{1-p_{i j}}\right)$ foi assumido constante para todos os indivíduos $\left(=\gamma_{00}{ }^{(1)}\right)$. Logo, $\left(1+e^{-\gamma_{00}{ }^{(1)}}\right)^{-1}$ é a probabilidade média de acerto para todos os alunos, de todas as escolas. Já no Modelo 2, admitiu-se que, condicionalmente ao efeito aleatório $\varepsilon_{0 j}$, a resposta de cada aluno dentro desta escola tem uma distribuição binomial, em que $\log \left(\frac{p_{i j}}{1-p_{i j}}\right)$ foi considerado constante para todos os indivíduos da mesma escola $\left(=\gamma_{00}{ }^{(2)}+\varepsilon_{0 j}\right)$. Portanto, $\left(1+e^{-\gamma_{00}{ }^{(2)}}\right)^{-1}$ é a probabilidade de acerto para todos os alunos, de uma escola mediana ou "típica" (i.e., que $\varepsilon_{0 j}=0$ ). Analogamente, no Modelo 3, foi assumido que a resposta de cada aluno, condicionalmente a $\varepsilon_{0 j}$ e $a_{i j}$, possui uma distribuição binomial, em que $\log \left(\frac{p_{i j}}{1-p_{i j}}\right)$ varia conforme a escola e o aluno $\left(=\gamma_{00}{ }^{(3)}+\varepsilon_{0 j}+a_{i j}\right)$. Desta forma, $\left(1+e^{-\gamma_{00}{ }^{(3)}}\right)^{-1}$ é a probabilidade de acerto para um aluno mediano, de uma escola "típica" (i.e., que $\varepsilon_{0 j}=a_{i j}=0$ ).

Para $\tau_{0}=\sigma=0$, tem-se que $\gamma_{00}{ }^{(1)}=\gamma_{00}{ }^{(2)}=\gamma_{00}{ }^{(3)}$. Quando somente $\tau_{0}=0, \gamma_{00}{ }^{(1)}=\gamma_{00}{ }^{(2)}$. Quando somente $\sigma=0, \gamma_{00}{ }^{(2)}=\gamma_{00}{ }^{(3)}$. A discrepância entre $\gamma_{00}{ }^{(1)}$ e $\gamma_{00}{ }^{(2)}$ aumenta com $\tau_{0}$. Já entre $\gamma_{00}{ }^{(2)}$ e $\gamma_{00}{ }^{(3)}$, a diferença cresce com $\sigma$.

Quando $\gamma_{00}$ assume o valor 0, a f.d.p. de $p_{i j}$ é simétrica em torno de 0.5, como mostra a Figura 2.1. Já para $\gamma_{00}=1$, a f.d.p. de $p_{i j}$ é assimétrica. De modo geral, a assimetria da curva logística-normal acentua-se à medida que $\gamma_{00}$ afasta-se do valor 0 e, também, com o aumento de $\tau_{0}^{2}+\sigma^{2}$

Quando $\gamma_{00}=0$, em decorrência da simetria da curva logística-normal, a probabilidade de acerto de um aluno de uma escola mediana e a probabilidade de acerto de um aluno mediano de uma escola mediana, coincidem com a probabilidade média de acerto na população de alunos em geral. Isto explica a proximidade dos vieses estimados para $\hat{\gamma}_{00}$ em todos os ajustes feitos sob a mesma combinação dos parâmetros, conforme mostra a Tabela B.3.

Por outro lado, quando $\gamma_{00} \neq 0$, a assimetria da f.d.p. de $p_{i j}$ faz com que os parâmetros 
$\gamma_{00}{ }^{(1)}, \gamma_{00}{ }^{(2)}$ e $\gamma_{00}{ }^{(3)}$ tenham as diferentes interpretações anteriormente colocadas, pois

$$
\mathrm{E}_{a_{i j}}\left[\left(1+e^{-\gamma_{00}{ }^{(3)}-\varepsilon_{0 j}-a_{i j}}\right)^{-1}\right]=\left(1+e^{-\gamma_{00}{ }^{(2)}-\varepsilon_{0 j}}\right)^{-1} \neq\left(1+e^{-\gamma_{00}^{(3)}-\varepsilon_{0 j}}\right)^{-1}
$$

$\mathrm{e}$

$$
\mathrm{E}_{\varepsilon_{0 j}}\left[\left(1+e^{-\gamma_{00}{ }^{(2)}-\varepsilon_{0 j}}\right)^{-1}\right]=\left(1+e^{-\gamma_{00}{ }^{(1)}}\right)^{-1} \neq\left(1+e^{-\gamma_{00}^{(2)}}\right)^{-1}
$$

Neste caso os vieses referem-se a diferentes estimadores, salvo as exceções anteriormente mencionadas em que a nulidade de $\tau_{0}$ e/ou de $\sigma$ promove a coincidência de $\gamma_{00}$ entre alguns modelos.

Por exemplo, quando $\tau_{0}=0$ e $\sigma=1$, tem-se que $\gamma_{00}{ }^{(1)}=\gamma_{00}{ }^{(2)} \neq \gamma_{00}{ }^{(3)}$. Pela Tabela 2.1, $\mathrm{E}\left(p_{i j}\right) \cong 0.697$, de modo que $\log \left(\frac{0.697}{1-0.697}\right)=0.832$, explicando o motivo dos vieses em MLG/QV, QGR e QG2 estarem próximos de -0.168 na Tabela B.4.

As diferenças entre $\gamma_{00}{ }^{(1)}, \gamma_{00}{ }^{(2)}$ e $\gamma_{00}{ }^{(3)}$ continuam a existir para outras funções de ligação não-lineares, embora com outras magnitudes, mas desaparecem ao utilizar a função de ligação identidade. Veja mais sobre o assunto em Raudenbush e Bryk (2002), Diggle, Heagerty, Liang e Zeger (2002) e Molenberghs e Verbeke (2005).

Quando $\gamma_{00}=0$, nota-se ao contrastar dois conjuntos de valores para os componentes de variância, $\left(\tau^{(1)}, \sigma^{(1)}\right)$ e $\left(\tau_{0}^{(2)}, \sigma^{(2)}\right)$, em que $\tau_{0}^{(1)}=\sigma^{(2)}$ e $\tau_{0}^{(2)}=\sigma^{(1)}$, que o conjunto em que $\tau_{0}>\sigma$ apresenta estimativas para $\gamma_{00}$ muito mais dispersas em torno de zero do que o conjunto em que $\tau_{0}<\sigma$. Embora a variância induzida em $p_{i j}$ seja a mesma nas duas situações (veja Tabela 2.1), a dependência entre os alunos que estudam na mesma escola é maior no caso em que $\tau_{0}>\sigma$, o que explica porque os EPs estimados de $\hat{\gamma_{0}}$ foram maiores do que quando $\tau_{0}<\sigma$.

\subsubsection{Avaliação da taxa de rejeição para $\gamma_{00}$}

A partir das estimativas de $\gamma_{00}$ e do EP estimado para $\hat{\gamma}_{00}$ em cada amostra, foram construídos intervalos de $95 \%$ de confiança para $\gamma_{00}$. Avaliou-se quantas amostras sob a mesma configuração não continham o valor previamente estabelecido para $\gamma_{00}$ no intervalo de confiança construído, estabelecendo-se a taxa de rejeição estimada do teste de hipóteses $\mathrm{H}_{0}: \gamma_{00}=\gamma_{00}{ }^{*}$ vs $\mathrm{H}_{\mathrm{a}}: \gamma_{00} \neq$ $\gamma_{00}{ }^{*}$ pela estatística de Wald, em que $\gamma_{00}{ }^{*}=0,1$ (veja Tabelas B.9 e B.10). Caso o estimador 
$\hat{\gamma}_{00}$ satisfaça certas condições de regularidade e os tamanhos de amostra sejam suficientemente grandes, espera-se que, aproximadamente, $5 \%$ das amostras simuladas sob a mesma configuração não contenham o verdadeiro valor de $\gamma_{00}$.

As taxas de rejeição estimadas podem ser encontradas nas Tabelas B.9 e B.10, para $\gamma_{00}=0$ e $\gamma_{00}=1$, respectivamente.

As taxas de rejeição empíricas nos casos em que $\tau_{0}=\sigma=0$ ficaram todas em torno de $5 \%$ (entre $3.1 \%$ e $6.1 \%$ ), uma vez que na ausência dos componentes $\tau_{0}$ e $\sigma$, o ajuste de um MLG equivale a uma forma reduzida dos ajustes QV, QGR, QG2 e QG3.

Quando $\tau_{0} \neq 0$ e $\sigma=0$, somente QGR, QG2 e QG3 mostraram taxas não muito distantes de $5 \%$. As taxas produzidas no MLG e em QV foram todas superiores a 56\%. QGR apresentou taxas melhores (mais perto de 5\%) que em QG2 e QG3. Todas as taxas em QG2 foram maiores ou iguais às de QGR e metade delas menores ou iguais às de QG3. Observa-se que com exceção de quando $\gamma_{00}=1$ e $\tau_{0}=0.5$, os ajustes que envolvem a QGHA apresentaram as taxas mais próximas de $5 \%$ quando se utilizou um maior número de grupos $(J=50)$.

Para $\tau_{0}=0$ e $\sigma \neq 0$ quando $\gamma_{00}=0$, o único ajuste que não apresentou taxas em torno de $5 \%$ foi o MLG. Já quando $\gamma_{00}=1$, as taxas obtidas em QG3 foram as únicas que se aproximaram de $5 \%$.

Para $\tau_{0} \neq 0$ e $\sigma \neq 0$ quando $\gamma_{00}=0$, somente os ajustes QGR, QG2 e QG3 apresentaram taxas estimadas em torno de $5 \%$ e que pouco variaram entre estes ajustes. Quando $\gamma_{00}=1 \mathrm{e}$ $\tau_{0}=\sigma=0.5$, QG3 apresentou melhores taxas estimadas que QGR e QG2, em especial para $J=50$. Com o aumento em $\sigma$ de 0.5 para 1, apenas QG3 mostrou taxas de rejeição empíricas apropriadas. Para $\tau_{0}=1$ e $\sigma=0.5$, QG3 apresentou as menores taxas estimadas, exceto em $J=n_{j}=20$. Novamente observa-se que com o aumento em $\sigma$ de 0.5 para 1 , QG3 tornou-se o único ajuste que apresentou estimativas apropriadas das taxas de rejeição.

De acordo com comentários da seção anterior, as médias das estimativas dos EPs de $\hat{\gamma}_{00}$ foram sempre próximas dos desvios padrões das estimativas de $\gamma_{00}$ tanto para $\gamma_{00}=0$ quanto para $\gamma_{00}=1$ nos ajustes QGR, QG2 e QG3. Mas as taxas de rejeição empíricas de QGR e QG2 ficaram próximas às de QG3 em $\gamma_{00}=0$, enquanto que em $\gamma_{00}=1$ em geral isto não ocorreu quando $\sigma \neq 0$, 
que é justamente a única situação em que $\gamma_{00}$ representa diferentes entidades nos Modelos 2 e 3. 



\section{Capítulo 4}

\section{Aplicação}

Este capítulo traz uma aplicação na área de educação, realizada em Londrina-PR (veja Poli (2000)). Alunos da $1^{\mathrm{a}}$ série do ensino fundamental de 8 diferentes escolas $(J=8)$ foram submetidos a uma avaliação composta por 20 itens $(K=20)$. Utilizando como variável resposta o número de acertos de cada aluno, avaliou-se este escore como uma medida de desempenho. O número de alunos por escola, $n_{j}$, foi no mínimo 43 e no máximo 101, resultando em $\sum_{j=1}^{J} n_{j}=568$ alunos. Os dados foram avaliados pelos ajustes MLG, QV, QGR, QG2 e QG3 e os resultados estão na Tabela 4.1.

A Tabela 4.1 mostra que as estimativas pontuais de $\gamma_{00}$ foram similares, porém quando se passou do ajuste do MLG para QV, de QV para QGR/QG2 e destes últimos para QG3 houve um aumento da variância do estimador $\hat{\gamma}_{00}$, resultando em intervalos de confiança (ICs) de $95 \%$ para $\left(1+e^{-\gamma_{00}}\right)^{-1}$ com amplitudes maiores, como já era esperado. É importante ressaltar as diferentes interpretações da quantidade $\left(1+e^{-\gamma_{00}}\right)^{-1}$ nos diferentes ajustes. No MLG e em QV, $\left(1+e^{-\gamma_{00}}\right)^{-1}$ representa a probabilidade média de acerto na população de alunos em geral. Já em QGR/QG2, retrata a probabilidade de acerto de um aluno de uma escola mediana (dado $\left.\varepsilon_{0 j}=0\right)$ e em QG3 corresponde à probabilidade de acerto de um aluno mediano de uma escola mediana $\left(\right.$ dados $\left.\varepsilon_{0 j}=a_{i j}=0\right)$.

O ajuste QG3 mostrou menor correlação intraclasse em relação a QGR/QG2, sendo que a estimativa do componente $\sigma$ apresentou maior magnitude do que a de $\tau_{0}$, ou seja, a dependência 
Tabela 4.1: Resultados da aplicação.

\begin{tabular}{|c|c|c|c|c|c|}
\hline Quantidades & MLG & $\mathrm{QV}$ & QGR & QG2 & QG3 \\
\hline$\left(1+e^{-\hat{\gamma}_{00}}\right)^{-1}$ & 0.5216 & 0.5216 & 0.5180 & 0.5177 & 0.5216 \\
\hline IC de $95 \%$ & {$[0.5124 ; 0.5307]$} & {$[0.5027 ; 0.5404]$} & {$[0.4364 ; 0.5986]$} & {$[0.4395 ; 0.5951]$} & {$[0.4316 ; 0.6101]$} \\
\hline$\hat{\gamma}_{00}\left(\mathrm{EP}\left[\hat{\gamma}_{00}\right]\right)$ & $0.0863(0.0188)$ & $0.0863(0.0386)$ & $0.0719(0.1671)$ & $0.0710(0.1602)$ & $0.0863(0.1845)$ \\
\hline$\hat{\tau}_{0}\left(\operatorname{EP}\left[\hat{\tau}_{0}\right]\right)$ & & & $0.4492(0.1212)$ & $0.4497(0.1142)$ & $0.5079(0.1332)$ \\
\hline$\hat{\sigma}(\operatorname{EP}[\hat{\sigma}])$ & & & & & $0.8330(0.0371)$ \\
\hline$\hat{\phi}$ & & 4.2270 & & & \\
\hline$\overline{\operatorname{Corr}}\left[\hat{\gamma}_{00}, \hat{\tau}_{0}\right]$ & & & -0.2957 & -0.0018 & -0.0023 \\
\hline $\operatorname{Corr}\left[\hat{\gamma}_{00}, \hat{\sigma}\right]$ & & & & & 0.0042 \\
\hline $\operatorname{Corr}\left[\hat{\tau}_{0}, \hat{\sigma}\right]$ & & & & & 0.0183 \\
\hline Corr. intraclasse & & & 0.5148 & 0.5154 & 0.2399 \\
\hline Desviância & 2689.37 & & 2125.42 & 2125.42 & 1388.73 \\
\hline GL & 567 & & 566 & 566 & 565 \\
\hline Log-verossim. * & -2241.11 & & -1959.14 & -1959.14 & -1590.79 \\
\hline
\end{tabular}

${ }^{*} \mathrm{O}$ valor para o modelo saturado é -896.43

entre alunos da mesma escola é menor do que entre as respostas aos itens dadas pelo mesmo aluno. As desviâncias apresentaram magnitudes bem superiores aos graus de liberdade (GL), indicando que mesmo que a distribuição qui-quadrado não seja apropriada, parece haver superdispersão no MLG, que se mantém em QGR, QG2 e QG3, embora neste último ela tenha sido a menor.

Com o objetivo de avaliar a qualidade do ajuste dos modelos 1, 2 e 3, foram construídos envelopes simulados sob os respectivos ajustes MLG, QG2 e QG3. Para o MLG, a construção do envelope simulado seguiu a abordagem usual (veja Paula (2004), por exemplo). Para o MLGH binomial-normal descrito pelos modelos 2 e 3, a definição de seus resíduos condicionais foi inspirada em Nobre (2004), que discutiu o uso de resíduos condicionais para modelos lineares mistos. Os resíduos condicionais obtidos sob os ajustes QG2 e QG3 foram então avaliados por meio dos componentes da função de desviância e também pelo resíduo de Pearson, cujas estimativas foram obtidas respectivamente por

$$
\hat{r}_{D_{i j}}=\frac{\operatorname{sinal}\left\{y_{i j}-\mathrm{E}\left[Y_{i j} \mid \hat{\varepsilon}_{0 j}, \hat{a}_{i j}, \hat{\boldsymbol{\theta}}\right]\right\}}{\left\{2 \log f\left(y_{i j} \mid p_{i j}=\frac{y_{i j}}{K}\right)-\log f\left(y_{i j} \mid p_{i j}=\mathrm{E}\left[p_{i j} \mid \hat{\varepsilon}_{0 j}, \hat{a}_{i j}, \hat{\boldsymbol{\theta}}\right]\right)\right\}^{1 / 2}}
$$




$$
\hat{r}_{P_{i j}}=\frac{y_{i j}-\mathrm{E}\left[Y_{i j} \mid \hat{\varepsilon}_{0 j}, \hat{a}_{i j}, \hat{\boldsymbol{\theta}}\right]}{\left\{\operatorname{Var}\left[Y_{i j} \mid \hat{\varepsilon}_{0 j}, \hat{a}_{i j}, \hat{\boldsymbol{\theta}}\right]\right\}^{1 / 2}}
$$

em que $\hat{\varepsilon}_{0 j}$ e $\hat{a}_{i j}$ representam as modas a posteriori dos efeitos aleatórios e $\hat{\boldsymbol{\theta}}$ são os estimadores de MV de $\boldsymbol{\theta}=\left(\gamma_{00}, \tau_{0}, \sigma\right)$. A utilização das modas a posteriori dos efeitos aleatórios em vez de suas médias a posteriori, como sugere por exemplo Fahrmeir e Tutz (2001), é vantajosa por não requerer integração e por elas serem facilmente obtidas maximizando uma quantidade que envolve apenas a distribuição condicional de $Y_{i j}$ e as distribuições marginais de $\varepsilon_{0 j}$ e $a_{i j}$, ou seja:

$$
\hat{\varepsilon}_{0 j}=\underset{\varepsilon_{0 j}}{\arg \max } \prod_{i=1}^{n_{j}} f\left(y_{i j} \mid \varepsilon_{0 j}, \hat{\boldsymbol{\theta}}\right) f\left(\varepsilon_{0 j} \mid \hat{\tau}_{0}\right)
$$

$\mathrm{e}$

$$
\left(\hat{\varepsilon}_{0 j}, \hat{a}_{1 j}, \ldots, \hat{a}_{n_{j} j}\right)=\underset{\left(\varepsilon_{0 j}, a_{1 j}, \ldots, a_{n_{j} j}\right)}{\arg \max } \prod_{i=1}^{n_{j}} f\left(y_{i j} \mid \varepsilon_{0 j}, a_{i j}, \hat{\boldsymbol{\theta}}\right) f\left(a_{i j} \mid \hat{\sigma}\right) f\left(\varepsilon_{0 j} \mid \hat{\tau}_{0}\right)
$$

sob os ajustes QG2 e QG3 respectivamente.

Na construção dos envelopes para os ajustes QG2 e QG3, foram geradas 1367 amostras de 568 observações $\operatorname{sob} \boldsymbol{\theta}=\hat{\boldsymbol{\theta}}$. A partir de cada amostra foram estimados os efeitos aleatórios e obtidos os resíduos condicionais estimados tanto por (4.1) quanto por (4.2). Ordenaram-se os resíduos dentro de cada amostra e determiaram-se os quantis 0.005 e 0.995 para as estimativas de suas estatísticas de ordem, formando uma banda de confiança de $99 \%$.

As Figuras 4.1, 4.2 e 4.3 apresentam os envelopes para os ajustes MLG, QG2 e QG3 respectivamente, indicando que apenas em QG3 não há indícios de que o modelo não esteja adequadamente ajustado. A Figura 4.4-(a) traz os boxplots das probabilidades observadas para cada escola. Já a Figura 4.4-(b) mostra os boxplots das probabilidades estimadas para cada escola pelo ajuste QG3, juntamente com as estimativas para cada escola sob o ajuste QG2, representadas pelo símbolo $\square$, e a estimativa sob o ajuste do MLG, dada pela linha tracejada. Observa-se que as medianas das probabilidades observadas ficaram bem próximas das medianas das probabilidades estimadas por QG3 enquanto que QG2 apresentou estimativas um pouco mais afastadas. 

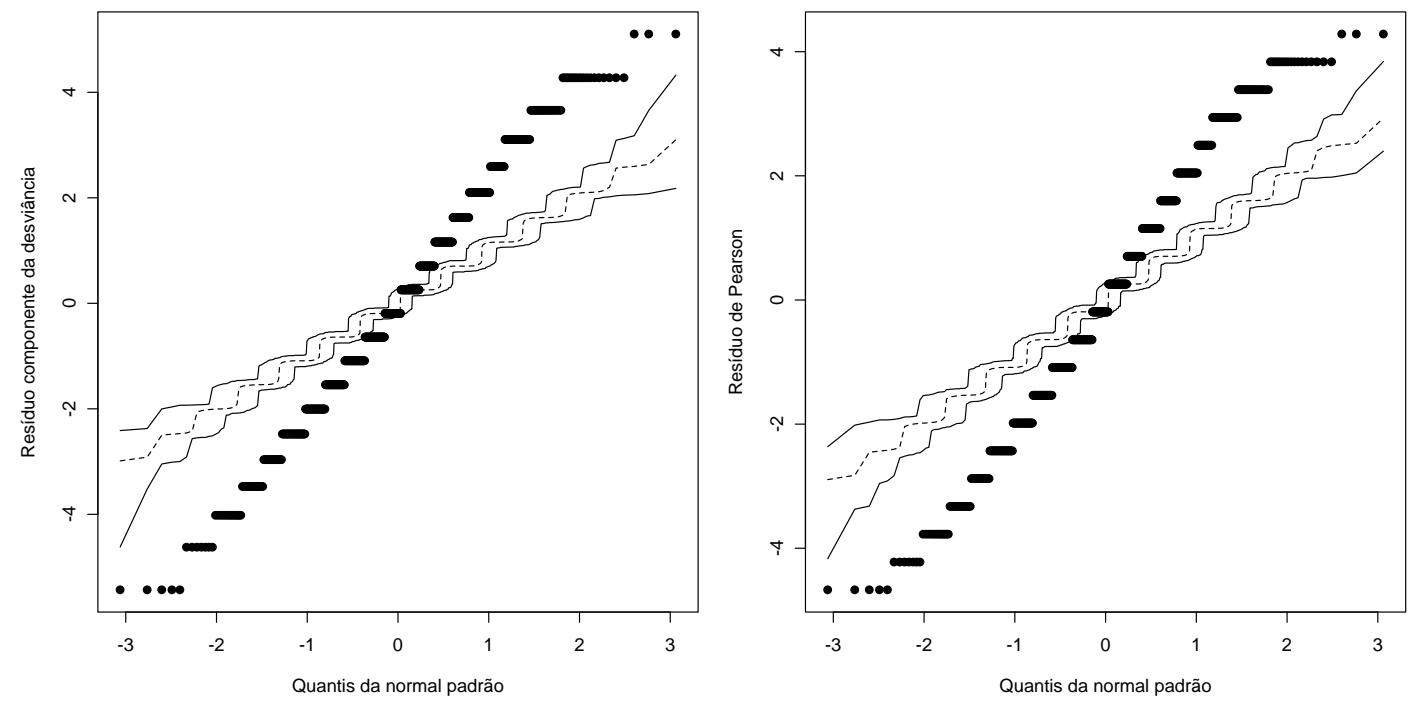

Figura 4.1: Envelopes para o ajuste do MLG com 99 \% de confiança
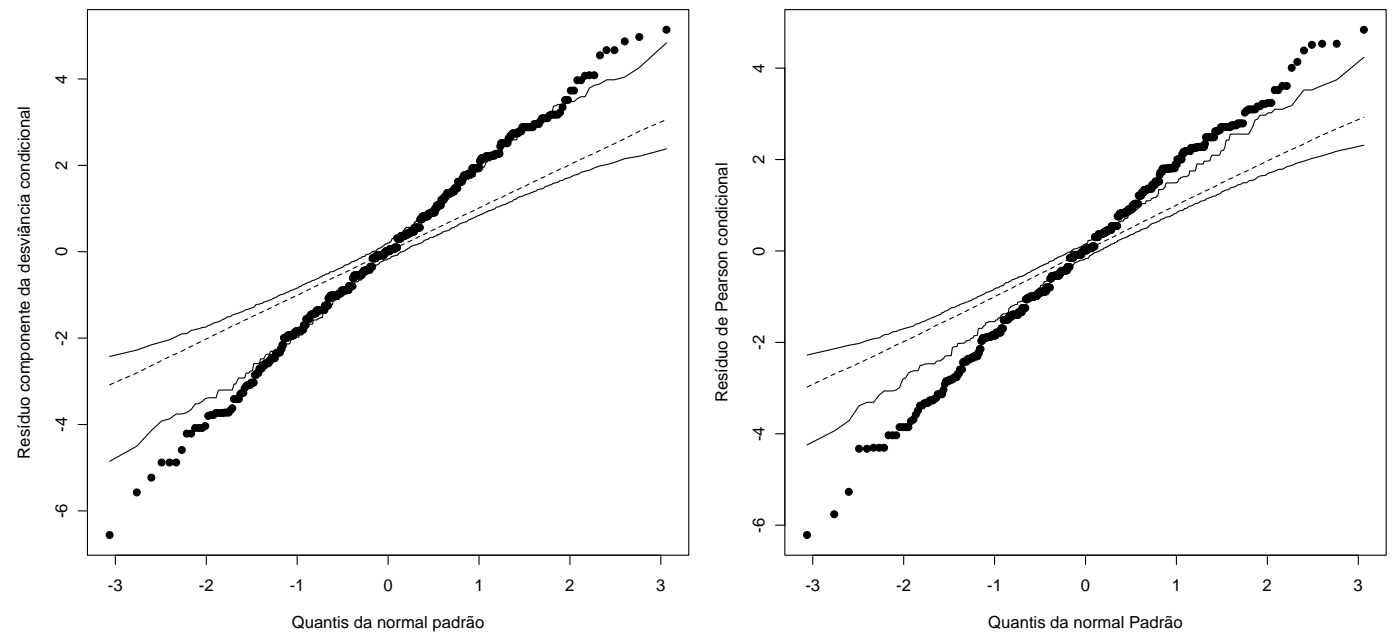

Figura 4.2: Envelopes para o ajuste de QG2 com $99 \%$ de confiança 

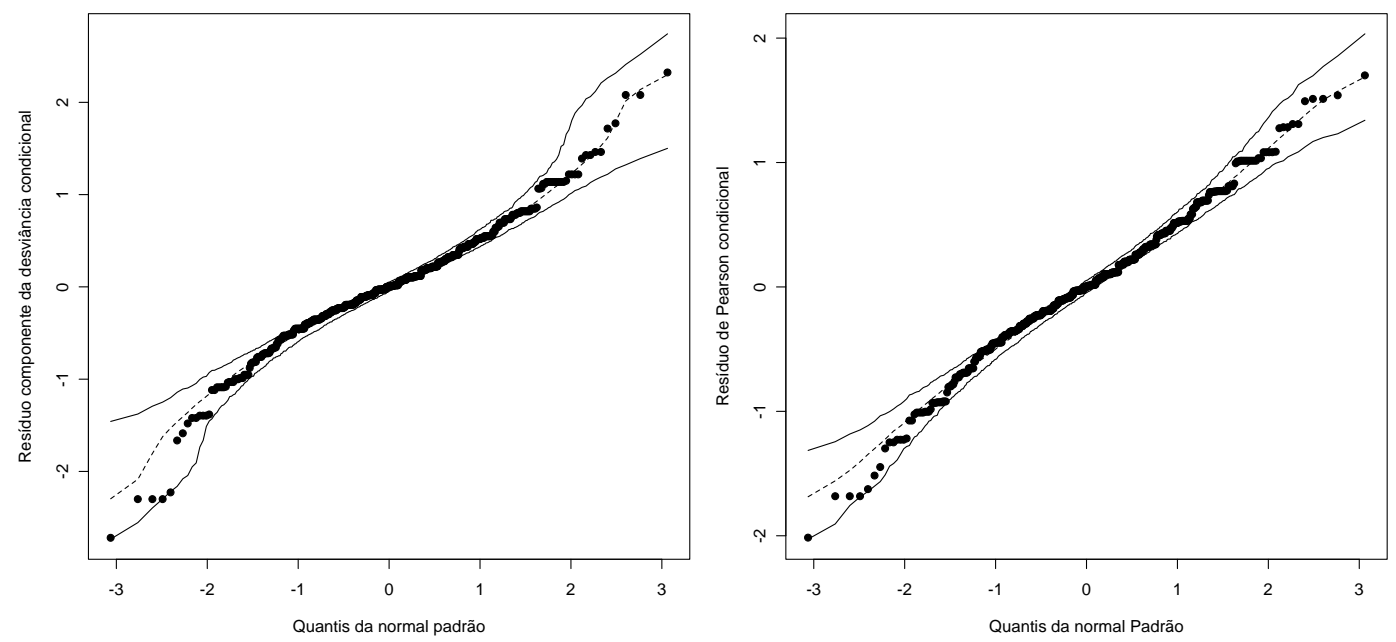

Figura 4.3: Envelopes para o ajuste de QG3 com 99 \% de confiança 


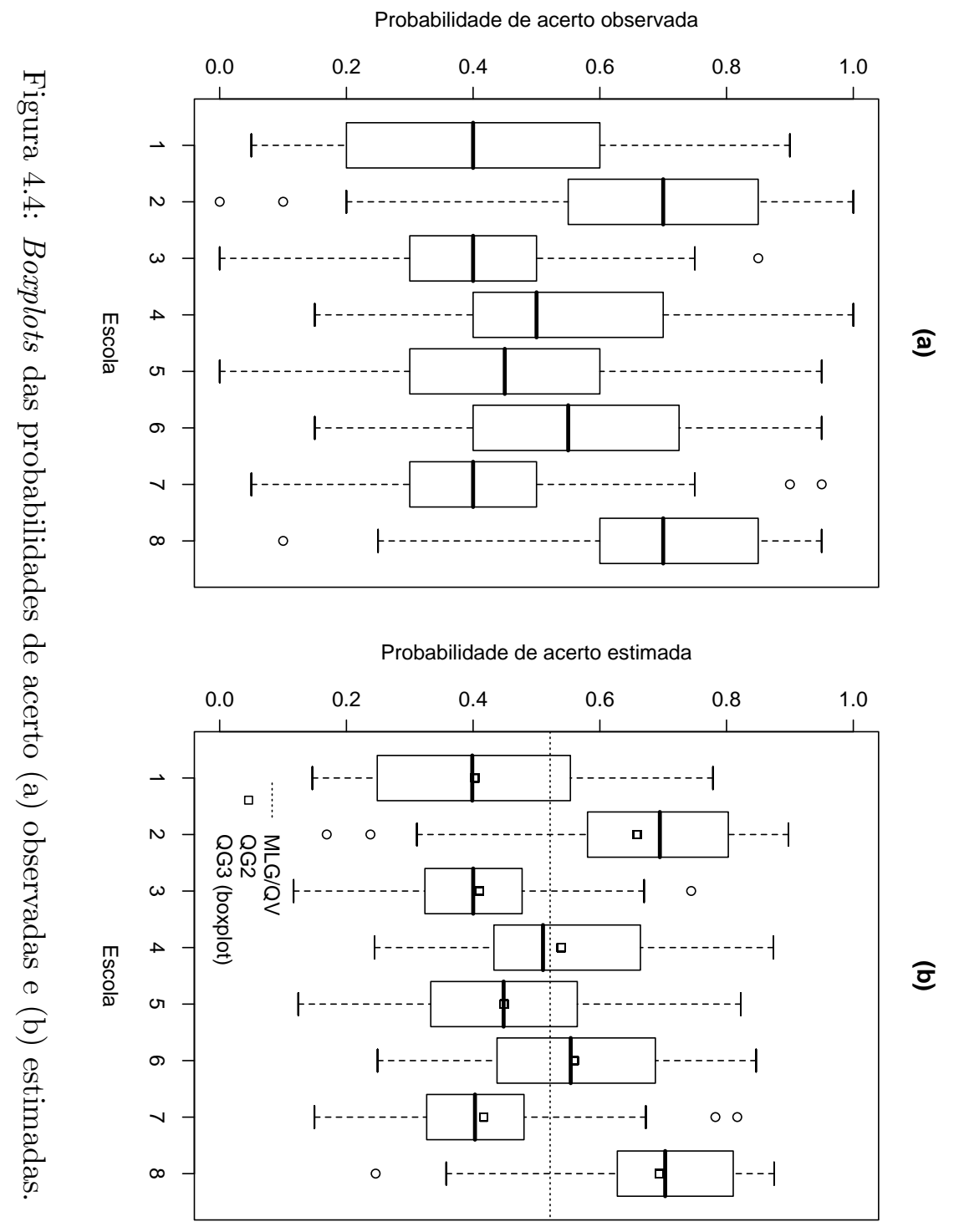




\section{Capítulo 5}

\section{Conclusões}

Este trabalho avaliou a introdução de um efeito aleatório no preditor linear do primeiro nível de um modelo linear generalizado hierárquico (MLGH) binomial-normal em dois níveis, de modo a contemplar a dependência entre respostas binárias de uma mesma unidade experimental do nível 1, uma das possíveis fontes de superdispersão. Uma aplicação para esta situação é a avaliação do desempenho de alunos em uma prova por meio do número de acertos (escore), em que alunos e escolas representam as unidades do nível 1 e do nível 2, respectivamente.

Foram comparados 3 diferentes modelos com resposta binomial: o primeiro (Modelo 1) ajustado por meio da teoria usual de modelos lineares generalizados (MLGs), tanto por máxima verossimilhança (MV) quanto por quase-verossimilhança (QV); o segundo (Modelo 2) ajustado por meio da teoria de MLGHs em 2 níveis, fazendo uso de dois ajustes sob MV, utilizando a quadratura de Gauss-Hermite adaptativa (QGHA) e, por fim, o terceiro modelo (Modelo 3), em que se propõe adicionar um efeito aleatório ao nível 1 do MLGH binomial-normal e estimá-lo sob MV, também fazendo uso da QGHA.

Segundo os resultados de um estudo de simulação, foram discutidos os seguintes aspectos: 1) viés do estimador do componente de variância do nível 1 e do nível 2 ( $\sigma$ e $\tau_{0}$ respectivamente); 2) viés do estimador de $\gamma_{00}$, que é a constante no preditor linear; 3) erro padrão (EP) de $\left.\hat{\gamma}_{00} ; 4\right)$ taxa de rejeição empírica do teste de hipóteses $\mathrm{H}_{0}: \gamma_{00}=\gamma_{00}{ }^{*}$ vs $\mathrm{H}_{\mathrm{a}}: \gamma_{00} \neq \gamma_{00}{ }^{*}$, em que $\gamma_{00}{ }^{*}$ corresponde ao valor real do parâmetro, previamente fixado. 
Houve grande dificuldade em empregar o método de QGHA para aproximar integrais em efeitos aleatórios de diferentes níveis, visto que o aumento, principalmente, no número de unidades do nível 1 aumentou consideravelmente o tempo computacional para a obtenção das estimativas. Este tempo tornou-se ainda maior com o emprego de métodos de otimização para selecionar pontos de quadratura em regiões que promovessem maior contribuição para a aproximação das integrais requeridas. O mapeamento do tempo e recursos computacionais utilizados podem ser de grande valor para pesquisas futuras.

Diante das dificuldades operacionais para ampliar os valores fixados para os parâmetros no estudo de simulação, as conclusões devem ser avaliadas como um estudo preliminar.

Para $\tau_{0}$ e $\sigma$ nulos, as estimativas de $\tau_{0}$, em média, foram similares entre os três modelos. Na presença do componente de variância $\tau_{0}$, em geral, o Modelo 3 apresentou menor viés para seu estimador do que o Modelo 2, principalmente na presença do componente de variância $\sigma$. Estes resultados indicam que o Modelo 3 é relativamente flexível com relação a reproduzir adequadamente os ajustes de seus submodelos.

Na presença apenas do componente $\sigma$, o Modelo 3 produziu, em média, estimativas de $\tau_{0}$ muito mais próximas de zero do que o Modelo 2. O viés do estimador de $\tau_{0}$ apresentou magnitude de 25 a $33 \%$ em relação ao viés obtido com o Modelo 2.

As taxas de rejeição empíricas de QGR e QG2 ficaram próximas às de QG3 em $\gamma_{00}=0$, enquanto que em $\gamma_{00}=1$ em geral isto não ocorreu quando $\sigma \neq 0$, ou seja, quando $\gamma_{00}$ representa diferentes entidades nos Modelos 2 e 3.

Na presença de um efeito aleatório no nível 2, induzindo dependência entre alunos da mesma escola, o aumento no número de alunos $\left(n_{j}\right)$ não contribuiu tanto quanto o aumento no número de escolas $(J)$ para aumentar a precisão e diminuir o viés na estimação dos parâmetros. Especula-se que, analogamente, o aumento do número de respostas oriundas do mesmo aluno não contribua tanto quanto o aumento do número de alunos e/ou de escolas quando há um efeito aleatório no nível 1, uma vez que este efeito induz dependência entre as respostas de um mesmo indivíduo deste nível. Assim, sugere-se ampliar o estudo de simulação para outros conjuntos de parâmetros e tamanhos de amostra maiores, avaliando também diferentes números de respostas para um mesmo indivíduo. É desejável aumentar o número de réplicas das simulações para cada combinação dos 
parâmetros populacionais e tamanhos de amostra, a fim de se aumentar a precisão das estimativas dos valores esperados, eliminando possíveis flutuações ao acaso presentes nos resultados. Outro ponto importante a ser investigado é a inclusão de covariáveis no modelo, de modo a contemplar qualquer variabilidade originada por fatores determinísticos, quase sempre presente em dados reais. É conveniente desenvolver ferramentas para testar a significância dos componentes de variância, hipóteses nulas $\tau_{0}=0$ e/ou $\sigma=0$, que por serem testes na fronteira do espaço paramétrico inviabilizam a utilização da teoria assintótica convencional uma vez que as condições de regularidades usuais não estão satisfeitas. 



\section{Apêndice A}

\section{Esperanças e covariâncias marginais}

Suponha que

$$
Y_{i j} \mid p_{i j} \sim \operatorname{binomial}\left(K, p_{i j}\right), \text { condicionalmente independentes } \forall i \text { e } \forall j
$$

em que $i=1,2, \ldots, n_{j}$ é a identificação do aluno e $j=1,2, \ldots, J$ é a identificação da escola.

Considere o MLGH a seguir.

Nível 1: $\quad \log \left(\frac{p_{i j}}{1-p_{i j}}\right)=\beta_{0 j}+\beta_{1 j} X_{1 i j}+\beta_{2 j} X_{2 i j}+\cdots+\beta_{Q j} X_{Q i j}+a_{i j}$,

e

$$
\begin{aligned}
& \text { Nível 2: } \quad \begin{aligned}
\beta_{0 j} & =\gamma_{00}+\sum_{s=1}^{S_{0}} \gamma_{0 s} W_{0 s j}+\varepsilon_{0 j}, \\
\beta_{q j} & =\gamma_{q 0}+\sum_{s=1}^{S_{q}} \gamma_{q s} W_{q s j}
\end{aligned}
\end{aligned}
$$

em que $i$ e $j$ correspondem às unidades dos níveis 1 e 2 , respectivamente. Cada variável explicativa $X_{q i j}$ está associada à $i$-ésima unidade do nível 1 pertencente à $j$-ésima unidade do nível 2 , com $q=0,1,2, \ldots, Q$ e $X_{0 i j}=1$. Já $W_{q s j}$ representa uma variável independente associada ao $j$-ésimo grupo, $s=1,2, \ldots, S_{q}$. No nível 1 tem-se os efeitos aleatórios $a_{i j}$, independentes $\forall i$ e $\forall j$, com $\mathrm{E}\left[a_{i j}\right]=0$ e $\operatorname{Var}\left[a_{i j}\right]=\sigma^{2}$. No nível 2 apenas o coeficiente $\beta_{0 j}$ apresenta um componente aleatório, 
dado pelos erros $\varepsilon_{0 j}$, independentes $\forall j$, sendo $\mathrm{E}\left[\varepsilon_{0 j}\right]=0$ e $\operatorname{Var}\left[\varepsilon_{0 j}\right]=\tau_{0}^{2}$. Considere também que $\varepsilon_{0 j}$ e $a_{i j}$ são independentes.

Observa-se que a fusão dos dois níveis do modelo resulta numa combinação linear de efeitos fixos somada aos erros aleatórios $a_{i j}$ e $\varepsilon_{0 j}$. Para simplificar a notação, esta combinação linear será indicada como uma constante $c_{i j}$.

\section{A.1 Cálculo de $\mathrm{E}\left[p_{i j}\right], \operatorname{Var}\left[p_{i j}\right]$ e $\operatorname{Cov}\left[p_{i j}, p_{i^{\prime} j}\right]$}

Sejam $\boldsymbol{b}_{j}$ um vetor de erros aleatórios com dimensão $\left(n_{j}+1\right)$ e $\boldsymbol{G}_{j}\left(\boldsymbol{b}_{j}\right)=\left[g_{i}\left(\boldsymbol{b}_{j}\right)\right]_{i=1}^{n_{j}}$ um vetor de funções diferenciáveis.

Sob o Modelo A.2, $p_{i j}$ pode ser escrita como

$$
p_{i j}=\left(1+e^{-c_{i j}-\boldsymbol{A}_{i j}^{T} \boldsymbol{b}_{j}}\right)^{-1}=g_{i}\left(\boldsymbol{b}_{j}\right)
$$

em que $c_{i j}$ é uma combinação linear de efeitos fixos, $\boldsymbol{b}_{j}=\left[\varepsilon_{0 j}, a_{1 j}, a_{2 j}, \ldots, a_{n_{j} j}\right], \mathrm{E}\left[\boldsymbol{b}_{j}\right]=\mathbf{0}$, $\operatorname{Cov}\left[\boldsymbol{b}_{j}\right]=\boldsymbol{\Sigma}$ e $\boldsymbol{A}_{i j}=\left[1, I_{1 j}, I_{2 j}, \ldots, I_{n_{j} j}\right]^{T}$, com $I_{t j}=1$ se $t=i$ e $I_{t j}=0$ caso contrário.

Ainda sob o Modelo A.2,

$$
\frac{\partial g_{i}\left(\boldsymbol{b}_{j}\right)}{\partial \varepsilon_{0 j}}=e^{-c_{i j}-\boldsymbol{A}_{i j}^{T} \boldsymbol{b}_{j}}\left(1+e^{-c_{i j}-\boldsymbol{A}_{i j}^{T} \boldsymbol{b}_{j}}\right)^{-2}
$$

e

$$
\frac{\partial g_{i}\left(\boldsymbol{b}_{j}\right)}{\partial a_{t j}}=\left\{\begin{array}{cl}
\frac{\partial g_{i}\left(\boldsymbol{b}_{j}\right)}{\partial \varepsilon_{0 j}} & \text { se } t=i, t=1,2, \ldots, n_{j} \\
0 & \text { caso contrário }
\end{array}\right.
$$

de modo que

$$
\boldsymbol{G}_{j}^{\prime}\left(\boldsymbol{b}_{j}\right)=\operatorname{diag}\left[\frac{\partial g_{1}\left(\boldsymbol{b}_{j}\right)}{\partial \varepsilon_{0 j}}, \frac{\partial g_{2}\left(\boldsymbol{b}_{j}\right)}{\partial \varepsilon_{0 j}}, \ldots, \frac{\partial g_{n_{j}}\left(\boldsymbol{b}_{j}\right)}{\partial \varepsilon_{0 j}}\right]\left[\boldsymbol{A}_{1 j}^{T}, \boldsymbol{A}_{2 j}^{T}, \ldots, \boldsymbol{A}_{n_{j} j}^{T}\right]^{T} .
$$

Aproximando o vetor $\boldsymbol{G}_{j}\left(\boldsymbol{b}_{j}\right)$ por meio de uma expansão de Taylor de primeira ordem em 
torno de $\mathrm{E}\left[\boldsymbol{b}_{j}\right]=\mathbf{0}$, tem-se

$$
\boldsymbol{G}_{j}\left(\boldsymbol{b}_{j}\right) \cong \boldsymbol{G}_{j}\left(\mathrm{E}\left[\boldsymbol{b}_{j}\right]\right)+\boldsymbol{G}_{j}^{\prime}\left(\mathrm{E}\left[\boldsymbol{b}_{j}\right]\right)\left(\boldsymbol{b}_{j}-\mathrm{E}\left[\boldsymbol{b}_{j}\right]\right)
$$

de modo que

$$
\mathrm{E}\left[\boldsymbol{G}_{j}\left(\boldsymbol{b}_{j}\right)\right] \cong \boldsymbol{G}_{j}\left(\mathrm{E}\left[\boldsymbol{b}_{j}\right]\right)+\boldsymbol{G}_{j}^{\prime}\left(\mathrm{E}\left[\boldsymbol{b}_{j}\right]\right)\left(\mathrm{E}\left[\boldsymbol{b}_{j}\right]-\mathrm{E}\left[\boldsymbol{b}_{j}\right]\right)=\boldsymbol{G}_{j}\left(\mathrm{E}\left[\boldsymbol{b}_{j}\right]\right)=\boldsymbol{G}_{j}(\mathbf{0})
$$

e

$$
\operatorname{Cov}\left[\boldsymbol{G}_{j}\left(\boldsymbol{b}_{j}\right)\right] \cong \boldsymbol{G}_{j}^{\prime}\left(\mathrm{E}\left[\boldsymbol{b}_{j}\right]\right) \operatorname{Var}\left[\boldsymbol{b}_{j}\right] \boldsymbol{G}_{j}^{\prime}\left(\mathrm{E}\left[\boldsymbol{b}_{j}\right]\right)^{T}=\boldsymbol{G}_{j}^{\prime}(\mathbf{0}) \boldsymbol{\Sigma} \boldsymbol{G}_{j}^{\prime}(\mathbf{0})^{T}
$$

Considere $i=1,2$ e $\boldsymbol{b}_{j}=\left[\varepsilon_{0 j}, a_{1 j}, a_{2 j}\right]^{T}$, com $\mathrm{E}\left[\boldsymbol{b}_{j}\right]=\mathbf{0}$ e $\boldsymbol{\Sigma}=\operatorname{diag}\left[\tau_{0}^{2}, \sigma^{2}, \sigma^{2}\right]$. Nesse caso $\boldsymbol{G}_{j}\left(\boldsymbol{b}_{j}\right)=\left[g_{1}\left(\boldsymbol{b}_{j}\right), g_{2}\left(\boldsymbol{b}_{j}\right)\right]^{T}$ e $\boldsymbol{A}_{i j}=\left[1, I_{1 j}, I_{2 j}\right]^{T} \mathrm{e}$

$$
\frac{\partial g_{i}\left(\boldsymbol{b}_{j}\right)}{\partial \varepsilon_{0 j}}=\frac{\partial g_{i}\left(\boldsymbol{b}_{j}\right)}{\partial a_{i j}}=e^{-c_{i j}-\varepsilon_{0 j}-a_{i j}}\left[1+e^{-c_{i j}-\varepsilon_{0 j}-a_{i j}}\right]^{-2}
$$

de modo que a equação (A.9) fica expressa por

$$
\begin{aligned}
\operatorname{Cov}\left[\boldsymbol{G}_{j}\left(\boldsymbol{b}_{j}\right)\right] & \cong\left[\begin{array}{cc}
\tau_{0}^{2}\left(\frac{\partial g_{1}(\mathbf{0})}{\partial \varepsilon_{0 j}}\right)^{2}+\sigma^{2}\left(\frac{\partial g_{1}(\mathbf{0})}{\partial a_{1 j}}\right)^{2} & \tau_{0}^{2} \frac{\partial g_{1}(\mathbf{0})}{\partial \varepsilon_{0 j}} \frac{\partial g_{2}(\mathbf{0})}{\partial \varepsilon_{0 j}} \\
\tau_{0}^{2} \frac{\partial g_{1}(\mathbf{0})}{\partial \varepsilon_{0 j}} \frac{\partial g_{2}(\mathbf{0})}{\partial \varepsilon_{0 j}} & \tau_{0}^{2}\left(\frac{\partial g_{2}(\mathbf{0})}{\partial \varepsilon_{0 j}}\right)^{2}+\sigma^{2}\left(\frac{\partial g_{2}(\mathbf{0})}{\partial a_{2 j}}\right)^{2}
\end{array}\right] \\
& \cong\left[\begin{array}{cc}
\left(\tau_{0}^{2}+\sigma^{2}\right)\left(\frac{\partial g_{1}(\mathbf{0})}{\partial \varepsilon_{0 j}}\right)^{2} & \tau_{0}^{2} \frac{\partial g_{1}(\mathbf{0})}{\partial \varepsilon_{0 j}} \frac{\partial g_{2}(\mathbf{0})}{\partial \varepsilon_{0 j}} \\
\tau_{0}^{2} \frac{\partial g_{1}(\mathbf{0})}{\partial \varepsilon_{0 j}} \frac{\partial g_{2}(\mathbf{0})}{\partial \varepsilon_{0 j}} & \left(\tau_{0}^{2}+\sigma^{2}\right)\left(\frac{\partial g_{2}(\mathbf{0})}{\partial \varepsilon_{0 j}}\right)^{2}
\end{array}\right] .
\end{aligned}
$$

Supondo que o vetor $\boldsymbol{b}_{j}$ tenha uma pequena variação em torno de $\mathrm{E}\left[\boldsymbol{b}_{j}\right]=\mathbf{0}$, conclui-se então que

$$
\begin{aligned}
\mathrm{E}\left[p_{i j}\right] & \cong\left(1+e^{-c_{i j}}\right)^{-1} \\
\operatorname{Var}\left[p_{i j}\right] & \cong\left(\tau_{0}^{2}+\sigma^{2}\right)\left[e^{-c_{i j}}\left(1+e^{-c_{i j}}\right)^{-2}\right]^{2} \\
\operatorname{Cov}\left[p_{i j}, p_{i^{\prime} j}\right] & \cong \tau_{0}^{2} e^{-c_{1 j}-c_{2 j}}\left[\left(1+e^{-c_{1 j}}\right)\left(1+e^{-c_{2 j}}\right)\right]^{-2}
\end{aligned}
$$

e

$$
\operatorname{Corr}\left[p_{i j}, p_{i^{\prime} j}\right] \cong \tau_{0}^{2}\left(\tau_{0}^{2}+\sigma^{2}\right)^{-1}
$$




\section{A.2 Cálculo de $\mathrm{E}\left[Y_{i j}\right], \operatorname{Var}\left[Y_{i j}\right]$ e $\operatorname{Cov}\left[Y_{i j}, Y_{i^{\prime} j}\right]$}

$$
\begin{aligned}
& \mathrm{E}\left[Y_{i j}\right]=\mathrm{E}\left\{\mathrm{E}\left[Y_{i j} \mid p_{i j}\right]\right\}=K \mathrm{E}\left[p_{i j}\right] \cong K\left(1+e^{-c_{i j}}\right)^{-1} \\
& \operatorname{Var}\left[Y_{i j}\right]=\mathrm{E}\left\{\operatorname{Var}\left[Y_{i j} \mid p_{i j}\right]\right\}+\operatorname{Var}\left\{\mathrm{E}\left[Y_{i j} \mid p_{i j}\right]\right\} \\
& =\mathrm{E}\left[\phi K p_{i j}\left(1-p_{i j}\right)\right]+\operatorname{Var}\left[K p_{i j}\right] \\
& =\phi K\left(\mathrm{E}\left[p_{i j}\right]-\mathrm{E}\left[p_{i j}^{2}\right]\right)+K^{2} \operatorname{Var}\left[p_{i j}\right] \\
& =\phi K\left(\mathrm{E}\left[p_{i j}\right]-\mathrm{E}^{2}\left[p_{i j}\right]-\operatorname{Var}\left[p_{i j}\right]\right)+K^{2} \operatorname{Var}\left[p_{i j}\right] \\
& =\phi K \mathrm{E}\left[p_{i j}\right]\left(1-\mathrm{E}\left[p_{i j}\right]\right)+K(K-\phi) \operatorname{Var}\left[p_{i j}\right] \\
& \cong \phi K e^{-c_{i j}}\left(1+e^{-c_{i j}}\right)^{-2}+K(K-\phi)\left(\tau_{0}^{2}+\sigma^{2}\right)\left[e^{-c_{i j}}\left(1+e^{-c_{i j}}\right)^{-2}\right]^{2} \\
& \cong K e^{-c_{i j}\left(1+e^{-c_{i j}}\right)^{-2}\left[\phi+\left(\tau_{0}^{2}+\sigma^{2}\right)(K-\phi) e^{-c_{i j}}\left(1+e^{-c_{i j}}\right)^{-2}\right]} \text {, }
\end{aligned}
$$

$$
\begin{aligned}
\operatorname{Cov}\left[Y_{i j}, Y_{i^{\prime} j}\right] & =\mathrm{E}\left[Y_{i j} Y_{i^{\prime} j}\right]-\mathrm{E}\left[Y_{i j}\right] \mathrm{E}\left[Y_{i^{\prime} j}\right] \\
& =\mathrm{E}\left\{\mathrm{E}\left[Y_{i j} Y_{i^{\prime} j} \mid p_{i j}, p_{i^{\prime} j}\right]\right\}-\mathrm{E}\left\{\mathrm{E}\left[Y_{i j} \mid p_{i j}\right]\right\} \mathrm{E}\left\{\mathrm{E}\left[Y_{i^{\prime} j} \mid p_{i^{\prime} j}\right]\right\} \\
& =\mathrm{E}\left\{\mathrm{E}\left[Y_{i j} \mid p_{i j}\right] \mathrm{E}\left[Y_{i^{\prime} j} \mid p_{i^{\prime} j}\right]\right\}-K^{2} \mathrm{E}\left[p_{i j}\right] \mathrm{E}\left[p_{i^{\prime} j}\right] \\
& =K^{2} \mathrm{E}\left[p_{i j} p_{i^{\prime} j}\right]-K^{2} \mathrm{E}\left[p_{i j}\right] \mathrm{E}\left[p_{i^{\prime} j}\right] \\
& =K^{2} \operatorname{Cov}\left[p_{i j}, p_{i^{\prime} j}\right] \\
& \cong K^{2} \tau_{0}^{2} e^{-c_{i j}-c_{i^{\prime} j}}\left[\left(1+e^{-c_{i j}}\right)\left(1+e^{-c_{i^{\prime} j}}\right)\right]^{-2}
\end{aligned}
$$

e

$$
\begin{aligned}
\operatorname{Corr}\left[Y_{i j}, Y_{i^{\prime} j}\right] & =\frac{\operatorname{Cov}\left[Y_{i j}, Y_{i^{\prime} j}\right]}{\left\{\operatorname{Var}\left[Y_{i j}\right] \operatorname{Var}\left[Y_{i^{\prime} j}\right]\right\}^{1 / 2}} \\
& \cong \prod_{t \in\left(i, i^{\prime}\right)} \frac{K^{2} \tau_{0}^{2} e^{-c_{t j}}\left(1+e^{-c_{t j}}\right)^{-2}}{\left\{K e^{-c_{t j}}\left(1+e^{-c_{t j}}\right)^{-2}\left[\phi+(K-\phi)\left(\tau_{0}^{2}+\sigma^{2}\right) e^{-c_{t j}}\left(1+e^{-c_{t j}}\right)^{-2}\right]\right\}^{1 / 2}} \\
& \cong K \tau_{0}^{2} \prod_{t \in\left(i, i^{\prime}\right)}\left[\frac{e^{-c_{t j}}\left(1+e^{-c_{t j}}\right)^{-2}}{\phi+(K-\phi)\left(\tau_{0}^{2}+\sigma^{2}\right) e^{-c_{t j}}\left(1+e^{-c_{t j}}\right)^{-2}}\right]^{1 / 2} \\
& \cong K \tau_{0}^{2} \prod_{t \in\left(i, i^{\prime}\right)}\left\{\phi\left[e^{-c_{t j}}\left(1+e^{-c_{t j}}\right)^{-2}\right]^{-1}+\left(\tau_{0}^{2}+\sigma^{2}\right)(K-\phi)\right\}^{-1 / 2}
\end{aligned}
$$


Apêndice B

\section{Tabelas}


Tabela B.1: Média das estimativas de $\tau_{0}$ sob QGR, QG2 e QG3 e de $\sigma$ sob QG3, quando $\gamma_{00}=0$.

\begin{tabular}{|c|c|c|c|c|c|c|c|c|c|}
\hline \multicolumn{4}{|c|}{ Valores fixados } & \multicolumn{3}{|c|}{$\begin{array}{c}\text { Média das estimativas } \\
\text { de } \tau_{0}\end{array}$} & \multicolumn{2}{|c|}{$\begin{array}{l}\text { \% relativa } \\
\text { a QG3 }\end{array}$} & \multirow{2}{*}{$\begin{array}{l}\text { Média das } \\
\text { estimativas } \\
\quad \text { de } \sigma\end{array}$} \\
\hline$\tau_{0}$ & $\sigma$ & $J$ & $n_{j}$ & QGR & QG2 & QG3 & QGR & QG2 & \\
\hline 0 & 0 & 20 & 20 & 0.0178 & 0.0181 & 0.0174 & 102 & 104 & 0.0433 \\
\hline 0 & 0 & 20 & 50 & 0.0108 & 0.0111 & 0.0111 & 98 & 100 & 0.0357 \\
\hline 0 & 0 & 50 & 20 & 0.0174 & 0.0178 & 0.0170 & 103 & 105 & 0.0351 \\
\hline 0 & 0 & 50 & 50 & 0.0100 & 0.0105 & 0.0103 & 98 & 102 & 0.0296 \\
\hline 0.5 & 0 & 20 & 20 & $\overline{0.4794}$ & 0.4794 & 0.5047 & 95 & 95 & 0.0535 \\
\hline 0.5 & 0 & 20 & 50 & 0.4794 & 0.4794 & 0.6358 & 75 & 75 & 0.0478 \\
\hline 0.5 & 0 & 50 & 20 & 0.4899 & 0.4899 & 0.5252 & 93 & 93 & 0.0451 \\
\hline 0.5 & 0 & 50 & 50 & 0.4925 & 0.4925 & 0.6701 & 73 & 73 & 0.0413 \\
\hline 1 & 0 & 20 & 20 & 0.9571 & 0.9571 & 0.9600 & 100 & 100 & 0.0564 \\
\hline 1 & 0 & 20 & 50 & 0.9656 & 0.9656 & 0.9737 & 99 & 99 & 0.0436 \\
\hline 1 & 0 & 50 & 20 & 0.9884 & 0.9884 & 0.9895 & 100 & 100 & 0.0413 \\
\hline 1 & 0 & 50 & 50 & 0.9859 & 0.9858 & 0.9897 & 100 & 100 & 0.0348 \\
\hline 0 & 0.5 & 20 & 20 & $\overline{0.0934}$ & 0.0934 & 0.0294 & 318 & 318 & 0.4953 \\
\hline 0 & 0.5 & 20 & 50 & 0.0580 & 0.0580 & 0.0192 & 302 & 302 & 0.4973 \\
\hline 0 & 0.5 & 50 & 20 & 0.0985 & 0.0985 & 0.0242 & 408 & 408 & 0.4979 \\
\hline 0 & 0.5 & 50 & 50 & 0.0626 & 0.0626 & 0.0161 & 389 & 389 & 0.4989 \\
\hline 0 & 1 & 20 & 20 & 0.1716 & 0.1716 & 0.0460 & 373 & 373 & 0.9949 \\
\hline 0 & 1 & 20 & 50 & 0.1094 & 0.1094 & 0.0307 & 357 & 357 & 0.9980 \\
\hline 0 & 1 & 50 & 20 & 0.1794 & 0.1794 & 0.0417 & 431 & 431 & 0.9956 \\
\hline 0 & 1 & 50 & 50 & 0.1126 & 0.1126 & 0.0259 & 436 & 436 & 0.9983 \\
\hline 0.5 & 0.5 & 20 & 20 & 0.4636 & 0.4636 & 0.4764 & 97 & 97 & 0.4992 \\
\hline 0.5 & 0.5 & 20 & 50 & 0.4587 & 0.4587 & 0.5149 & 89 & 89 & 0.5001 \\
\hline 0.5 & 0.5 & 50 & 20 & 0.4768 & 0.4768 & 0.4914 & 97 & 97 & 0.4995 \\
\hline 0.5 & 0.5 & 50 & 50 & 0.4679 & 0.4679 & 0.5374 & 87 & 87 & 0.5010 \\
\hline 0.5 & 1 & 20 & 20 & $\overline{0.4345}$ & 0.4345 & 0.4677 & 93 & 93 & 1.0012 \\
\hline 0.5 & 1 & 20 & 50 & 0.4148 & 0.4149 & 0.4778 & 87 & 87 & 0.9989 \\
\hline 0.5 & 1 & 50 & 20 & 0.4498 & 0.4498 & 0.4889 & 92 & 92 & 0.9995 \\
\hline 0.5 & 1 & 50 & 50 & 0.4252 & 0.4252 & 0.4917 & 86 & 86 & 1.0006 \\
\hline 1 & 0.5 & 20 & 20 & $\overline{0.9265}$ & 0.9265 & 0.9643 & 96 & 96 & 0.5007 \\
\hline 1 & 0.5 & 20 & 50 & 0.9124 & 0.9124 & 0.9553 & 96 & 96 & 0.5001 \\
\hline 1 & 0.5 & 50 & 20 & 0.9480 & 0.9480 & 0.9874 & 96 & 96 & 0.4987 \\
\hline 1 & 0.5 & 50 & 50 & 0.9419 & 0.9419 & 0.9862 & 96 & 96 & 0.5000 \\
\hline 1 & 1 & 20 & 20 & 0.8425 & 0.8425 & 0.9682 & 87 & 87 & 0.9997 \\
\hline 1 & 1 & 20 & 50 & 0.8166 & 0.8166 & 0.9574 & 85 & 85 & 0.9987 \\
\hline 1 & 1 & 50 & 20 & 0.8535 & 0.8535 & 0.9818 & 87 & 87 & 1.0008 \\
\hline 1 & 1 & 50 & 50 & 0.8394 & 0.8395 & 0.9849 & 85 & 85 & 0.9996 \\
\hline
\end{tabular}


Tabela B.2: Média das estimativas de $\tau_{0}$ sob QGR, QG2 e QG3 e de $\sigma$ sob QG3, quando $\gamma_{00}=1$.

\begin{tabular}{|c|c|c|c|c|c|c|c|c|c|}
\hline \multicolumn{4}{|c|}{ Valores fixados } & \multicolumn{3}{|c|}{$\begin{array}{c}\text { Média das estimativas } \\
\text { de } \tau_{0}\end{array}$} & \multicolumn{2}{|c|}{$\begin{array}{l}\% \text { relativa } \\
\text { a QG3 }\end{array}$} & \multirow{2}{*}{$\begin{array}{c}\text { Média das } \\
\text { estimativas } \\
\text { de } \sigma\end{array}$} \\
\hline$\tau_{0}$ & $\sigma$ & $J$ & $n_{j}$ & QGR & QG2 & QG3 & QGR & QG2 & \\
\hline 0 & 0 & 20 & 20 & 0.0210 & 0.0212 & 0.0197 & 107 & 108 & 0.0489 \\
\hline 0 & 0 & 20 & 50 & 0.0123 & 0.0126 & 0.0118 & 105 & 108 & 0.0406 \\
\hline 0 & 0 & 50 & 20 & 0.0184 & 0.0189 & 0.0175 & 106 & 108 & 0.0404 \\
\hline 0 & 0 & 50 & 50 & 0.0118 & 0.0123 & 0.0113 & 105 & 109 & 0.0321 \\
\hline 0.5 & 0 & 20 & 20 & 0.4802 & 0.4802 & 0.4968 & 97 & 97 & 0.0590 \\
\hline 0.5 & 0 & 20 & 50 & 0.4834 & 0.4834 & 0.6109 & 79 & 79 & 0.0511 \\
\hline 0.5 & 0 & 50 & 20 & 0.4941 & 0.4941 & 0.5202 & 95 & 95 & 0.0480 \\
\hline 0.5 & 0 & 50 & 50 & 0.4919 & 0.4919 & 0.6494 & 76 & 76 & 0.0424 \\
\hline 1 & 0 & 20 & 20 & 0.9608 & 0.9608 & 0.9613 & 100 & 100 & 0.0641 \\
\hline 1 & 0 & 20 & 50 & 0.9604 & 0.9604 & 0.9671 & 99 & 99 & 0.0484 \\
\hline 1 & 0 & 50 & 20 & 0.9848 & 0.9848 & 0.9859 & 100 & 100 & 0.0482 \\
\hline 1 & 0 & 50 & 50 & 0.9826 & 0.9826 & 0.9874 & 100 & 100 & 0.0370 \\
\hline 0 & 0.5 & 20 & 20 & 0.0925 & 0.0926 & 0.0306 & 302 & 302 & 0.4961 \\
\hline 0 & 0.5 & 20 & 50 & 0.0585 & 0.0586 & 0.0195 & 300 & 300 & 0.4978 \\
\hline 0 & 0.5 & 50 & 20 & 0.1000 & 0.1001 & 0.0258 & 388 & 388 & 0.4966 \\
\hline 0 & 0.5 & 50 & 50 & 0.0634 & 0.0634 & 0.0164 & 387 & 387 & 0.4984 \\
\hline 0 & 1 & 20 & 20 & 0.1768 & 0.1768 & 0.0432 & 410 & 410 & 0.9961 \\
\hline 0 & 1 & 20 & 50 & 0.1125 & 0.1125 & 0.0295 & 381 & 381 & 0.9986 \\
\hline 0 & 1 & 50 & 20 & 0.1858 & 0.1858 & 0.0425 & 437 & 437 & 0.9971 \\
\hline 0 & 1 & 50 & 50 & 0.1169 & 0.1170 & 0.0271 & 431 & 431 & 0.9984 \\
\hline 0.5 & 0.5 & 20 & 20 & 0.4722 & 0.4722 & 0.4813 & 98 & 98 & 0.4994 \\
\hline 0.5 & 0.5 & 20 & 50 & 0.4679 & 0.4679 & 0.5154 & 91 & 91 & 0.5003 \\
\hline 0.5 & 0.5 & 50 & 20 & 0.4840 & 0.4840 & 0.4949 & 98 & 98 & 0.4997 \\
\hline 0.5 & 0.5 & 50 & 50 & 0.4751 & 0.4751 & 0.5359 & 89 & 89 & 0.5020 \\
\hline 0.5 & 1 & 20 & 20 & 0.4492 & 0.4492 & 0.4753 & 95 & 95 & 0.9993 \\
\hline 0.5 & 1 & 20 & 50 & 0.4196 & 0.4196 & 0.4744 & 88 & 88 & 0.9996 \\
\hline 0.5 & 1 & 50 & 20 & 0.4598 & 0.4598 & 0.4896 & 94 & 94 & 0.9998 \\
\hline 0.5 & 1 & 50 & 50 & 0.4343 & 0.4343 & 0.4930 & 88 & 88 & 0.9998 \\
\hline 1 & 0.5 & 20 & 20 & 0.9258 & 0.9258 & 0.9568 & 97 & 97 & 0.5000 \\
\hline 1 & 0.5 & 20 & 50 & 0.9311 & 0.9311 & 0.9663 & 96 & 96 & 0.4995 \\
\hline 1 & 0.5 & 50 & 20 & 0.9515 & 0.9515 & 0.9856 & 97 & 97 & 0.4993 \\
\hline 1 & 0.5 & 50 & 50 & 0.9459 & 0.9460 & 0.9858 & 96 & 96 & 0.5002 \\
\hline 1 & 1 & 20 & 20 & 0.8481 & 0.8481 & 0.9597 & 88 & 88 & 0.9996 \\
\hline 1 & 1 & 20 & 50 & 0.8312 & 0.8312 & 0.9582 & 87 & 87 & 0.9992 \\
\hline 1 & 1 & 50 & 20 & 0.8688 & 0.8688 & 0.9846 & 88 & 88 & 0.9996 \\
\hline 1 & 1 & 50 & 50 & 0.8478 & 0.8478 & 0.9804 & 86 & 86 & 0.9993 \\
\hline
\end{tabular}


Tabela B.3: Viés estimado de $\hat{\gamma}_{00}$ sob cada ajuste, quando $\gamma_{00}=0$.

\begin{tabular}{|c|c|c|c|c|c|c|c|c|c|c|}
\hline \multicolumn{4}{|c|}{ Valores fixados } & \multicolumn{4}{|c|}{ Viés } & \multicolumn{3}{|c|}{$\%$ relativa a $\mathrm{QG} 3^{* *}$} \\
\hline$\tau_{0}$ & $\sigma$ & $J$ & $n_{j}$ & MLG* & QGR & QG2 & QG3 & MLG* & QGR & QG2 \\
\hline 0 & 0 & 20 & 20 & -0.00056 & -0.00056 & -0.00056 & -0.00057 & $\begin{array}{r}99.9 \\
\end{array}$ & 99.7 & 99.6 \\
\hline 0 & 0 & 20 & 50 & -0.00014 & -0.00014 & -0.00013 & -0.00014 & 98.9 & 98.9 & 94.6 \\
\hline 0 & 0 & 50 & 20 & -0.00001 & -0.00001 & -0.00001 & -0.00001 & 105.4 & 107.1 & 158.1 \\
\hline 0 & 0 & 50 & 50 & -0.00025 & -0.00025 & -0.00025 & -0.00025 & 99.9 & 100.1 & 100.0 \\
\hline 0.5 & 0 & 20 & 20 & -0.00300 & -0.00315 & -0.00314 & -0.00269 & 111.6 & 117.1 & 117.0 \\
\hline 0.5 & 0 & 20 & 50 & 0.00174 & 0.00166 & 0.00166 & 0.00154 & 112.9 & 107.7 & 107.4 \\
\hline 0.5 & 0 & 50 & 20 & 0.00189 & 0.00214 & 0.00215 & 0.00250 & 75.8 & 85.6 & 85.9 \\
\hline 0.5 & 0 & 50 & 50 & 0.00079 & 0.00081 & 0.00079 & 0.00051 & 153.0 & 158.6 & 154.8 \\
\hline 1 & 0 & 20 & 20 & 0.00458 & 0.00690 & 0.00692 & 0.00702 & 65.3 & 98.3 & 98.5 \\
\hline 1 & 0 & 20 & 50 & -0.00553 & -0.00696 & -0.00697 & -0.00671 & 82.4 & 103.8 & 103.9 \\
\hline 1 & 0 & 50 & 20 & 0.00038 & 0.00075 & 0.00074 & 0.00075 & 50.6 & 98.9 & 98.4 \\
\hline 1 & 0 & 50 & 50 & 0.00180 & 0.00199 & 0.00192 & 0.00194 & 92.5 & 102.5 & 98.6 \\
\hline 0 & 0.5 & 20 & 20 & -0.00101 & -0.00101 & -0.00102 & -0.00106 & 95.4 & 95.6 & 95.9 \\
\hline 0 & 0.5 & 20 & 50 & 0.00015 & 0.00015 & 0.00015 & 0.00017 & 89.8 & 89.8 & 91.3 \\
\hline 0 & 0.5 & 50 & 20 & 0.00017 & 0.00017 & 0.00017 & 0.00018 & 96.9 & 96.2 & 96.1 \\
\hline 0 & 0.5 & 50 & 50 & 0.00037 & 0.00037 & 0.00037 & 0.00039 & 93.6 & 93.8 & 94.9 \\
\hline 0 & 1 & 20 & 20 & 0.00233 & 0.00234 & 0.00235 & 0.00281 & 82.9 & 83.3 & 83.5 \\
\hline 0 & 1 & 20 & 50 & 0.00055 & 0.00056 & 0.00056 & 0.00081 & 68.5 & 68.7 & 69.2 \\
\hline 0 & 1 & 50 & 20 & 0.00047 & 0.00047 & 0.00047 & 0.00044 & 107.4 & 107.9 & 107.1 \\
\hline 0 & 1 & 50 & 50 & 0.00038 & 0.00038 & 0.00039 & 0.00041 & 93.3 & 93.7 & 94.2 \\
\hline 0.5 & 0.5 & 20 & 20 & -0.00144 & -0.00130 & -0.00130 & -0.00127 & 113.5 & 102.3 & 102.3 \\
\hline 0.5 & 0.5 & 20 & 50 & -0.00238 & -0.00242 & -0.00243 & -0.00242 & 98.4 & 100.0 & 100.5 \\
\hline 0.5 & 0.5 & 50 & 20 & 0.00273 & 0.00289 & 0.00289 & 0.00300 & 91.1 & 96.4 & 96.5 \\
\hline 0.5 & 0.5 & 50 & 50 & -0.00030 & -0.00033 & -0.00035 & -0.00037 & 79.9 & 87.7 & 93.2 \\
\hline 0.5 & 1 & 20 & 20 & 0.00003 & 0.00018 & 0.00018 & 0.00023 & 13.8 & 79.0 & 77.1 \\
\hline 0.5 & 1 & 20 & 50 & -0.00649 & -0.00694 & -0.00691 & -0.00835 & 77.8 & 83.2 & 82.8 \\
\hline 0.5 & 1 & 50 & 20 & -0.00021 & -0.00025 & -0.00026 & -0.00031 & 68.4 & 80.7 & 83.8 \\
\hline 0.5 & 1 & 50 & 50 & 0.00086 & 0.00094 & 0.00092 & 0.00119 & 71.9 & 78.7 & 77.1 \\
\hline 1 & 0.5 & 20 & 20 & -0.00071 & -0.00118 & -0.00119 & -0.00102 & 69.0 & 115.9 & 116.6 \\
\hline 1 & 0.5 & 20 & 50 & 0.00231 & 0.00351 & 0.00353 & 0.00413 & 55.9 & 85.1 & 85.5 \\
\hline 1 & 0.5 & 50 & 20 & 0.00291 & 0.00304 & 0.00303 & 0.00325 & 89.6 & 93.6 & 93.3 \\
\hline 1 & 0.5 & 50 & 50 & 0.00294 & 0.00353 & 0.00348 & 0.00378 & 77.7 & 93.3 & 92.1 \\
\hline 1 & 1 & 20 & 20 & 0.00299 & 0.00308 & 0.00307 & 0.00400 & 74.9 & 76.9 & 76.8 \\
\hline 1 & 1 & 20 & 50 & 0.00717 & 0.00790 & 0.00792 & 0.00959 & 74.8 & 82.4 & 82.6 \\
\hline 1 & 1 & 50 & 20 & 0.00069 & 0.00061 & 0.00059 & 0.00060 & 115.2 & 101.9 & 98.3 \\
\hline 1 & 1 & 50 & 50 & 0.00476 & 0.00582 & 0.00581 & 0.00695 & 68.5 & 83.8 & 83.6 \\
\hline
\end{tabular}


Tabela B.4: Viés estimado de $\hat{\gamma}_{00}$ sob cada ajuste, quando $\gamma_{00}=1$.

\begin{tabular}{|c|c|c|c|c|c|c|c|c|c|c|}
\hline \multicolumn{4}{|c|}{ Valores fixados } & \multicolumn{4}{|c|}{ Viés } & \multicolumn{3}{|c|}{$\%$ relativa a $\mathrm{QG} 3^{* *}$} \\
\hline$\tau_{0}$ & $\sigma$ & $J$ & $n_{j}$ & MLG* & QGR & QG2 & QG3 & MLG* & QGR & QG2 \\
\hline 0 & 0 & 20 & 20 & 0.00062 & 0.00092 & 0.00092 & 0.00237 & 26.2 & 39.0 & 38.9 \\
\hline 0 & 0 & 20 & 50 & 0.00006 & 0.00017 & 0.00018 & 0.00112 & 5.5 & 15.6 & 15.8 \\
\hline 0 & 0 & 50 & 20 & 0.00004 & 0.00026 & 0.00026 & 0.00120 & 3.4 & 21.6 & 21.6 \\
\hline 0 & 0 & 50 & 50 & 0.00038 & 0.00046 & 0.00047 & 0.00104 & 36.1 & 44.6 & 45.4 \\
\hline 0.5 & 0 & 20 & 20 & $\overline{-0.05071}$ & -0.00067 & -0.00067 & -0.00532 & 953.4 & 12.6 & 12.6 \\
\hline 0.5 & 0 & 20 & 50 & -0.04986 & 0.00083 & 0.00084 & -0.03015 & 165.4 & -2.8 & -2.8 \\
\hline 0.5 & 0 & 50 & 20 & -0.05520 & -0.00337 & -0.00339 & -0.01321 & 417.9 & 25.5 & 25.7 \\
\hline 0.5 & 0 & 50 & 50 & -0.04986 & 0.00170 & 0.00167 & -0.03893 & 128.1 & -4.4 & -4.3 \\
\hline 1 & 0 & 20 & 20 & -0.17047 & -0.01145 & -0.01142 & -0.01106 & 1541.4 & 103.5 & 103.2 \\
\hline 1 & 0 & 20 & 50 & -0.15814 & 0.00220 & 0.00218 & -0.00083 & 18974.8 & -263.8 & -262.1 \\
\hline 1 & 0 & 50 & 20 & -0.16931 & -0.00499 & -0.00500 & -0.00411 & 4121.0 & 121.6 & 121.8 \\
\hline 1 & 0 & 50 & 50 & -0.17439 & -0.01117 & -0.01114 & -0.01142 & 1526.9 & 97.8 & 97.5 \\
\hline 0 & 0.5 & 20 & 20 & -0.05437 & -0.05212 & -0.05212 & -0.00179 & 3040.6 & 2915.0 & 2915.0 \\
\hline 0 & 0.5 & 20 & 50 & -0.05181 & -0.05091 & -0.05092 & 0.00077 & -6771.0 & -6653.9 & -6654.3 \\
\hline 0 & 0.5 & 50 & 20 & -0.05315 & -0.05082 & -0.05082 & -0.00073 & 7276.7 & 6958.4 & 6957.9 \\
\hline 0 & 0.5 & 50 & 50 & -0.05297 & -0.05204 & -0.05204 & -0.00047 & 11256.0 & 11057.2 & 11056.7 \\
\hline 0 & 1 & 20 & 20 & -0.16672 & -0.16027 & -0.16027 & 0.00175 & -9524.7 & -9156.6 & -9156.5 \\
\hline 0 & 1 & 20 & 50 & -0.16794 & -0.16533 & -0.16533 & 0.00036 & -46074.3 & -45356.7 & -45356.0 \\
\hline 0 & 1 & 50 & 20 & -0.16893 & -0.16205 & -0.16206 & -0.00096 & 17560.3 & 16845.2 & 16845.6 \\
\hline 0 & 1 & 50 & 50 & -0.16840 & -0.16567 & -0.16567 & -0.00052 & 32078.4 & 31557.8 & 31558.2 \\
\hline 0.5 & 0.5 & 20 & 20 & -0.09493 & -0.04838 & -0.04839 & -0.00011 & 85619.9 & 43636.1 & 43649.3 \\
\hline 0.5 & 0.5 & 20 & 50 & -0.09909 & -0.05352 & -0.05352 & -0.01170 & 846.9 & 457.5 & 457.4 \\
\hline 0.5 & 0.5 & 50 & 20 & -0.09532 & -0.04728 & -0.04729 & 0.00071 & -13437.0 & -6665.1 & -6666.9 \\
\hline 0.5 & 0.5 & 50 & 50 & -0.09412 & -0.04779 & -0.04780 & -0.01000 & 941.1 & 477.8 & 477.9 \\
\hline 0.5 & 1 & 20 & 20 & $\overline{-0.19759}$ & -0.15950 & -0.15949 & -0.00223 & 8849.4 & 7143.6 & 7143.1 \\
\hline 0.5 & 1 & 20 & 50 & -0.19391 & -0.16041 & -0.16041 & 0.00171 & -11339.7 & -9380.3 & -9380.3 \\
\hline 0.5 & 1 & 50 & 20 & -0.19492 & -0.15553 & -0.15553 & 0.00231 & -8430.1 & -6726.7 & -6726.6 \\
\hline 0.5 & 1 & 50 & 50 & -0.19661 & -0.16143 & -0.16143 & 0.00032 & -61804.7 & -50746.8 & -50746.2 \\
\hline 1 & 0.5 & 20 & 20 & $\overline{-0.18469}$ & -0.03702 & -0.03699 & 0.00703 & $\begin{array}{c}-2626.3 \\
\end{array}$ & -526.4 & -526.0 \\
\hline 1 & 0.5 & 20 & 50 & -0.19261 & -0.04537 & -0.04537 & -0.00187 & 10272.8 & 2419.7 & 2419.6 \\
\hline 1 & 0.5 & 50 & 20 & -0.20071 & -0.05124 & -0.05124 & -0.00746 & 2689.4 & 686.6 & 686.6 \\
\hline 1 & 0.5 & 50 & 50 & -0.19721 & -0.04850 & -0.04847 & -0.00338 & 5839.4 & 1436.0 & 1435.3 \\
\hline 1 & 1 & 20 & 20 & $\overline{-0.27292}$ & -0.15831 & -0.15829 & -0.01247 & 2188.9 & 1269.7 & 1269.5 \\
\hline 1 & 1 & 20 & 50 & -0.26258 & -0.14970 & -0.14972 & 0.00184 & -14249.7 & -8124.0 & -8124.8 \\
\hline 1 & 1 & 50 & 20 & -0.26780 & -0.14897 & -0.14892 & -0.00129 & 20751.2 & 11543.0 & 11539.7 \\
\hline 1 & 1 & 50 & 50 & -0.26515 & -0.15080 & -0.15080 & 0.00131 & -20176.2 & -11474.9 & -11475.4 \\
\hline
\end{tabular}


Tabela B.5: Média dos EPs estimados de $\hat{\gamma}_{00}$ sob cada ajuste, quando $\gamma_{00}=0$.

\begin{tabular}{|c|c|c|c|c|c|c|c|c|c|c|c|c|}
\hline \multicolumn{4}{|c|}{ Valores fixados } & \multicolumn{5}{|c|}{ Média dos EPs } & \multicolumn{4}{|c|}{$\%$ relativa a QG3 } \\
\hline$\tau_{0}$ & $\sigma$ & $J$ & $n_{j}$ & MLG & $\mathrm{QV}$ & QGR & QG2 & QG3 & MLG & QV & QGR & QG2 \\
\hline 0 & 0 & 20 & 20 & 0.0224 & 0.0223 & 0.0227 & 0.0234 & 0.0235 & 95 & 95 & 97 & 99 \\
\hline 0 & 0 & 20 & 50 & 0.0141 & 0.0141 & 0.0144 & 0.0148 & 0.0149 & 95 & 95 & 97 & 99 \\
\hline 0 & 0 & 50 & 20 & 0.0141 & 0.0141 & 0.0145 & 0.0147 & 0.0148 & 96 & 96 & 98 & 99 \\
\hline 0 & 0 & 50 & 50 & 0.0089 & 0.0089 & 0.0091 & 0.0093 & 0.0093 & 96 & 96 & 98 & 100 \\
\hline 0.5 & 0 & 20 & 20 & 0.0224 & 0.0316 & 0.1125 & 0.1097 & 0.1079 & 21 & 29 & 104 & 102 \\
\hline 0.5 & 0 & 20 & 50 & 0.0142 & 0.0200 & 0.1115 & 0.1082 & 0.1112 & 13 & 18 & 100 & 97 \\
\hline 0.5 & 0 & 50 & 20 & 0.0142 & 0.0201 & 0.0721 & 0.0708 & 0.0702 & 20 & 29 & 103 & 101 \\
\hline 0.5 & 0 & 50 & 50 & 0.0090 & 0.0128 & 0.0716 & 0.0703 & 0.0763 & 12 & 17 & 94 & 92 \\
\hline 1 & 0 & 20 & 20 & 0.0225 & 0.0455 & 0.2254 & 0.2156 & 0.2158 & 10 & 21 & 104 & 100 \\
\hline 1 & 0 & 20 & 50 & 0.0142 & 0.0289 & 0.2257 & 0.2165 & 0.2163 & 7 & 13 & 104 & 100 \\
\hline 1 & 0 & 50 & 20 & 0.0142 & 0.0292 & 0.1452 & 0.1407 & 0.1409 & 10 & 21 & 103 & 100 \\
\hline 1 & 0 & 50 & 50 & 0.0090 & 0.0184 & 0.1435 & 0.1398 & 0.1401 & 6 & 13 & 102 & 100 \\
\hline 0 & 0.5 & 20 & 20 & 0.0224 & 0.0321 & 0.0313 & 0.0312 & 0.0355 & 63 & 90 & 88 & 88 \\
\hline 0 & 0.5 & 20 & 50 & 0.0141 & 0.0203 & 0.0196 & 0.0196 & 0.0225 & 63 & 90 & 87 & 87 \\
\hline 0 & 0.5 & 50 & 20 & 0.0141 & 0.0203 & 0.0200 & 0.0200 & 0.0222 & 64 & 92 & 90 & 90 \\
\hline 0 & 0.5 & 50 & 50 & 0.0089 & 0.0128 & 0.0127 & 0.0127 & 0.0141 & 64 & 91 & 90 & 90 \\
\hline 0 & 1 & 20 & 20 & 0.0224 & 0.0464 & 0.0450 & 0.0447 & 0.0584 & 38 & 79 & 77 & 77 \\
\hline 0 & 1 & 20 & 50 & 0.0141 & 0.0293 & 0.0285 & 0.0284 & 0.0370 & 38 & 79 & 77 & 77 \\
\hline 0 & 1 & 50 & 20 & 0.0141 & 0.0293 & 0.0293 & 0.0291 & 0.0365 & 39 & 80 & 80 & 80 \\
\hline 0 & 1 & 50 & 50 & 0.0089 & 0.0185 & 0.0184 & 0.0183 & 0.0231 & 39 & 80 & 79 & 79 \\
\hline 0.5 & 0.5 & 20 & 20 & 0.0224 & 0.0380 & 0.1091 & 0.1063 & 0.1119 & 20 & 34 & 97 & 95 \\
\hline 0.5 & 0.5 & 20 & 50 & 0.0142 & 0.0240 & 0.1068 & 0.1036 & 0.1075 & 13 & 22 & 99 & 96 \\
\hline 0.5 & 0.5 & 50 & 20 & 0.0142 & 0.0241 & 0.0702 & 0.0690 & 0.0727 & 19 & 33 & 97 & 95 \\
\hline 0.5 & 0.5 & 50 & 50 & 0.0089 & 0.0152 & 0.0679 & 0.0668 & 0.0702 & 13 & 22 & 97 & 95 \\
\hline 0.5 & 1 & 20 & 20 & 0.0224 & 0.0491 & 0.1025 & 0.0999 & 0.1194 & 19 & 41 & 86 & 84 \\
\hline 0.5 & 1 & 20 & 50 & 0.0142 & 0.0311 & 0.0965 & 0.0939 & 0.1126 & 13 & 28 & 86 & 83 \\
\hline 0.5 & 1 & 50 & 20 & 0.0141 & 0.0311 & 0.0664 & 0.0653 & 0.0779 & 18 & 40 & 85 & 84 \\
\hline 0.5 & 1 & 50 & 50 & 0.0089 & 0.0197 & 0.0618 & 0.0608 & 0.0729 & 12 & 27 & 85 & 83 \\
\hline 1 & 0.5 & 20 & 20 & 0.0225 & 0.0488 & 0.2179 & 0.2088 & 0.2186 & 10 & 22 & 100 & 96 \\
\hline 1 & 0.5 & 20 & 50 & 0.0142 & 0.0308 & 0.2135 & 0.2047 & 0.2140 & 7 & 14 & 100 & 96 \\
\hline 1 & 0.5 & 50 & 20 & 0.0142 & 0.0310 & 0.1394 & 0.1350 & 0.1415 & 10 & 22 & 99 & 95 \\
\hline 1 & 0.5 & 50 & 50 & 0.0090 & 0.0196 & 0.1370 & 0.1336 & 0.1401 & 6 & 14 & 98 & 95 \\
\hline 1 & 1 & 20 & 20 & 0.0224 & 0.0556 & 0.1979 & 0.1901 & 0.2241 & 10 & 25 & 88 & 85 \\
\hline 1 & 1 & 20 & 50 & 0.0142 & 0.0350 & 0.1907 & 0.1833 & 0.2171 & 7 & 16 & 88 & 84 \\
\hline 1 & 1 & 50 & 20 & 0.0142 & 0.0351 & 0.1254 & 0.1217 & 0.1436 & 10 & 24 & 87 & 85 \\
\hline 1 & 1 & 50 & 50 & 0.0090 & 0.0222 & 0.1223 & 0.1191 & 0.1412 & 6 & 16 & 87 & 84 \\
\hline
\end{tabular}


Tabela B.6: Média dos EPs estimados de $\hat{\gamma}_{00}$ sob cada ajuste, quando $\gamma_{00}=1$.

\begin{tabular}{|c|c|c|c|c|c|c|c|c|c|c|c|c|}
\hline \multicolumn{4}{|c|}{ Valores fixados } & \multicolumn{5}{|c|}{ Média dos EPs } & \multicolumn{4}{|c|}{$\%$ relativa a QG3 } \\
\hline$\tau_{0}$ & $\sigma$ & $J$ & $n_{j}$ & MLG & QV & QGR & QG2 & QG3 & MLG & QV & QGR & QG2 \\
\hline 0 & 0 & 20 & 20 & 0.0252 & 0.0252 & 0.0258 & 0.0264 & 0.0268 & 94 & 94 & 96 & 99 \\
\hline 0 & 0 & 20 & 50 & 0.0159 & 0.0159 & 0.0162 & 0.0167 & 0.0168 & 95 & 95 & 96 & 99 \\
\hline 0 & 0 & 50 & 20 & 0.0159 & 0.0159 & 0.0162 & 0.0165 & 0.0167 & 96 & 96 & 97 & 99 \\
\hline 0 & 0 & 50 & 50 & 0.0101 & 0.0101 & 0.0103 & 0.0105 & 0.0105 & 96 & 96 & 98 & 99 \\
\hline 0.5 & 0 & 20 & 20 & 0.0250 & 0.0338 & 0.1135 & 0.1106 & 0.1104 & 23 & 31 & 103 & 100 \\
\hline 0.5 & 0 & 20 & 50 & 0.0158 & 0.0214 & 0.1124 & 0.1094 & 0.1134 & 14 & 19 & 99 & 96 \\
\hline 0.5 & 0 & 50 & 20 & 0.0158 & 0.0215 & 0.0732 & 0.0718 & 0.0723 & 22 & 30 & 101 & 99 \\
\hline 0.5 & 0 & 50 & 50 & 0.0100 & 0.0136 & 0.0716 & 0.0704 & 0.0788 & 13 & 17 & 91 & 89 \\
\hline 1 & 0 & 20 & 20 & 0.0244 & 0.0476 & 0.2267 & 0.2169 & 0.2167 & 11 & 22 & 105 & 100 \\
\hline 1 & 0 & 20 & 50 & 0.0155 & 0.0301 & 0.2246 & 0.2156 & 0.2163 & 7 & 14 & 104 & 100 \\
\hline 1 & 0 & 50 & 20 & 0.0154 & 0.0305 & 0.1454 & 0.1405 & 0.1407 & 11 & 22 & 103 & 100 \\
\hline 1 & 0 & 50 & 50 & 0.0097 & 0.0193 & 0.1433 & 0.1395 & 0.1400 & 7 & 14 & 102 & 100 \\
\hline 0 & 0.5 & 20 & 20 & 0.0249 & 0.0342 & 0.0333 & 0.0333 & 0.0380 & 65 & 90 & 88 & 87 \\
\hline 0 & 0.5 & 20 & 50 & 0.0158 & 0.0216 & 0.0210 & 0.0210 & 0.0241 & 65 & 90 & 87 & 87 \\
\hline 0 & 0.5 & 50 & 20 & 0.0158 & 0.0216 & 0.0214 & 0.0214 & 0.0237 & 67 & 91 & 90 & 90 \\
\hline 0 & 0.5 & 50 & 50 & 0.0100 & 0.0137 & 0.0135 & 0.0135 & 0.0150 & 66 & 91 & 90 & 90 \\
\hline 0 & 1 & 20 & 20 & 0.0243 & 0.0487 & 0.0470 & 0.0468 & 0.0602 & 40 & 81 & 78 & 78 \\
\hline 0 & 1 & 20 & 50 & 0.0154 & 0.0308 & 0.0298 & 0.0297 & 0.0382 & 40 & 81 & 78 & 78 \\
\hline 0 & 1 & 50 & 20 & 0.0154 & 0.0308 & 0.0307 & 0.0306 & 0.0378 & 41 & 81 & 81 & 81 \\
\hline 0 & 1 & 50 & 50 & 0.0097 & 0.0195 & 0.0193 & 0.0192 & 0.0239 & 41 & 81 & 81 & 81 \\
\hline 0.5 & 0.5 & 20 & 20 & 0.0247 & 0.0401 & 0.1117 & 0.1088 & 0.1139 & 22 & 35 & 98 & 96 \\
\hline 0.5 & 0.5 & 20 & 50 & 0.0156 & 0.0254 & 0.1089 & 0.1059 & 0.1097 & 14 & 23 & 99 & 97 \\
\hline 0.5 & 0.5 & 50 & 20 & 0.0156 & 0.0255 & 0.0717 & 0.0704 & 0.0737 & 21 & 35 & 97 & 96 \\
\hline 0.5 & 0.5 & 50 & 50 & 0.0099 & 0.0161 & 0.0692 & 0.0680 & 0.0715 & 14 & 23 & 97 & 95 \\
\hline 0.5 & 1 & 20 & 20 & 0.0242 & 0.0515 & 0.1062 & 0.1036 & 0.1217 & 20 & 42 & 87 & 85 \\
\hline 0.5 & 1 & 20 & 50 & 0.0153 & 0.0325 & 0.0978 & 0.0952 & 0.1122 & 14 & 29 & 87 & 85 \\
\hline 0.5 & 1 & 50 & 20 & 0.0153 & 0.0326 & 0.0682 & 0.0670 & 0.0786 & 19 & 42 & 87 & 85 \\
\hline 0.5 & 1 & 50 & 50 & 0.0097 & 0.0206 & 0.0633 & 0.0622 & 0.0732 & 13 & 28 & 86 & 85 \\
\hline 1 & 0.5 & 20 & 20 & 0.0244 & 0.0508 & 0.2186 & 0.2091 & 0.2176 & 11 & 23 & 100 & 96 \\
\hline 1 & 0.5 & 20 & 50 & 0.0154 & 0.0323 & 0.2180 & 0.2090 & 0.2171 & 7 & 15 & 100 & 96 \\
\hline 1 & 0.5 & 50 & 20 & 0.0153 & 0.0325 & 0.1404 & 0.1358 & 0.1416 & 11 & 23 & 99 & 96 \\
\hline 1 & 0.5 & 50 & 50 & 0.0097 & 0.0205 & 0.1380 & 0.1343 & 0.1402 & 7 & 15 & 98 & 96 \\
\hline 1 & 1 & 20 & 20 & 0.0239 & 0.0578 & 0.1999 & 0.1917 & 0.2230 & 11 & 26 & 90 & 86 \\
\hline 1 & 1 & 20 & 50 & 0.0152 & 0.0366 & 0.1948 & 0.1867 & 0.2173 & 7 & 17 & 90 & 86 \\
\hline 1 & 1 & 50 & 20 & 0.0151 & 0.0367 & 0.1283 & 0.1241 & 0.1444 & 10 & 25 & 89 & 86 \\
\hline 1 & 1 & 50 & 50 & 0.0096 & 0.0232 & 0.1237 & 0.1204 & 0.1407 & 7 & 16 & 88 & 86 \\
\hline
\end{tabular}


Tabela B.7: DP das estimativas de $\gamma_{00}$ sob cada ajuste, quando $\gamma_{00}=0$.

\begin{tabular}{|c|c|c|c|c|c|c|c|c|c|c|}
\hline \multicolumn{4}{|c|}{ Valores fixados } & \multicolumn{4}{|c|}{ DPs } & \multicolumn{3}{|c|}{$\%$ relativa a QG3 } \\
\hline$\tau_{0}$ & $\sigma$ & $J$ & $n_{j}$ & MLG* $^{*}$ & QGR & QG2 & QG3 & MLG & QGR & QG2 \\
\hline 0 & 0 & 20 & 20 & $\overline{0.02253}$ & 0.02253 & 0.02254 & 0.02256 & 99.9 & 99.9 & 99.9 \\
\hline 0 & 0 & 20 & 50 & 0.01398 & 0.01398 & 0.01397 & 0.01399 & 99.9 & 99.9 & 99.9 \\
\hline 0 & 0 & 50 & 20 & 0.01414 & 0.01415 & 0.01414 & 0.01416 & 99.9 & 99.9 & 99.9 \\
\hline 0 & 0 & 50 & 50 & 0.00886 & 0.00886 & 0.00885 & 0.00887 & 99.9 & 99.9 & 99.8 \\
\hline 0.5 & 0 & 20 & 20 & $\overline{0.10717}$ & 0.11314 & 0.11314 & 0.11309 & $\overline{94.8}$ & 100.1 & 100.0 \\
\hline 0.5 & 0 & 20 & 50 & 0.10763 & 0.11376 & 0.11379 & 0.11761 & 91.5 & 96.7 & 96.7 \\
\hline 0.5 & 0 & 50 & 20 & 0.06805 & 0.07177 & 0.07178 & 0.07070 & 96.2 & 101.5 & 101.5 \\
\hline 0.5 & 0 & 50 & 50 & 0.06865 & 0.07272 & 0.07272 & 0.07540 & 91.0 & 96.4 & 96.4 \\
\hline 1 & 0 & 20 & 20 & $\overline{0.19218}$ & 0.22818 & 0.22816 & 0.22815 & 84.2 & 100.0 & 100.0 \\
\hline 1 & 0 & 20 & 50 & 0.19262 & 0.22795 & 0.22795 & 0.22619 & 85.2 & 100.8 & 100.8 \\
\hline 1 & 0 & 50 & 20 & 0.11858 & 0.14157 & 0.14159 & 0.14166 & 83.7 & 99.9 & 99.9 \\
\hline 1 & 0 & 50 & 50 & 0.11675 & 0.13966 & 0.13968 & 0.13961 & 83.6 & 100.0 & 100.0 \\
\hline 0 & 0.5 & 20 & 20 & $\overline{0.03125}$ & 0.03132 & 0.03132 & 0.03307 & $\overline{94.5}$ & 94.7 & 94.7 \\
\hline 0 & 0.5 & 20 & 50 & 0.02001 & 0.02002 & 0.02003 & 0.02118 & 94.5 & 94.5 & 94.6 \\
\hline 0 & 0.5 & 50 & 20 & 0.02050 & 0.02055 & 0.02056 & 0.02172 & 94.4 & 94.6 & 94.6 \\
\hline 0 & 0.5 & 50 & 50 & 0.01298 & 0.01299 & 0.01299 & 0.01377 & 94.3 & 94.4 & 94.4 \\
\hline$\overline{0}$ & 1 & 20 & 20 & $\overline{0.04470}$ & 0.04502 & 0.04502 & 0.05431 & 82.3 & 82.9 & 82.9 \\
\hline 0 & 1 & 20 & 50 & 0.02956 & 0.02965 & 0.02965 & 0.03578 & 82.6 & 82.9 & 82.9 \\
\hline 0 & 1 & 50 & 20 & 0.02931 & 0.02955 & 0.02955 & 0.03537 & 82.9 & 83.5 & 83.5 \\
\hline 0 & 1 & 50 & 50 & 0.01845 & 0.01851 & 0.01852 & 0.02226 & 82.9 & 83.2 & 83.2 \\
\hline 0.5 & 0.5 & 20 & 20 & $\overline{0.10446}$ & 0.10970 & 0.10970 & 0.11562 & $\overline{90.4}$ & 94.9 & 94.9 \\
\hline 0.5 & 0.5 & 20 & 50 & 0.10466 & 0.11002 & 0.11004 & 0.11667 & 89.7 & 94.3 & 94.3 \\
\hline 0.5 & 0.5 & 50 & 20 & 0.06544 & 0.06902 & 0.06902 & 0.07268 & 90.0 & 95.0 & 95.0 \\
\hline 0.5 & 0.5 & 50 & 50 & 0.06760 & 0.07098 & 0.07099 & 0.07438 & 90.9 & 95.4 & 95.4 \\
\hline 0.5 & 1 & 20 & 20 & $\overline{0.09944}$ & 0.10422 & 0.10422 & 0.12449 & $\overline{79.9}$ & 83.7 & 83.7 \\
\hline 0.5 & 1 & 20 & 50 & 0.09592 & 0.09997 & 0.09998 & 0.11982 & 80.1 & 83.4 & 83.4 \\
\hline 0.5 & 1 & 50 & 20 & 0.06292 & 0.06597 & 0.06598 & 0.07854 & 80.1 & 84.0 & 84.0 \\
\hline 0.5 & 1 & 50 & 50 & 0.05722 & 0.05977 & 0.05978 & 0.07175 & 79.7 & 83.3 & 83.3 \\
\hline 1 & 0.5 & 20 & 20 & $\overline{0.18170}$ & 0.21479 & 0.21481 & 0.22503 & 80.7 & 95.5 & 95.5 \\
\hline 1 & 0.5 & 20 & 50 & 0.18544 & 0.21784 & 0.21790 & 0.22805 & 81.3 & 95.5 & 95.5 \\
\hline 1 & 0.5 & 50 & 20 & 0.11404 & 0.13523 & 0.13525 & 0.14169 & 80.5 & 95.4 & 95.5 \\
\hline 1 & 0.5 & 50 & 50 & 0.11536 & 0.13639 & 0.13646 & 0.14305 & 80.6 & 95.3 & 95.4 \\
\hline 1 & 1 & 20 & 20 & $\overline{0.16872}$ & 0.19557 & 0.19562 & 0.23070 & $\overline{73.1}$ & 84.8 & 84.8 \\
\hline 1 & 1 & 20 & 50 & 0.16104 & 0.18460 & 0.18460 & 0.21793 & 73.9 & 84.7 & 84.7 \\
\hline 1 & 1 & 50 & 20 & 0.10778 & 0.12500 & 0.12503 & 0.14723 & 73.2 & 84.9 & 84.9 \\
\hline 1 & 1 & 50 & 50 & 0.10114 & 0.11618 & 0.11620 & 0.13788 & 73.4 & 84.3 & 84.3 \\
\hline
\end{tabular}

* Idem para QV 
Tabela B.8: DP das estimativas de $\gamma_{00}$ sob cada ajuste, quando $\gamma_{00}=1$.

\begin{tabular}{|c|c|c|c|c|c|c|c|c|c|c|}
\hline \multicolumn{4}{|c|}{ Valores fixados } & \multicolumn{4}{|c|}{ DPs } & \multicolumn{3}{|c|}{$\%$ relativa a QG3 } \\
\hline$\tau_{0}$ & $\sigma$ & $J$ & $n_{j}$ & MLG* & QGR & QG2 & QG3 & MLG & QGR & QG2 \\
\hline 0 & 0 & 20 & 20 & $\overline{0.02563}$ & 0.02566 & 0.02566 & 0.02581 & $\overline{99.3}$ & 99.4 & 99.4 \\
\hline 0 & 0 & 20 & 50 & 0.01546 & 0.01546 & 0.01547 & 0.01554 & 99.5 & 99.5 & 99.5 \\
\hline 0 & 0 & 50 & 20 & 0.01567 & 0.01568 & 0.01568 & 0.01586 & 98.9 & 98.9 & 98.9 \\
\hline 0 & 0 & 50 & 50 & 0.01008 & 0.01008 & 0.01008 & 0.01015 & 99.3 & 99.3 & 99.2 \\
\hline 0.5 & 0 & 20 & 20 & $\overline{0.11040}$ & 0.11409 & 0.11409 & 0.11508 & $\overline{95.9}$ & 99.1 & 99.1 \\
\hline 0.5 & 0 & 20 & 50 & 0.10853 & 0.11256 & 0.11256 & 0.11727 & 92.5 & 96.0 & 96.0 \\
\hline 0.5 & 0 & 50 & 20 & 0.06977 & 0.07242 & 0.07243 & 0.07380 & 94.5 & 98.1 & 98.1 \\
\hline 0.5 & 0 & 50 & 50 & 0.06957 & 0.07228 & 0.07229 & 0.07524 & 92.5 & 96.1 & 96.1 \\
\hline 1 & 0 & 20 & 20 & $\overline{0.19432}$ & 0.22382 & 0.22380 & 0.22421 & 86.7 & 99.8 & 99.8 \\
\hline 1 & 0 & 20 & 50 & 0.20108 & 0.23063 & 0.23060 & 0.23124 & 87.0 & 99.7 & 99.7 \\
\hline 1 & 0 & 50 & 20 & 0.12383 & 0.14330 & 0.14329 & 0.14352 & 86.3 & 99.8 & 99.8 \\
\hline 1 & 0 & 50 & 50 & 0.11916 & 0.13715 & 0.13712 & 0.13710 & 86.9 & 100.0 & 100.0 \\
\hline 0 & 0.5 & 20 & 20 & $\overline{0.03379}$ & 0.03387 & 0.03387 & 0.03588 & $\overline{94.2}$ & 94.4 & 94.4 \\
\hline 0 & 0.5 & 20 & 50 & 0.02208 & 0.02208 & 0.02208 & 0.02353 & 93.8 & 93.8 & 93.8 \\
\hline 0 & 0.5 & 50 & 20 & 0.02113 & 0.02122 & 0.02122 & 0.02261 & 93.5 & 93.9 & 93.9 \\
\hline 0 & 0.5 & 50 & 50 & 0.01392 & 0.01393 & 0.01394 & 0.01487 & 93.6 & 93.7 & 93.8 \\
\hline 0 & 1 & 20 & 20 & $\overline{0.04661}$ & 0.04710 & 0.04711 & 0.05581 & $\overline{83.5}$ & 84.4 & 84.4 \\
\hline 0 & 1 & 20 & 50 & 0.03104 & 0.03116 & 0.03117 & 0.03682 & 84.3 & 84.6 & 84.6 \\
\hline 0 & 1 & 50 & 20 & 0.03037 & 0.03065 & 0.03066 & 0.03608 & 84.2 & 85.0 & 85.0 \\
\hline 0 & 1 & 50 & 50 & 0.01968 & 0.01973 & 0.01974 & 0.02320 & 84.9 & 85.1 & 85.1 \\
\hline 0.5 & 0.5 & 20 & 20 & $\overline{0.10746}$ & 0.11104 & 0.11104 & 0.11622 & $\overline{92.5}$ & 95.5 & 95.5 \\
\hline 0.5 & 0.5 & 20 & 50 & 0.10180 & 0.10582 & 0.10582 & 0.11264 & 90.4 & 93.9 & 93.9 \\
\hline 0.5 & 0.5 & 50 & 20 & 0.06972 & 0.07265 & 0.07265 & 0.07599 & 91.7 & 95.6 & 95.6 \\
\hline 0.5 & 0.5 & 50 & 50 & 0.06887 & 0.07151 & 0.07149 & 0.07687 & 89.6 & 93.0 & 93.0 \\
\hline 0.5 & 1 & 20 & 20 & $\overline{0.10035}$ & 0.10438 & 0.10440 & 0.12244 & 82.0 & 85.3 & 85.3 \\
\hline 0.5 & 1 & 20 & 50 & 0.09655 & 0.09973 & 0.09974 & 0.11786 & 81.9 & 84.6 & 84.6 \\
\hline 0.5 & 1 & 50 & 20 & 0.06598 & 0.06913 & 0.06914 & 0.08097 & 81.5 & 85.4 & 85.4 \\
\hline 0.5 & 1 & 50 & 50 & 0.06133 & 0.06389 & 0.06391 & 0.07560 & 81.1 & 84.5 & 84.5 \\
\hline 1 & 0.5 & 20 & 20 & $\overline{0.18974}$ & 0.21744 & 0.21746 & 0.22674 & $\overline{83.7}$ & 95.9 & 95.9 \\
\hline 1 & 0.5 & 20 & 50 & 0.18122 & 0.20619 & 0.20622 & 0.21558 & 84.1 & 95.6 & 95.7 \\
\hline 1 & 0.5 & 50 & 20 & 0.12026 & 0.13868 & 0.13869 & 0.14464 & 83.1 & 95.9 & 95.9 \\
\hline 1 & 0.5 & 50 & 50 & 0.11680 & 0.13444 & 0.13443 & 0.14033 & 83.2 & 95.8 & 95.8 \\
\hline 1 & 1 & 20 & 20 & $\overline{0.17369}$ & 0.19890 & 0.19889 & 0.23025 & $\overline{75.4}$ & 86.4 & 86.4 \\
\hline 1 & 1 & 20 & 50 & 0.17594 & 0.20043 & 0.20050 & 0.23382 & 75.2 & 85.7 & 85.8 \\
\hline 1 & 1 & 50 & 20 & 0.10774 & 0.12362 & 0.12358 & 0.14358 & 75.0 & 86.1 & 86.1 \\
\hline 1 & 1 & 50 & 50 & 0.10665 & 0.12194 & 0.12195 & 0.14254 & 74.8 & 85.5 & 85.6 \\
\hline
\end{tabular}

* Idem para QV 
Tabela B.9: Taxa de rejeição (\%) estimada para $H_{0}: \gamma_{00}=0$ vs $H_{a}: \gamma_{00} \neq 0$.

\begin{tabular}{|c|c|c|c|c|c|c|c|c|}
\hline \multicolumn{4}{|c|}{ Valores fixados } & \multicolumn{5}{|c|}{ Taxa de rejeição (\%) estimada } \\
\hline$\tau_{0}$ & $\sigma$ & $J$ & $n_{j}$ & MLG & QV & QGR & QG2 & QG3 \\
\hline 0 & 0 & 20 & 20 & 5.0 & 4.9 & 5.4 & 4.6 & 4.3 \\
\hline 0 & 0 & 20 & 50 & 4.4 & 4.4 & 4.7 & 4.2 & 4.3 \\
\hline 0 & 0 & 50 & 20 & 4.5 & 4.5 & 4.3 & 4.0 & 4.0 \\
\hline 0 & 0 & 50 & 50 & 4.4 & 4.6 & 4.4 & 3.9 & 3.8 \\
\hline 0.5 & 0 & 20 & 20 & 69.0 & 56.4 & 6.9 & 7.5 & 7.4 \\
\hline 0.5 & 0 & 20 & 50 & 78.4 & 70.1 & 7.4 & 8.2 & 9.1 \\
\hline 0.5 & 0 & 50 & 20 & 68.0 & 56.2 & 4.5 & 5.4 & 5.4 \\
\hline 0.5 & 0 & 50 & 50 & 80.0 & 70.9 & 4.9 & 5.3 & 5.7 \\
\hline 1 & 0 & 20 & 20 & 82.1 & 63.3 & 7.1 & 8.5 & 8.4 \\
\hline 1 & 0 & 20 & 50 & 87.2 & 75.7 & 6.4 & 7.1 & 6.6 \\
\hline 1 & 0 & 50 & 20 & 82.0 & 63.3 & 5.1 & 5.5 & 5.4 \\
\hline 1 & 0 & 50 & 50 & 88.5 & 76.1 & 4.9 & 5.5 & 5.3 \\
\hline 0 & 0.5 & 20 & 20 & 16.2 & 4.6 & 5.8 & 6.2 & 4.0 \\
\hline 0 & 0.5 & 20 & 50 & 16.2 & 4.8 & 6.5 & 6.6 & 3.7 \\
\hline 0 & 0.5 & 50 & 20 & 18.1 & 5.1 & 6.3 & 6.4 & 4.6 \\
\hline 0 & 0.5 & 50 & 50 & 17.8 & 5.9 & 7.1 & 6.9 & 5.4 \\
\hline 0 & 1 & 20 & 20 & 33.4 & 4.1 & 6.8 & 6.9 & 3.9 \\
\hline 0 & 1 & 20 & 50 & 35.3 & 5.2 & 6.4 & 6.4 & 4.6 \\
\hline 0 & 1 & 50 & 20 & 32.8 & 5.5 & 6.4 & 6.5 & 5.1 \\
\hline 0 & 1 & 50 & 50 & 35.0 & 4.4 & 5.7 & 5.9 & 3.8 \\
\hline 0.5 & 0.5 & 20 & 20 & 68.0 & 48.3 & 6.0 & 6.3 & 6.3 \\
\hline 0.5 & 0.5 & 20 & 50 & 78.8 & 63.8 & 7.1 & 7.6 & 8.6 \\
\hline 0.5 & 0.5 & 50 & 20 & 67.8 & 47.5 & 4.7 & 5.2 & 5.2 \\
\hline 0.5 & 0.5 & 50 & 50 & 79.0 & 64.7 & 6.9 & 7.6 & 7.6 \\
\hline 0.5 & 1 & 20 & 20 & 65.6 & 33.8 & 7.0 & 7.1 & 7.1 \\
\hline 0.5 & 1 & 20 & 50 & 76.6 & 52.3 & 8.0 & 8.7 & 8.8 \\
\hline 0.5 & 1 & 50 & 20 & 66.0 & 33.6 & 5.1 & 5.8 & 5.4 \\
\hline 0.5 & 1 & 50 & 50 & 75.7 & 49.9 & 3.8 & 4.2 & 4.0 \\
\hline 1 & 0.5 & 20 & 20 & 78.7 & 58.6 & 5.9 & 6.3 & 6.3 \\
\hline 1 & 0.5 & 20 & 50 & 88.1 & 74.4 & 6.5 & 7.8 & 7.7 \\
\hline 1 & 0.5 & 50 & 20 & 80.6 & 59.6 & 5.6 & 6.2 & 6.2 \\
\hline 1 & 0.5 & 50 & 50 & 86.5 & 72.4 & 5.5 & 6.4 & 6.4 \\
\hline 1 & 1 & 20 & 20 & 81.7 & 53.6 & 5.8 & 6.8 & 6.9 \\
\hline 1 & 1 & 20 & 50 & 87.4 & 68.4 & 5.7 & 6.5 & 6.4 \\
\hline 1 & 1 & 50 & 20 & 81.0 & 53.3 & 5.9 & 6.4 & 6.4 \\
\hline 1 & 1 & 50 & 50 & 85.5 & 67.3 & 4.4 & 5.1 & 5.2 \\
\hline
\end{tabular}


Tabela B.10: Taxa de rejeição (\%) estimada para $H_{0}: \gamma_{00}=1$ vs $H_{a}: \gamma_{00} \neq 1$.

\begin{tabular}{|c|c|c|c|c|c|c|c|c|}
\hline \multicolumn{4}{|c|}{ Valores fixados } & \multicolumn{5}{|c|}{ Taxa de rejeição (\%) estimada } \\
\hline$\tau_{0}$ & $\sigma$ & $J$ & $n_{j}$ & MLG & QV & QGR & QG2 & QG3 \\
\hline 0 & 0 & 20 & 20 & 5.8 & 6.1 & 5.6 & 4.6 & 4.6 \\
\hline 0 & 0 & 20 & 50 & 4.0 & 3.9 & 3.7 & 3.3 & 3.3 \\
\hline 0 & 0 & 50 & 20 & 3.9 & 4.0 & 3.8 & 3.6 & 3.7 \\
\hline 0 & 0 & 50 & 50 & 4.6 & 4.7 & 4.7 & 4.5 & 4.0 \\
\hline 0.5 & 0 & 20 & 20 & 68.9 & 58.7 & 6.1 & 6.4 & 6.4 \\
\hline 0.5 & 0 & 20 & 50 & 77.3 & 72.4 & 6.2 & 6.5 & 10.1 \\
\hline 0.5 & 0 & 50 & 20 & 75.4 & 65.8 & 5.3 & 5.3 & 6.2 \\
\hline 0.5 & 0 & 50 & 50 & 82.3 & 77.5 & 6.6 & 7.1 & 8.3 \\
\hline 1 & 0 & 20 & 20 & 86.2 & 73.2 & 5.6 & 6.3 & 6.4 \\
\hline 1 & 0 & 20 & 50 & 89.9 & 84.4 & 7.0 & 7.9 & 7.2 \\
\hline 1 & 0 & 50 & 20 & 92.4 & 85.3 & 5.3 & 6.0 & 5.9 \\
\hline 1 & 0 & 50 & 50 & 95.4 & 92.1 & 5.3 & 5.5 & 5.3 \\
\hline 0 & 0.5 & 20 & 20 & 57.0 & 35.3 & 36.0 & 36.0 & 4.1 \\
\hline 0 & 0.5 & 20 & 50 & 82.5 & 67.3 & 66.5 & 66.2 & 4.5 \\
\hline 0 & 0.5 & 50 & 20 & 84.5 & 70.8 & 67.3 & 67.3 & 3.5 \\
\hline 0 & 0.5 & 50 & 50 & 99.0 & 97.1 & 96.9 & 97.0 & 5.0 \\
\hline 0 & 1 & 20 & 20 & 99.4 & 93.4 & 90.4 & 90.9 & 3.2 \\
\hline 0 & 1 & 20 & 50 & 100.0 & 100.0 & 99.8 & 99.8 & 4.1 \\
\hline 0 & 1 & 50 & 20 & 100.0 & 100.0 & 99.9 & 99.9 & 3.7 \\
\hline 0 & 1 & 50 & 50 & 100.0 & 100.0 & 100.0 & 100.0 & 4.7 \\
\hline 0.5 & 0.5 & 20 & 20 & 76.0 & 61.9 & 7.9 & 8.4 & 7.1 \\
\hline 0.5 & 0.5 & 20 & 50 & 84.0 & 74.8 & 9.0 & 9.8 & 7.8 \\
\hline 0.5 & 0.5 & 50 & 20 & 86.9 & 77.1 & 11.1 & 11.8 & 6.3 \\
\hline 0.5 & 0.5 & 50 & 50 & 89.6 & 84.1 & 13.0 & 13.8 & 7.0 \\
\hline 0.5 & 1 & 20 & 20 & 94.4 & 83.6 & 33.7 & 35.6 & 6.4 \\
\hline 0.5 & 1 & 20 & 50 & 95.9 & 91.7 & 37.7 & 39.0 & 8.3 \\
\hline 0.5 & 1 & 50 & 20 & 99.4 & 97.1 & 62.2 & 63.6 & 6.3 \\
\hline 0.5 & 1 & 50 & 50 & 99.9 & 99.6 & 71.8 & 72.7 & 6.2 \\
\hline 1 & 0.5 & 20 & 20 & 87.4 & 75.0 & 6.2 & 7.7 & 7.1 \\
\hline 1 & 0.5 & 20 & 50 & 92.5 & 84.7 & 5.9 & 6.5 & 5.8 \\
\hline 1 & 0.5 & 50 & 20 & 94.3 & 89.0 & 6.3 & 7.2 & 6.0 \\
\hline 1 & 0.5 & 50 & 50 & 97.2 & 93.6 & 6.2 & 7.3 & 5.1 \\
\hline 1 & 1 & 20 & 20 & $\begin{array}{l}93.1 \\
\end{array}$ & 82.1 & 13.8 & 15.3 & 7.2 \\
\hline 1 & 1 & 20 & 50 & 95.5 & 88.9 & 14.6 & 15.7 & 8.2 \\
\hline 1 & 1 & 50 & 20 & 98.4 & 95.8 & 21.2 & 22.8 & 5.4 \\
\hline 1 & 1 & 50 & 50 & 99.2 & 98.1 & 24.1 & 25.3 & 6.1 \\
\hline
\end{tabular}





\section{Apêndice C}

\section{Gráficos referentes a $\tau_{0}$ e $\sigma$}

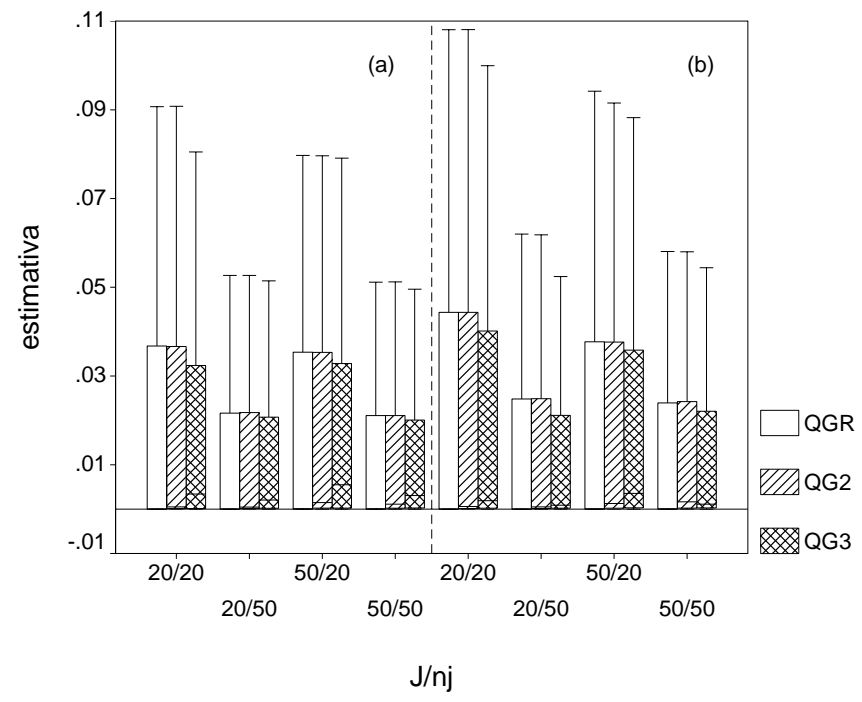

Figura C.1: Boxplots das estimativas de $\tau_{0}$ quando $\left(\gamma_{00}, \tau_{0}, \sigma\right)$ é igual a (a) $(0,0,0) ; \quad$ (b) $(1,0,0)$ 


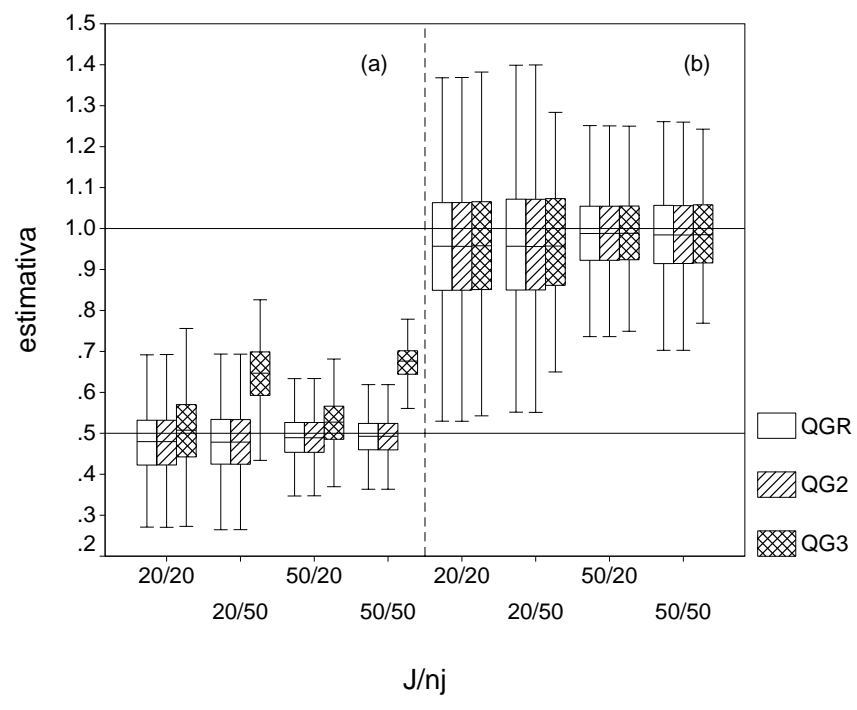

Figura C.2: Boxplots das estimativas de $\tau_{0}$ quando $\left(\gamma_{00}, \tau_{0}, \sigma\right)$ é igual a (a) $(0,0.5,0) ; \quad$ (b) $(0,1,0)$

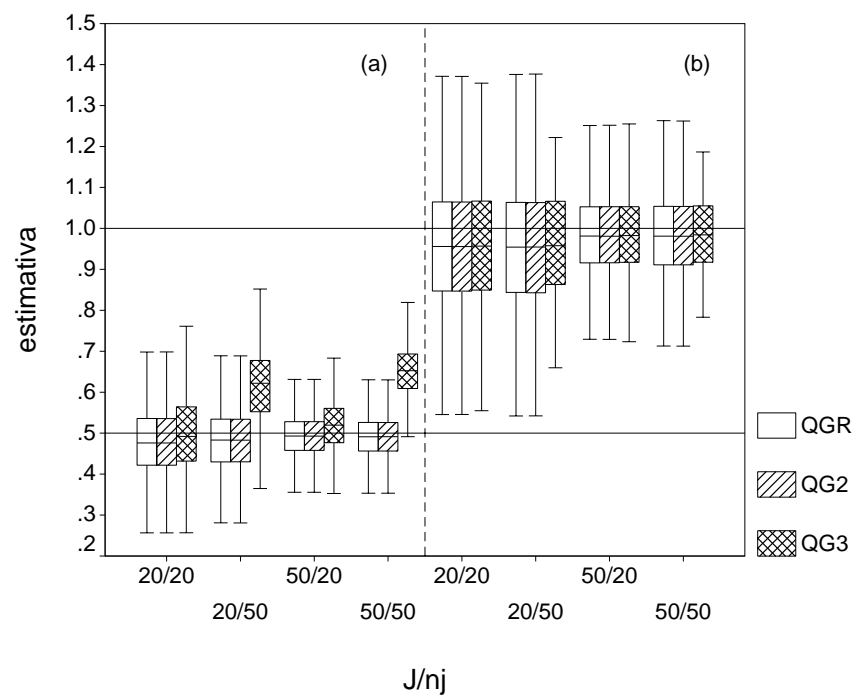

Figura C.3: Boxplots das estimativas de $\tau_{0}$ quando $\left(\gamma_{00}, \tau_{0}, \sigma\right)$ é igual a (a) $(1,0.5,0) ; \quad$ (b) $(1,1,0)$ 


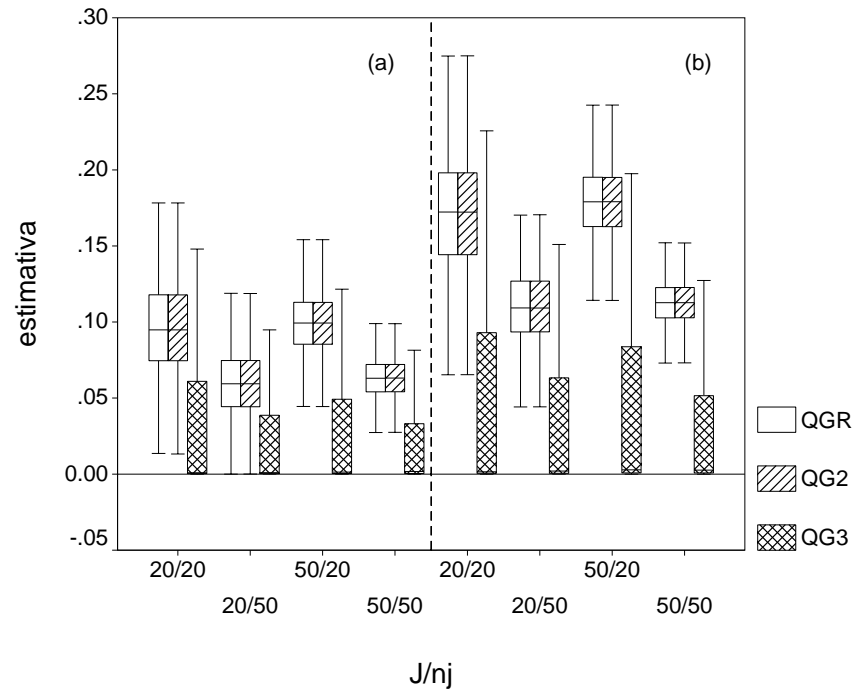

Figura C.4: Boxplots das estimativas de $\tau_{0}$ quando $\left(\gamma_{00}, \tau_{0}, \sigma\right)$ é igual a (a) $(0,0,0.5) ; \quad$ (b) $(0,0,1)$

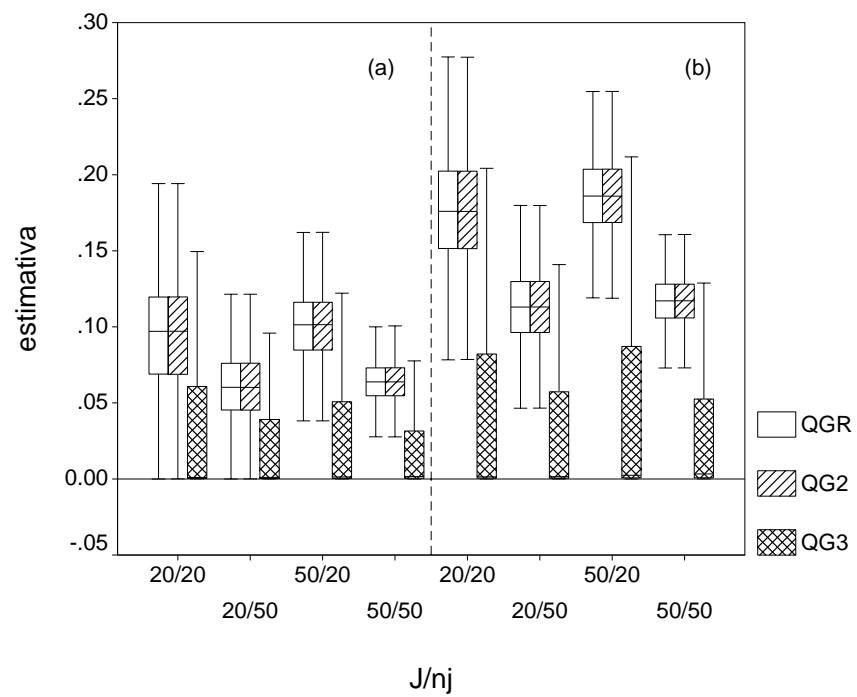

Figura C.5: Boxplots das estimativas de $\tau_{0}$ quando $\left(\gamma_{00}, \tau_{0}, \sigma\right)$ é igual a (a) $(1,0,0.5)$; (b) $(1,0,1)$ 


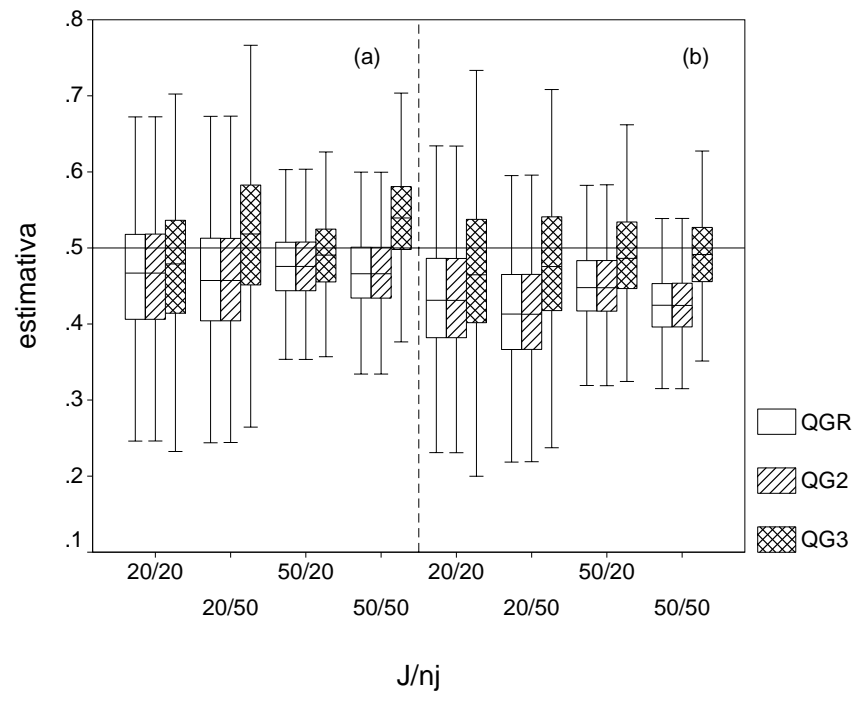

Figura C.6: Boxplots das estimativas de $\tau_{0}$ quando $\left(\gamma_{00}, \tau_{0}, \sigma\right)$ é igual a (a) $(0,0.5,0.5) ; \quad$ (b) $(0,0.5,1)$

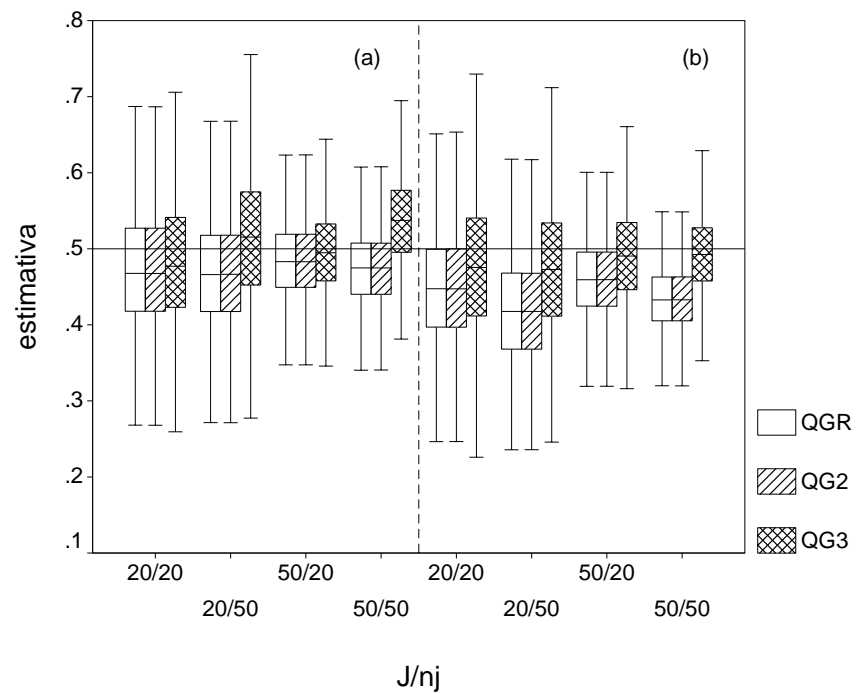

Figura C.7: Boxplots das estimativas de $\tau_{0}$ quando $\left(\gamma_{00}, \tau_{0}, \sigma\right)$ é igual a (a) $(1,0.5,0.5)$; (b) $(1,0.5,1)$ 


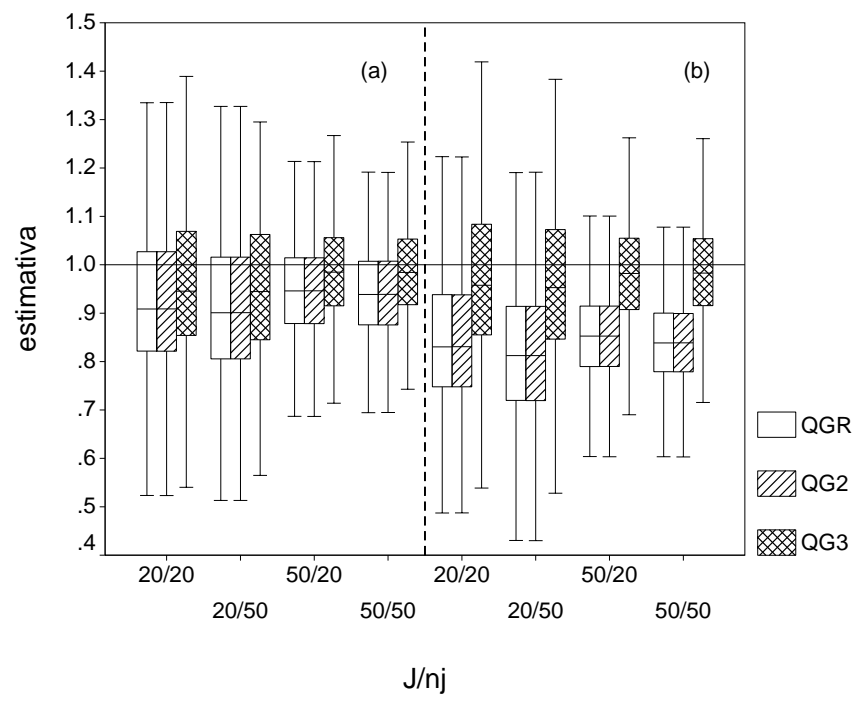

Figura C.8: Boxplots das estimativas de $\tau_{0}$ quando $\left(\gamma_{00}, \tau_{0}, \sigma\right)$ é igual a (a) $(0,1,0.5) ; \quad$ (b) $(0,1,1)$

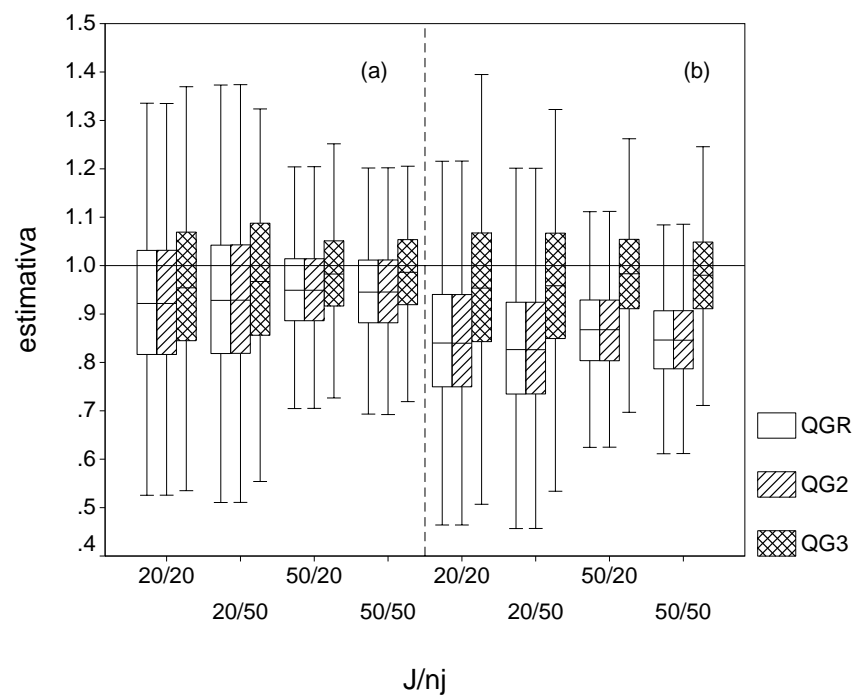

Figura C.9: Boxplots das estimativas de $\tau_{0}$ quando $\left(\gamma_{00}, \tau_{0}, \sigma\right)$ é igual a (a) $(1,1,0.5)$; (b) $(1,1,1)$ 


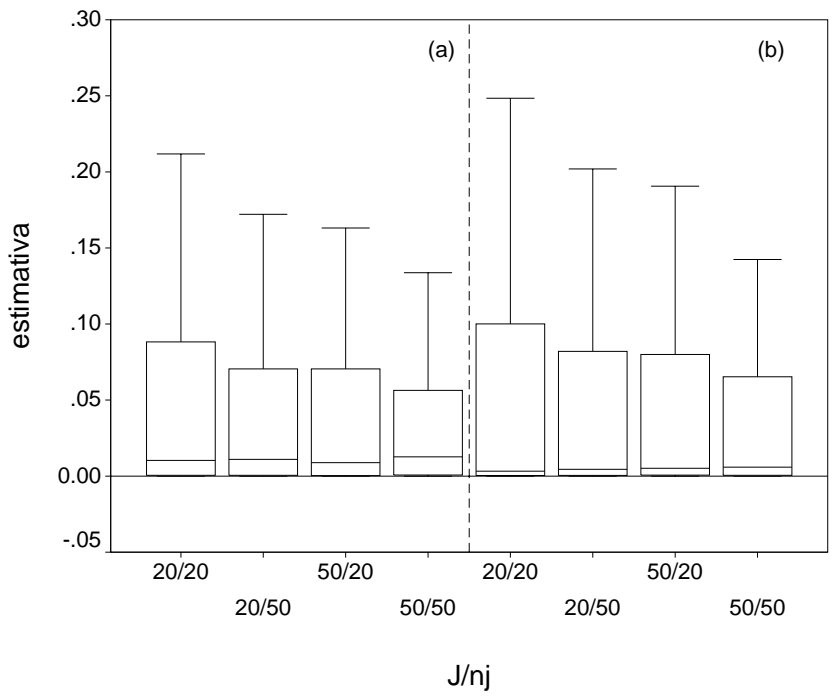

Figura C.10: Boxplots das estimativas de $\sigma$ quando $\left(\gamma_{00}, \tau_{0}, \sigma\right)$ é igual a (a) $(0,0,0) ; \quad$ (b) $(1,0,0)$ 


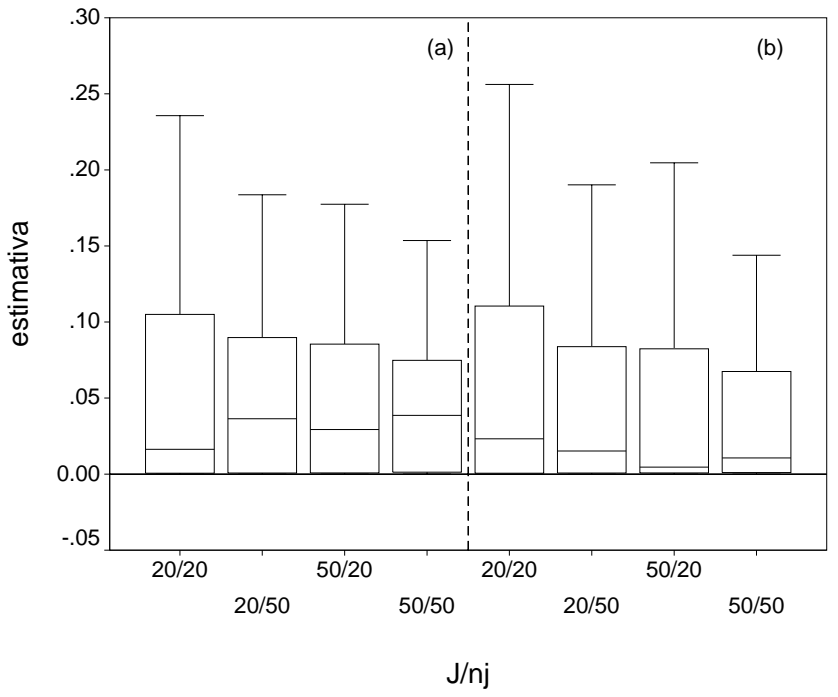

Figura C.11: Boxplots das estimativas de $\sigma$ quando $\left(\gamma_{00}, \tau_{0}, \sigma\right)$ é igual a (a) $(0,0.5,0) ; \quad$ (b) $(0,1,0)$

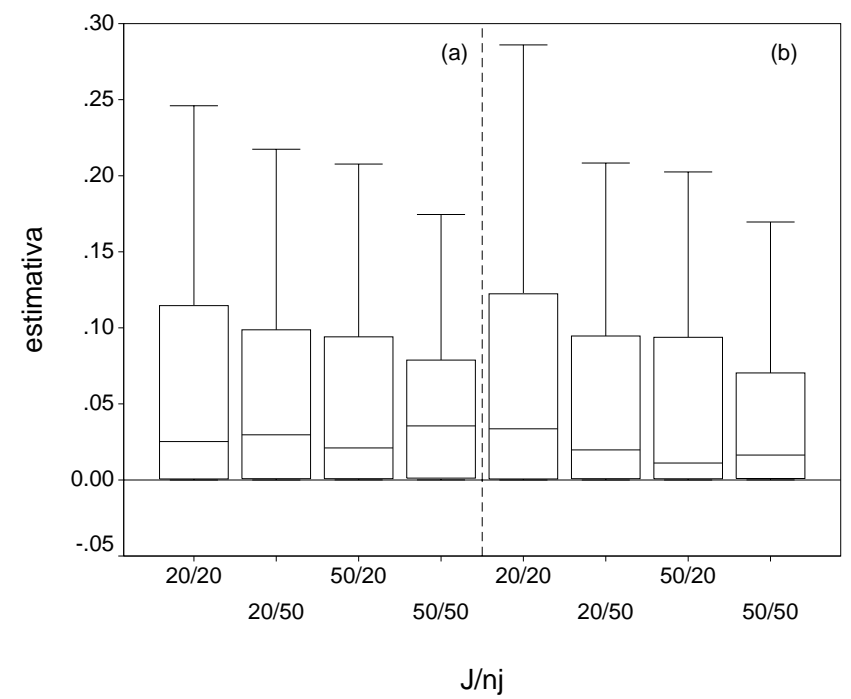

Figura C.12: Boxplots das estimativas de $\sigma$ quando $\left(\gamma_{00}, \tau_{0}, \sigma\right)$ é igual a (a) $(1,0.5,0)$; (b) $(1,1,0)$ 


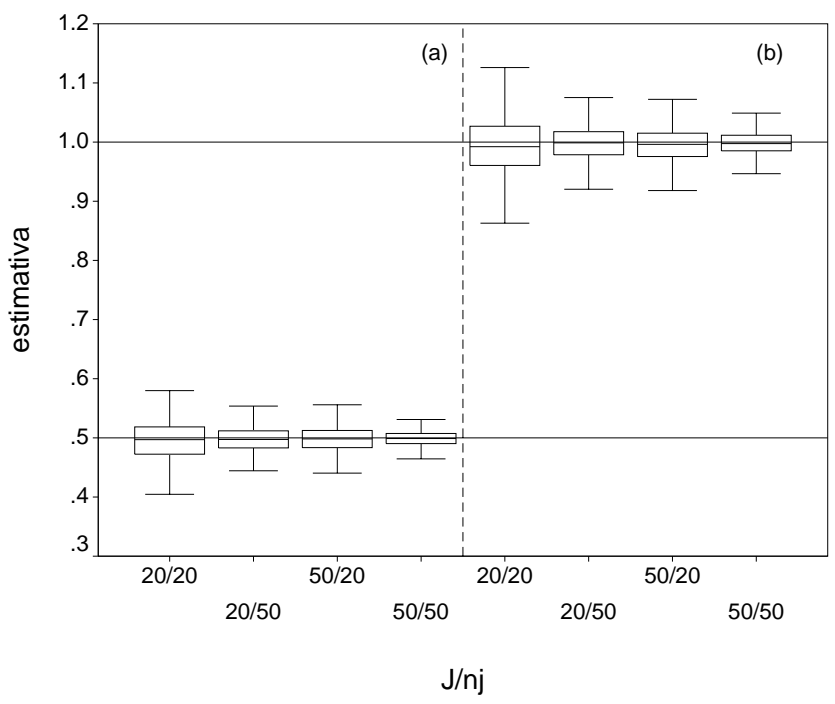

Figura C.13: Boxplots das estimativas de $\sigma$ quando $\left(\gamma_{00}, \tau_{0}, \sigma\right)$ é igual a (a) $(0,0,0.5) ; \quad$ (b) $(0,0,1)$

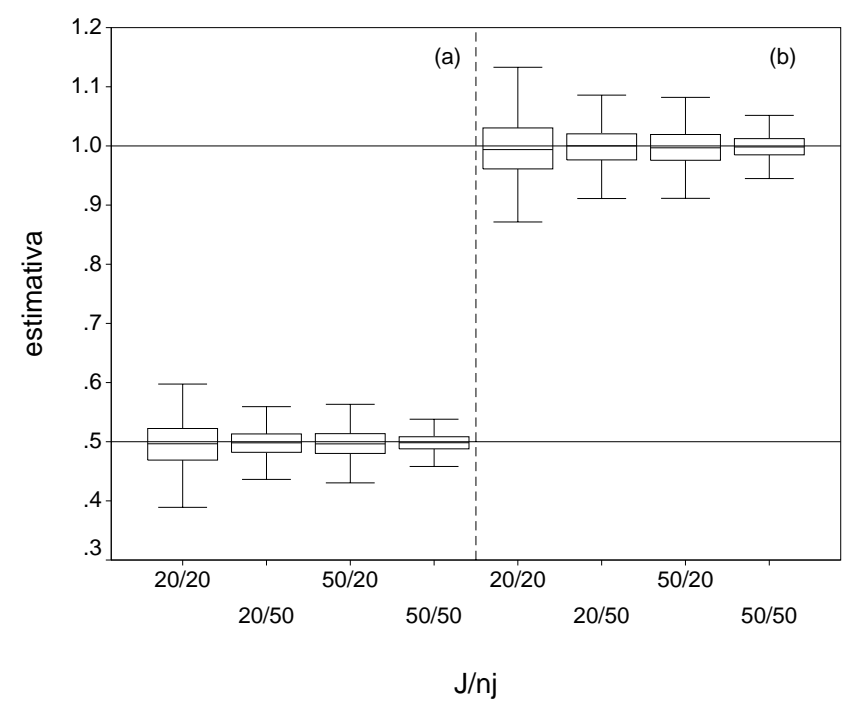

Figura C.14: Boxplots das estimativas de $\sigma$ quando $\left(\gamma_{00}, \tau_{0}, \sigma\right)$ é igual a (a) $(1,0,0.5) ; \quad$ (b) $(1,0,1)$ 


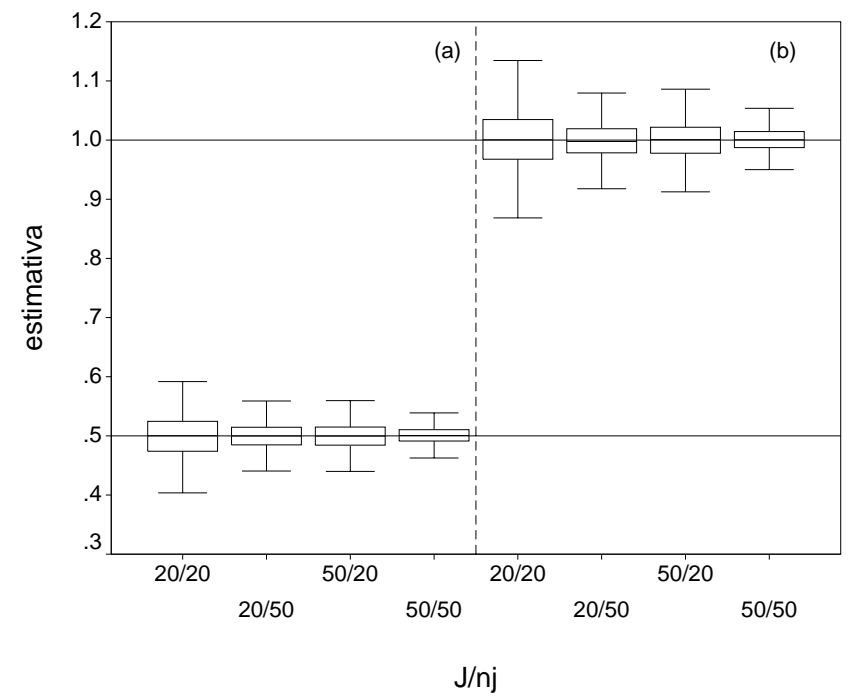

Figura C.15: Boxplots das estimativas de $\sigma$ quando $\left(\gamma_{00}, \tau_{0}, \sigma\right)$ é igual a (a) $(0,0.5,0.5) ; \quad$ (b) $(0,0.5,1)$

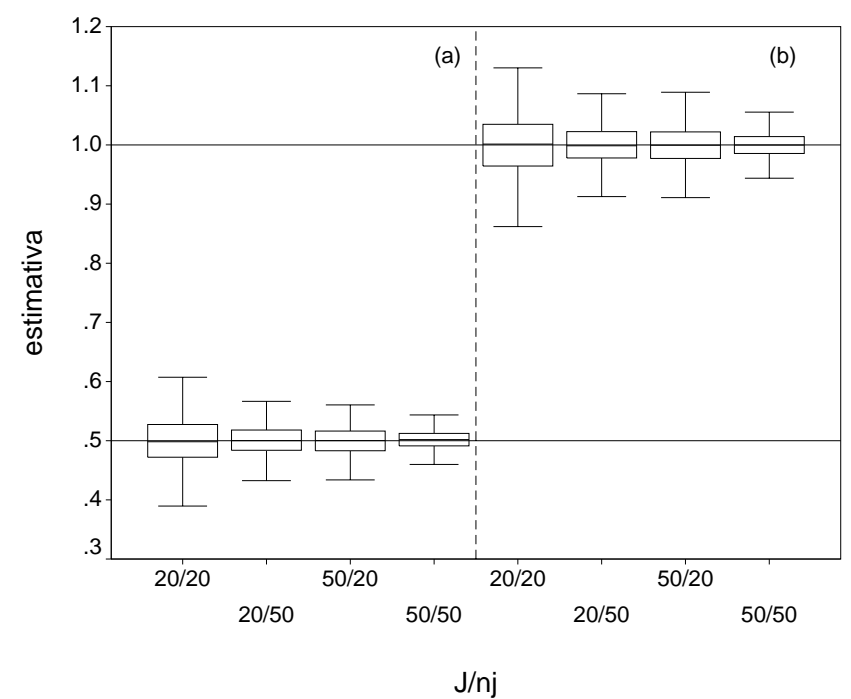

Figura C.16: Boxplots das estimativas de $\sigma$ quando $\left(\gamma_{00}, \tau_{0}, \sigma\right)$ é igual a (a) $(1,0.5,0.5) ; \quad$ (b) $(1,0.5,1)$ 


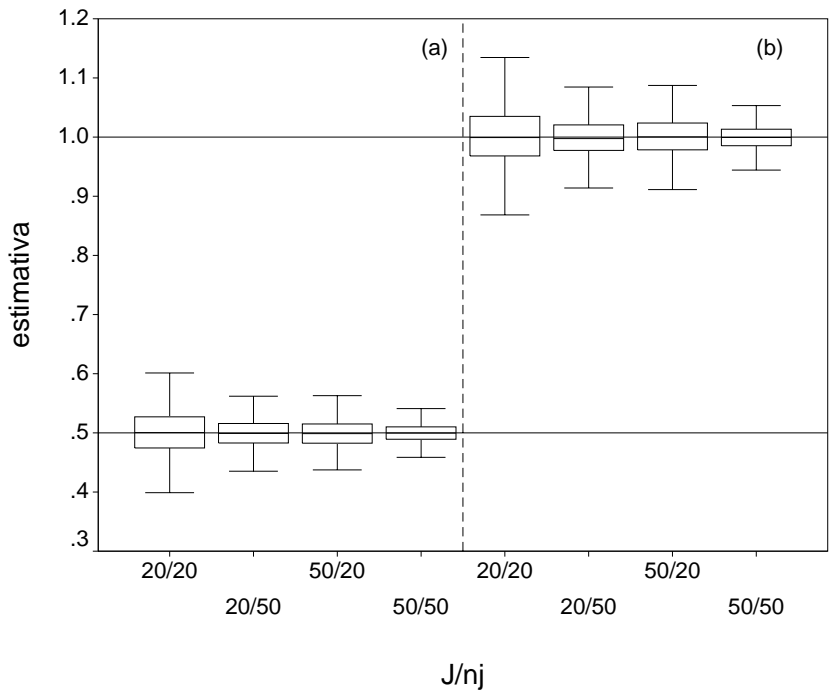

Figura C.17: Boxplots das estimativas de $\sigma$ quando $\left(\gamma_{00}, \tau_{0}, \sigma\right)$ é igual a (a) $(0,1,0.5) ; \quad$ (b) $(0,1,1)$

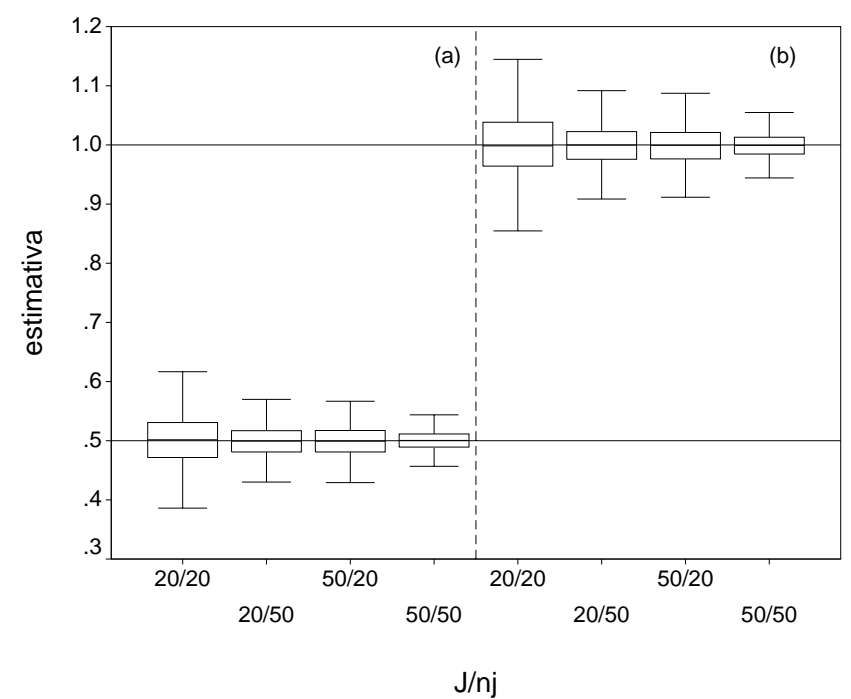

Figura C.18: Boxplots das estimativas de $\sigma$ quando $\left(\gamma_{00}, \tau_{0}, \sigma\right)$ é igual a (a) $(1,1,0.5) ; \quad$ (b) $(1,1,1)$ 


\section{Apêndice D}

\section{Gráficos referentes a $\gamma_{00}$}




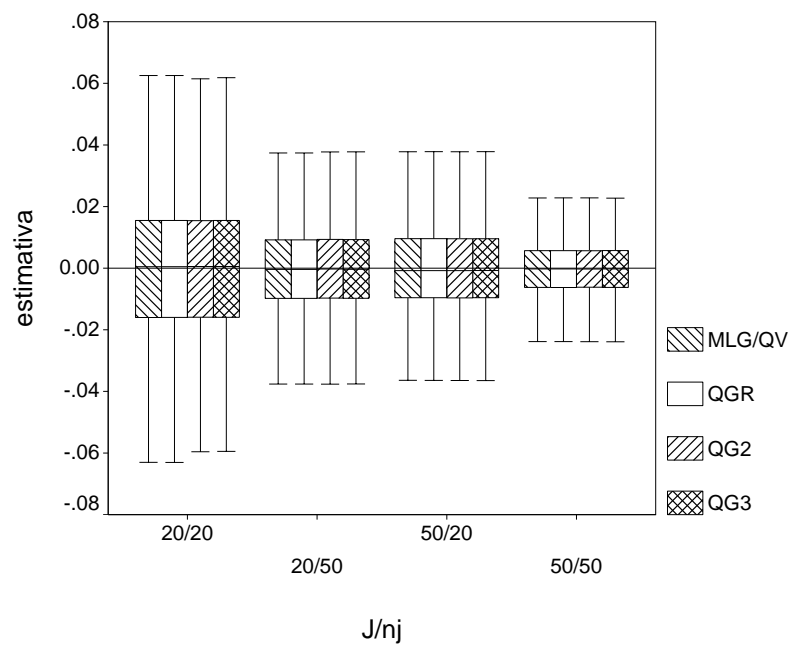

Figura D.1: Boxplots das estimativas de $\gamma_{00}$ quando $\left(\gamma_{00}, \tau_{0}, \sigma\right)=(0,0,0)$

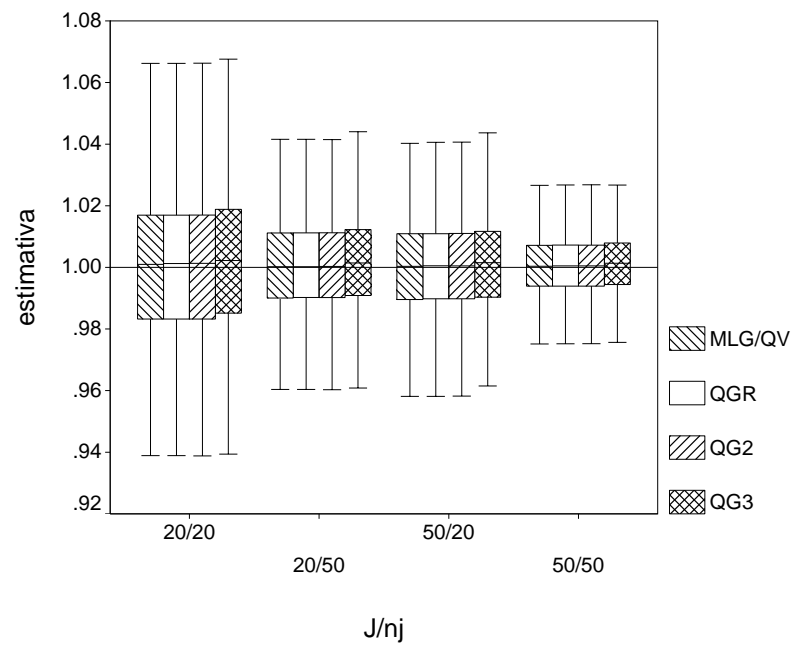

Figura D.2: Boxplots das estimativas de $\gamma_{00}$ quando $\left(\gamma_{00}, \tau_{0}, \sigma\right)=(1,0,0)$ 


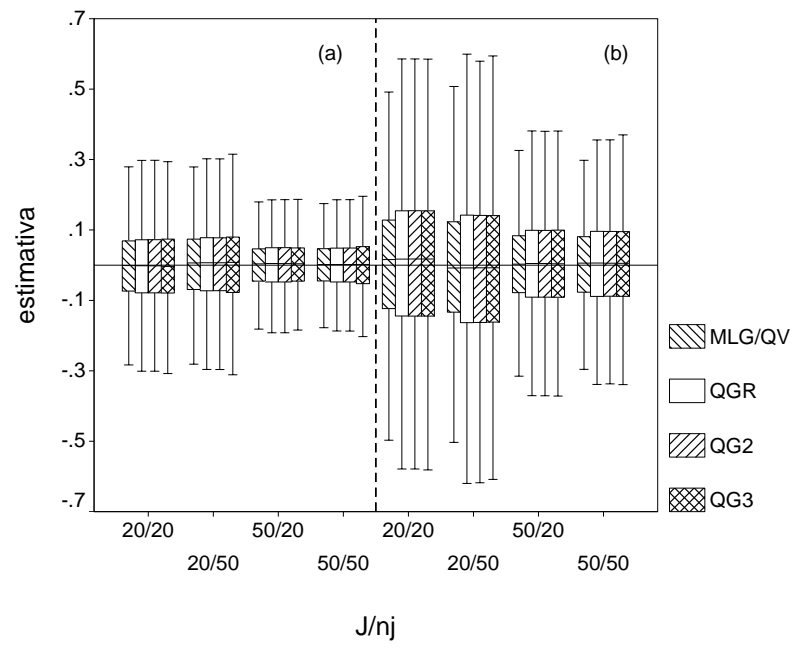

Figura D.3: Boxplots das estimativas de $\gamma_{00}$ quando $\left(\gamma_{00}, \tau_{0}, \sigma\right)$ é igual a (a) $(0,0.5,0) ; \quad$ (b) $(0,1,0)$

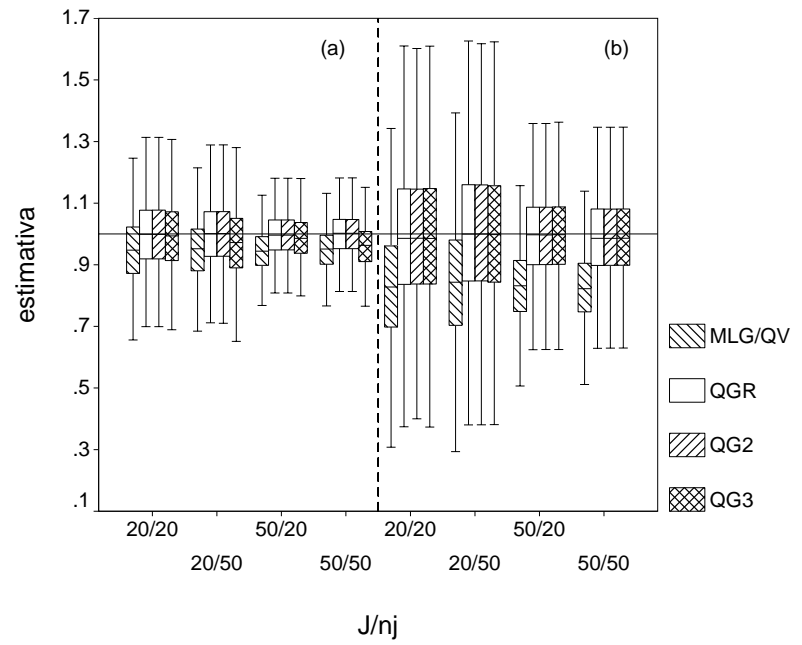

Figura D.4: Boxplots das estimativas de $\gamma_{00}$ quando $\left(\gamma_{00}, \tau_{0}, \sigma\right)$ é igual a (a) $(1,0.5,0)$; (b) $(1,1,0)$ 


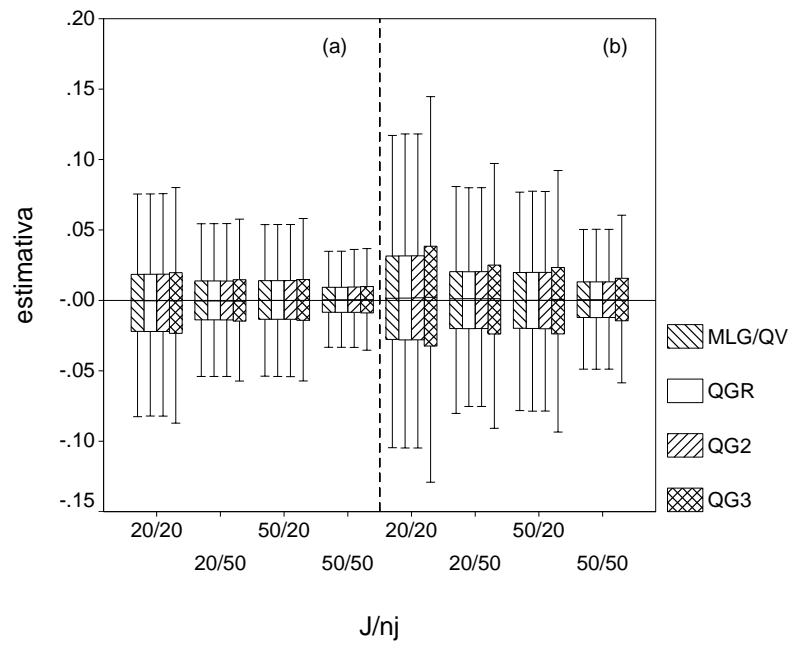

Figura D.5: Boxplots das estimativas de $\gamma_{00}$ quando $\left(\gamma_{00}, \tau_{0}, \sigma\right)$ é igual a (a) $(0,0,0.5) ; \quad$ (b) $(0,0,1)$

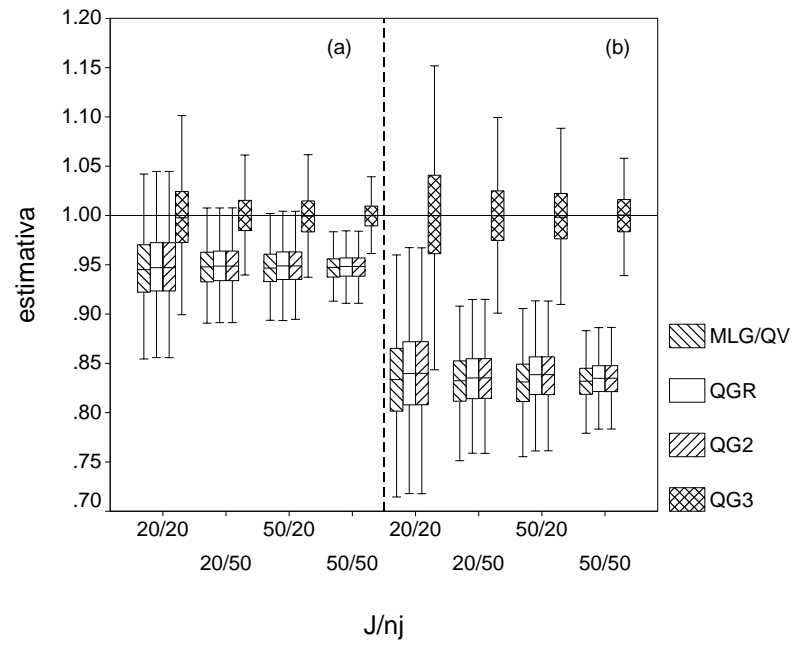

Figura D.6: Boxplots das estimativas de $\gamma_{00}$ quando $\left(\gamma_{00}, \tau_{0}, \sigma\right)$ é igual a
(a) $(1,0,0.5)$;
(b) $(1,0,1)$ 


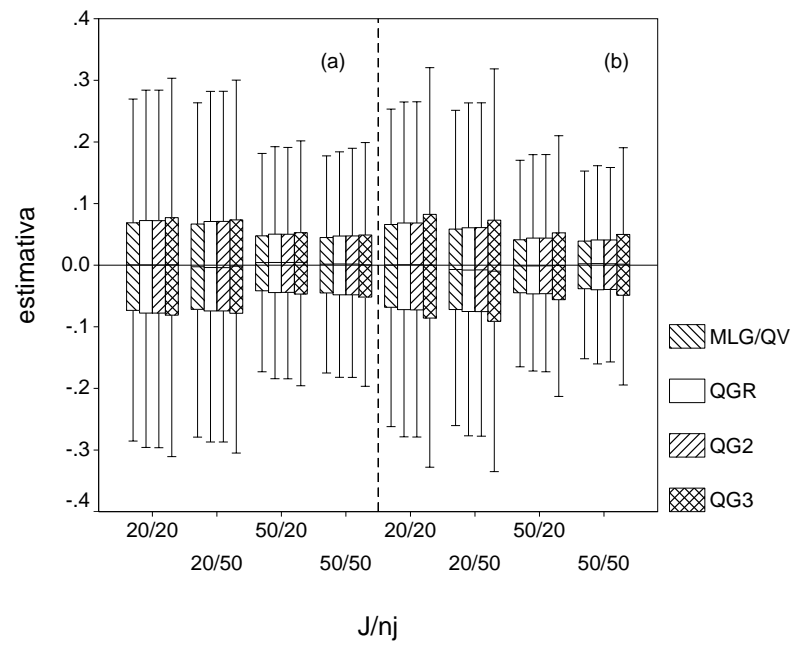

Figura D.7: Boxplots das estimativas de $\gamma_{00}$ quando $\left(\gamma_{00}, \tau_{0}, \sigma\right)$ é igual a (a) $(0,0.5,0.5) ; \quad$ (b) $(0,0.5,1)$

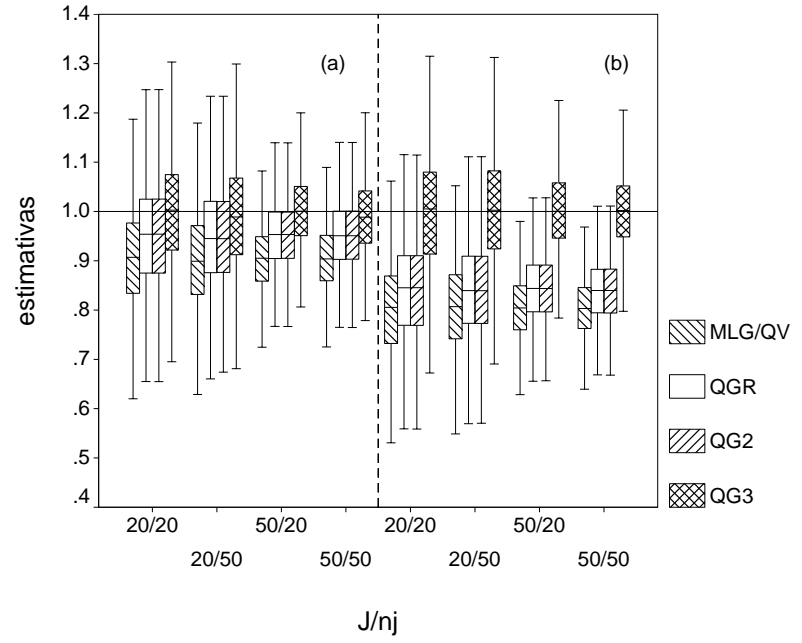

Figura D.8: Boxplots das estimativas de $\gamma_{00}$ quando $\left(\gamma_{00}, \tau_{0}, \sigma\right)$ é igual a (a) $(1,0.5,0.5)$; (b) $(1,0.5,1)$ 


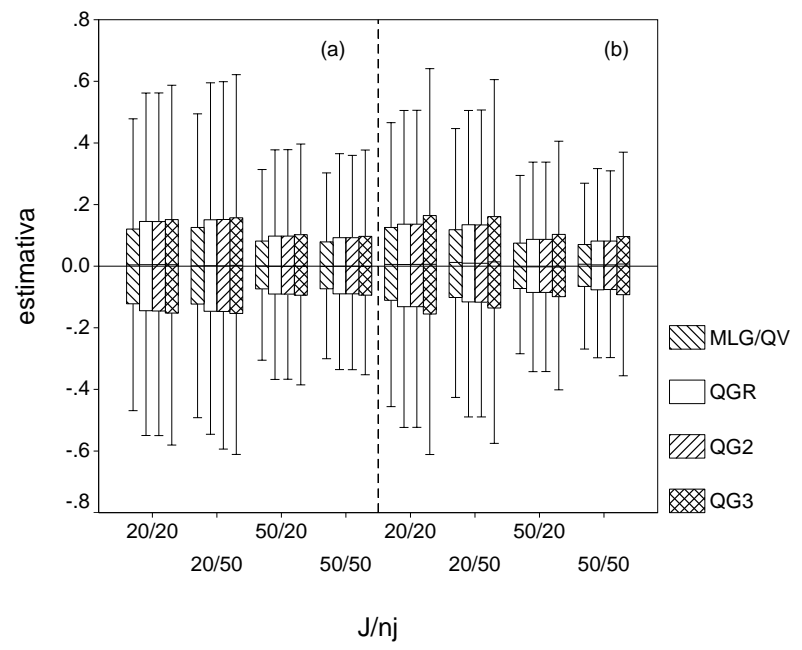

Figura D.9: Boxplots das estimativas de $\gamma_{00}$ quando $\left(\gamma_{00}, \tau_{0}, \sigma\right)$ é igual a (a) $(0,1,0.5) ; \quad$ (b) $(0,1,1)$

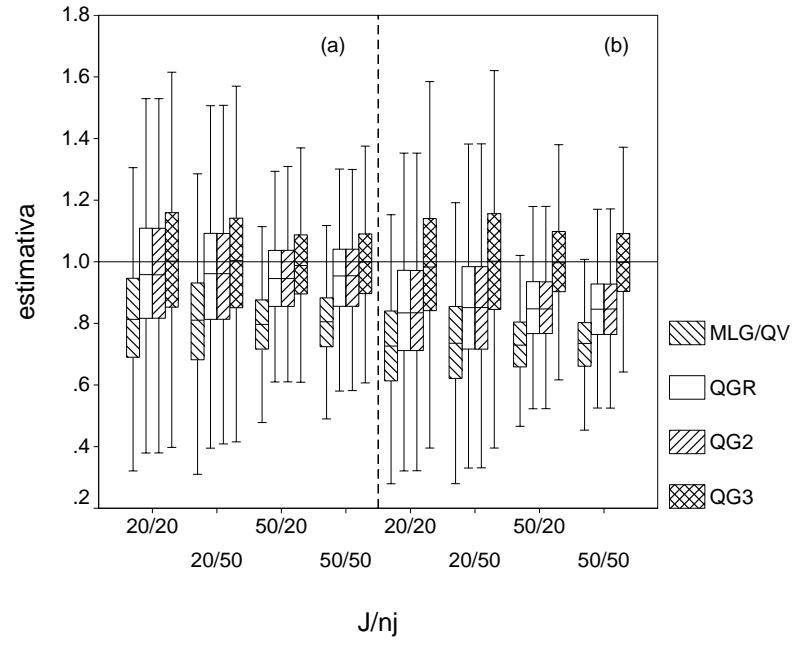

Figura D.10: Boxplots das estimativas de $\gamma_{00}$ quando $\left(\gamma_{00}, \tau_{0}, \sigma\right)$ é igual a (a) $(1,1,0.5)$; (b) $(1,1,1)$ 


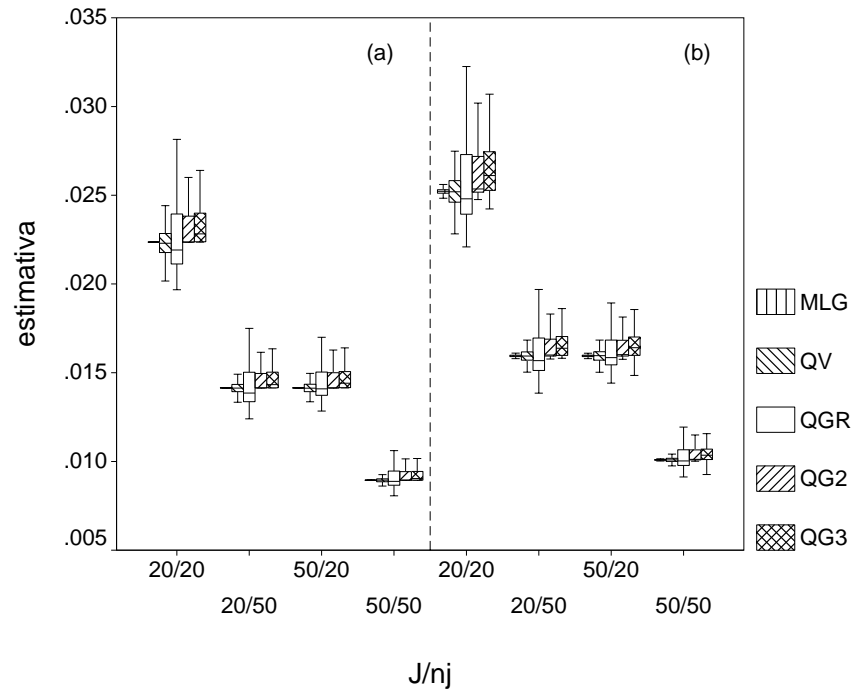

Figura D.11: Boxplots das estimativas do EP de $\hat{\gamma}_{00}$ quando $\left(\gamma_{00}, \tau_{0}, \sigma\right)$ é igual a (a) $(0,0,0) ; \quad$ (b) $(1,0,0)$ 


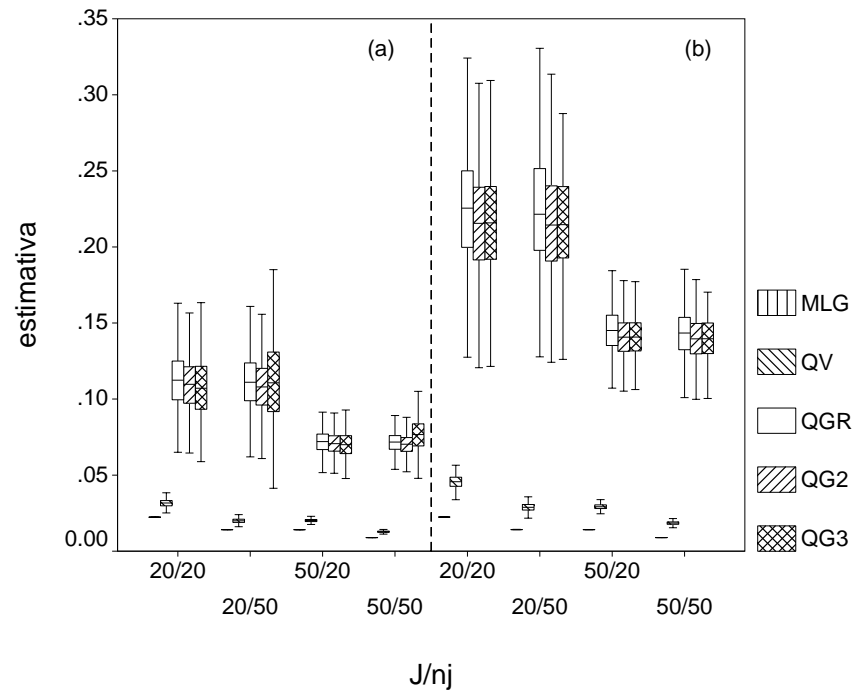

Figura D.12: Boxplots das estimativas do EP de $\hat{\gamma}_{00}$ quando $\left(\gamma_{00}, \tau_{0}, \sigma\right)$ é igual a (a) $(0,0.5,0) ; \quad$ (b) $(0,1,0)$

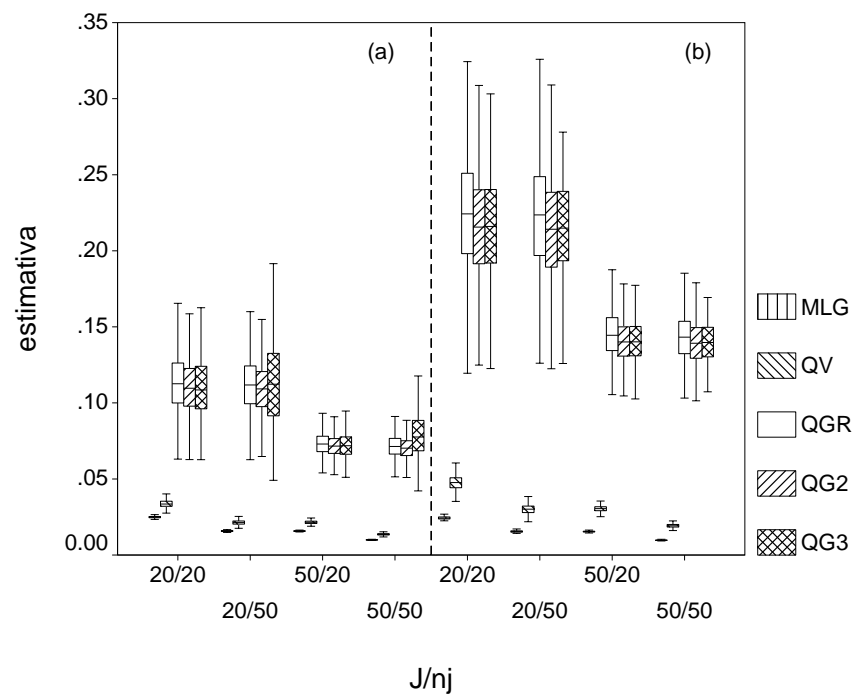

Figura D.13: Boxplots das estimativas do EP de $\hat{\gamma}_{00}$ quando $\left(\gamma_{00}, \tau_{0}, \sigma\right)$ é igual a (a) $(1,0.5,0)$; (b) $(1,1,0)$ 


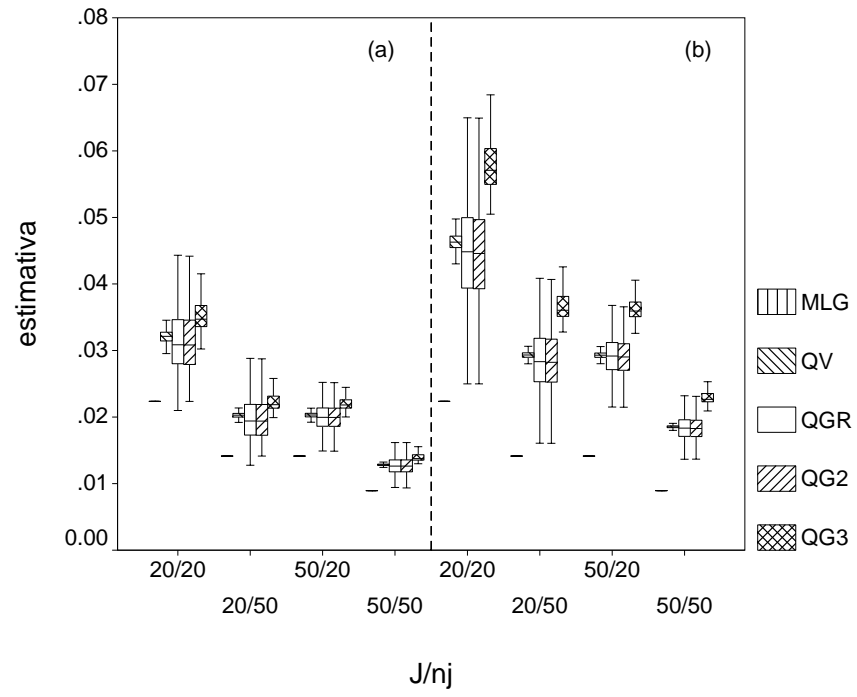

Figura D.14: Boxplots das estimativas do EP de $\hat{\gamma}_{00}$ quando $\left(\gamma_{00}, \tau_{0}, \sigma\right)$ é igual a (a) $(0,0,0.5) ; \quad$ (b) $(0,0,1)$

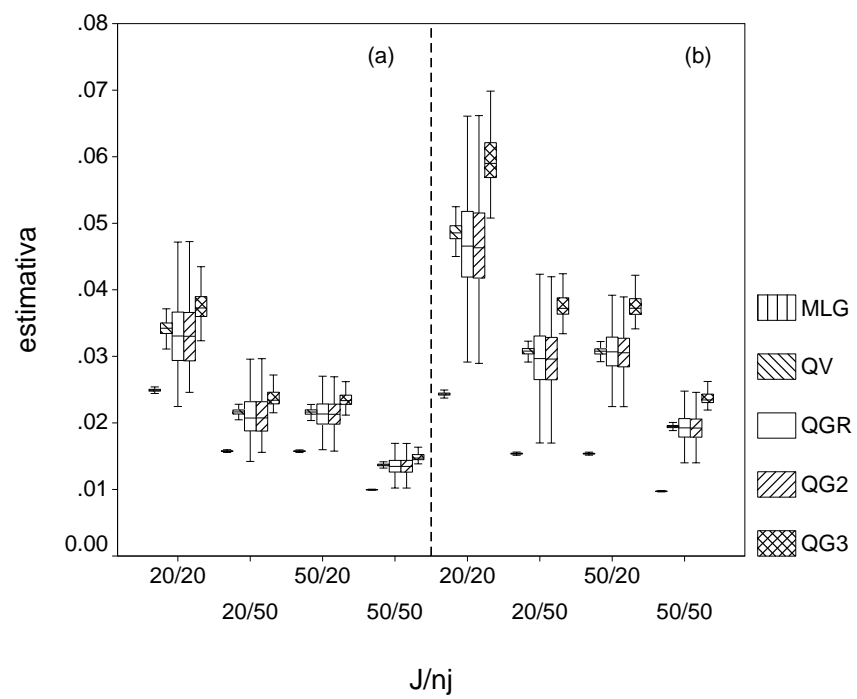

Figura D.15: Boxplots das estimativas do EP de $\hat{\gamma}_{00}$ quando $\left(\gamma_{00}, \tau_{0}, \sigma\right)$ é igual a (a) $(1,0,0.5) ; \quad$ (b) $(1,0,1)$ 


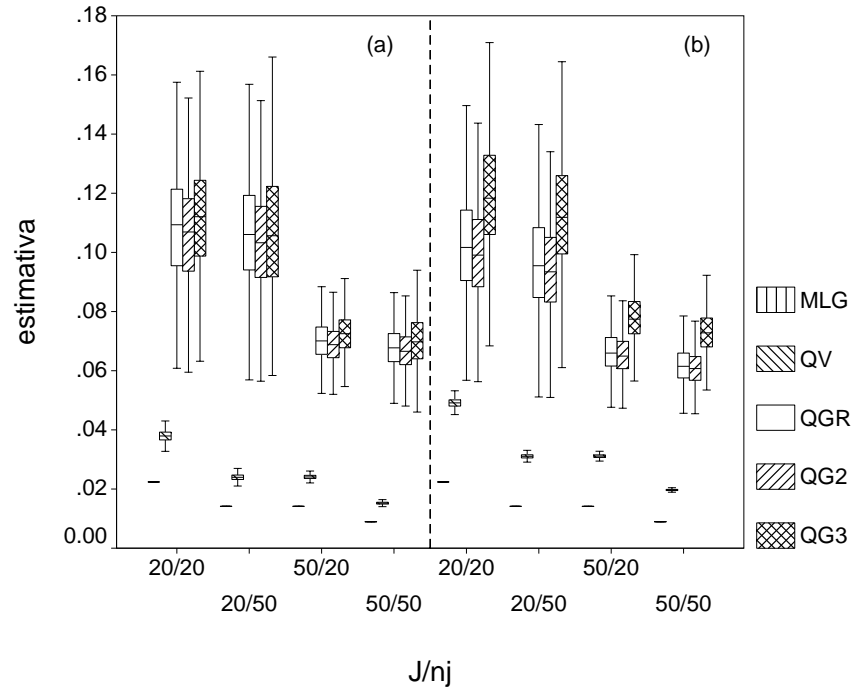

Figura D.16: Boxplots das estimativas do EP de $\hat{\gamma}_{00}$ quando $\left(\gamma_{00}, \tau_{0}, \sigma\right)$ é igual a (a) $(0,0.5,0.5) ; \quad$ (b) $(0,0.5,1)$

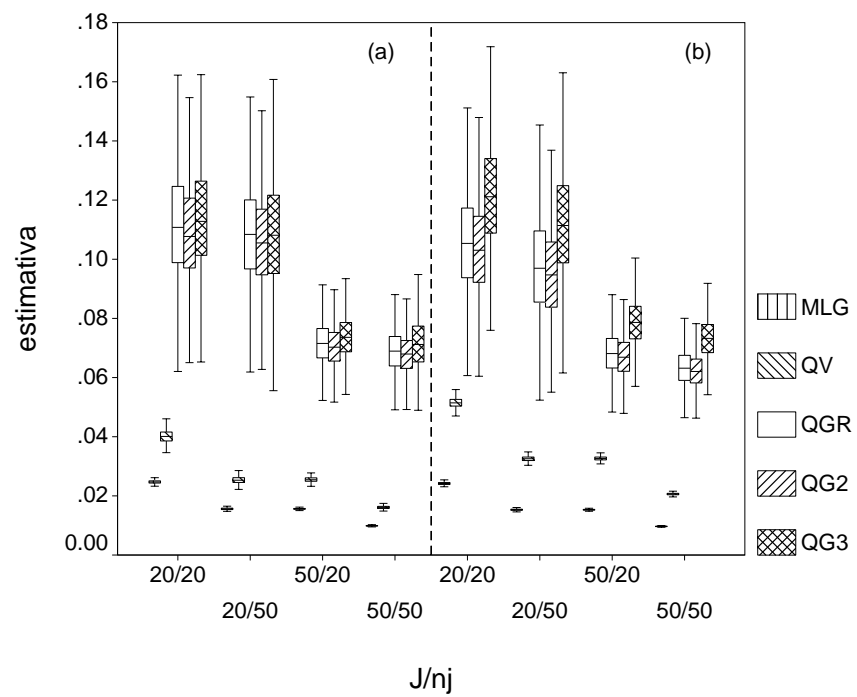

Figura D.17: Boxplots das estimativas do EP de $\hat{\gamma}_{00}$ quando $\left(\gamma_{00}, \tau_{0}, \sigma\right)$ é igual a (a) $(1,0.5,0.5) ; \quad$ (b) $(1,0.5,1)$ 


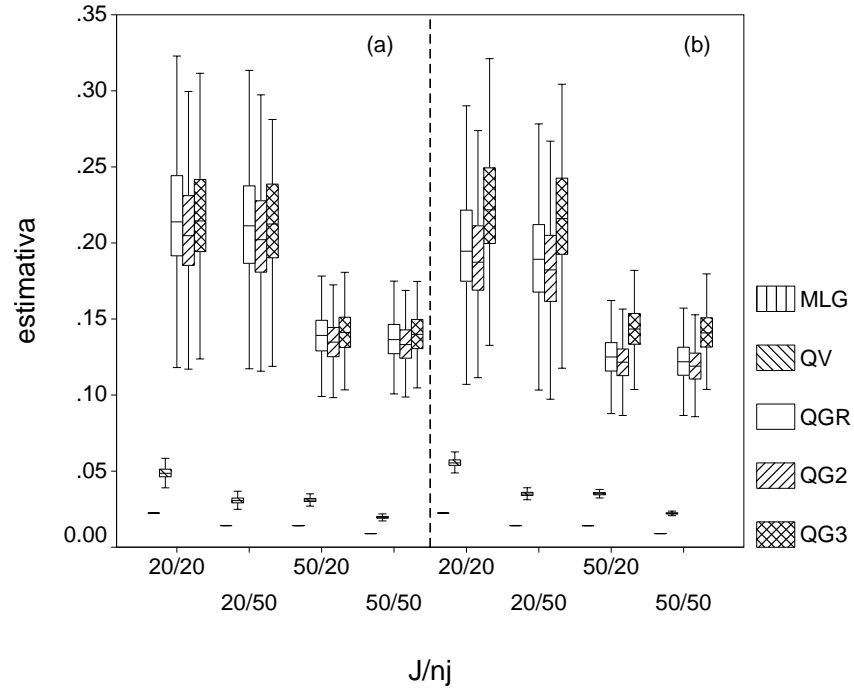

Figura D.18: Boxplots das estimativas do EP de $\hat{\gamma}_{00}$ quando $\left(\gamma_{00}, \tau_{0}, \sigma\right)$ é igual a (a) $(0,1,0.5) ; \quad$ (b) $(0,1,1)$

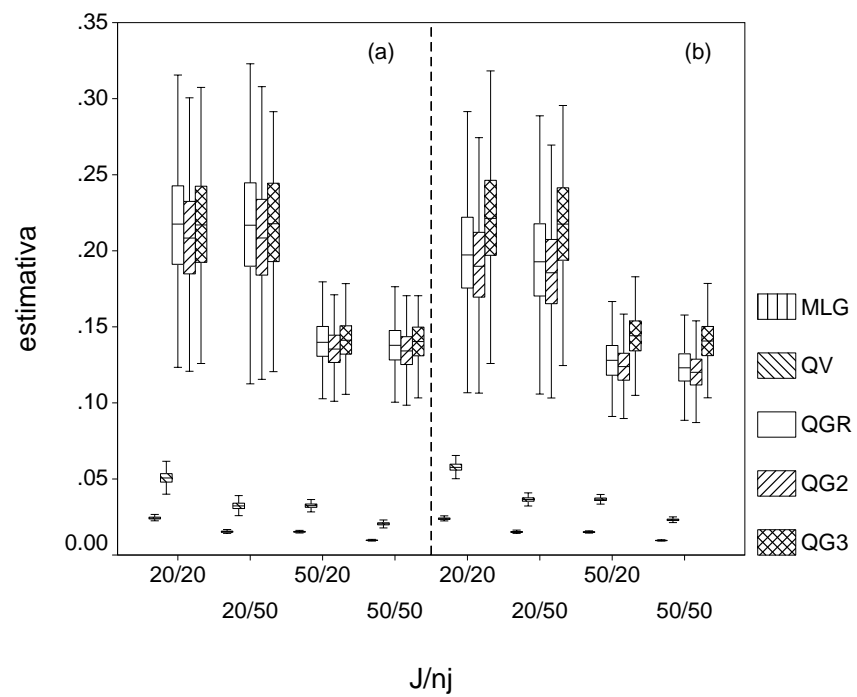

Figura D.19: Boxplots das estimativas do EP de $\hat{\gamma}_{00}$ quando $\left(\gamma_{00}, \tau_{0}, \sigma\right)$ é igual a (a) $(1,1,0.5)$; (b) $(1,1,1)$ 



\section{Referências Bibliográficas}

Anderson, D. A. e Aitkin, M. (1985). Variance component models with binary response: interviewer variability. Journal of the Royal Statistical Society, Series B: Statistical Methodology 47, 203210.

Breslow, N. E. e Clayton, D. G. (1993). Approximate inference in generalized linear mixed models. Journal of the American Statistical Association 88, 9-25.

Breslow, N. E. e Lin, X. (1995). Bias correction in generalised linear mixed models with a single component of dispersion. Biometrika 82, 81-91.

Davidian, M. e Giltinan, D. (1995). Nonlinear models for repeated measurement data. London: Chapman \& Hall.

Diggle, P., Heagerty, P., Liang, K.-Y. e Zeger, S. (2002). Analysis of longitudinal data. $2^{\mathrm{a}}$ ed. Oxford: Oxford University Press.

Efron, B. (1986). Double exponential families and their use in generalized linear regression. Journal of the American Statistical Association 81, 709-721.

Fahrmeir, L. e Tutz, G. (2001). Multivariate statistical modelling based on generalized linear models. $2^{\mathrm{a}}$ ed. New York: Springer-Verlag.

Goldstein, H. (1991). Nonlinear multilevel models, with an application to discrete response data. Biometrika 78, 45-51.

Goldstein, H. (2003). Multilevel statistical models. $3^{\mathrm{a}}$ ed. New York : Edward Arnold. 
Hedeker, D. e Gibbons, R. D. (1994). A random-effects ordinal regression model for multilevel analysis. Biometrics 50, 933-944.

Hinde, J. e Demétrio, C. (1998). Overdispersion: models and estimation. São Paulo: Associação Brasileira de Estatística.

Jacob, M. (2000). Extra-binomial variation in logistic multilevel models - a simulation. Multilevel Modelling Newsletter 12(1), 8-14.

Lee, Y. e Nelder, J. A. (1996). Hierarquical generalized linear models. Journal of the Royal Statistical Society, Series B 58(4), 619-678.

Lee, Y. e Nelder, J. A. (2006). Double hierarchical generalized linear models. Applied Statistics 55, 139-185.

Lee, Y., Nelder, J. A. e Pawitan, Y. (2006). Generalized linear models with random effects. Unified analysis via h-likelihood. New York: Chapman \& Hall.

Lin, X. e Breslow, N. E. (1996). Bias correction in generalised linear mixed models with multiple components of dispersion. Journal of the American Statistical Association 91, 1007-1016.

Liu, Q. e Pierce, D. A. (1994). A note on Gauss-Hermite quadrature. Biometrika 81(3), 624-629.

McCullagh, P. e Nelder, J. A. (1989). Generalized linear models. $2^{\text {a }}$ ed. New York: Chapman \& Hall.

Molenberghs, G. e Verbeke, G. (2005). Models for discrete longitudinal data. New York: Springer Science.

Natis, L. (2000). Modelos lineares hierárquicos. Dissertação de mestrado. Instituto de Matemática e Estatística, Universidade de São Paulo.

Nelder, J. A. e Mead, R. (1965). A simplex algorithm for function minimization. Computer Journal 7, 308-313.

Nobre, J. S. (2004). Métodos de diagnóstico para modelos lineares mistos. Dissertação de mestrado. Instituto de Matemática e Estatística, Universidade de São Paulo.

Nocedal, J. e Wright, S. J. (1999). Numerical optimization. New York: Springer-Verlag. 
Paula, G. (2004). Modelos de regressão com apoio computacional. São Paulo: Instituto de Matemática e Estatística, Universidade de São Paulo.

Pinheiro, J. C. e Bates, D. M. (1995). Approximations to the log-likelihood function in the nonlinear mixed-effects model. Journal of Computational and Graphical Statistics 4, 12-35.

Poli, E. C. (2000). Estudo longitudinal em matemática: possibilidades e leitura de uma realidade do ensino fundamental. Tese de doutorado. Campinas: Faculdade de Educação, Universidade Estadual de Campinas.

Raudenbush, S. W. e Bryk, A. S. (2002). Hierarquical linear models: applications and data analysis methods. $2^{\text {a }}$ ed. Thousand Oaks: Sage Publications.

Raudenbush, S. W., Yang, M. e Yosef, M. (2000). Maximum likelihood for hierarchical models via high-order, multivariate Laplace aproximation. Journal of Computational and Graphical Statistics 9(1), 141-157.

Wolfinger, R. D. e O'Connell, M. (1993). Generalized linear mixed models: a pseudo-likelihood approach. Journal of Statistical Computation and Simulation 48, 233-243.

Zeger, S. L. e Karim, M. R. (1991). Generalized linear models with random effects; a Gibbs sampling approach. Journal of the American Statistical Association 86, 79-86. 
